\title{
Towards tailored rehabilitation programs after lower extremity fractures
}

Citation for published version (APA):

Raaben, M. (2020). Towards tailored rehabilitation programs after lower extremity fractures. [Doctoral Thesis, Maastricht University]. Maastricht University. https://doi.org/10.26481/dis.20200501 mr

Document status and date:

Published: 01/01/2020

DOI:

10.26481/dis.20200501mr

Document Version:

Publisher's PDF, also known as Version of record

\section{Please check the document version of this publication:}

- A submitted manuscript is the version of the article upon submission and before peer-review. There can be important differences between the submitted version and the official published version of record.

People interested in the research are advised to contact the author for the final version of the publication, or visit the DOI to the publisher's website.

- The final author version and the galley proof are versions of the publication after peer review.

- The final published version features the final layout of the paper including the volume, issue and page numbers.

Link to publication

\footnotetext{
General rights rights.

- You may freely distribute the URL identifying the publication in the public portal. please follow below link for the End User Agreement:

www.umlib.nl/taverne-license

Take down policy

If you believe that this document breaches copyright please contact us at:

repository@maastrichtuniversity.nl

providing details and we will investigate your claim.
}

Copyright and moral rights for the publications made accessible in the public portal are retained by the authors and/or other copyright owners and it is a condition of accessing publications that users recognise and abide by the legal requirements associated with these

- Users may download and print one copy of any publication from the public portal for the purpose of private study or research.

- You may not further distribute the material or use it for any profit-making activity or commercial gain

If the publication is distributed under the terms of Article $25 \mathrm{fa}$ of the Dutch Copyright Act, indicated by the "Taverne" license above, 



\section{Towards tailored rehabilitation programs after lower extremity fractures}

Marco Raaben 


\section{Colofon}

(C) Copyright Marco Raaben, 2020

All rights reserved. No part of this book may be reproduced or transmitted in any form or by any means, without prior permission in writing by the author, or when appropriate, by the publishers of the publications.

ISBN: 978-94-028-1997-7

The work described in this thesis has received funding from the Eurostars- 2 joint programme with co-funding from the European Union Horizon 2020 research and innovation programme.

Financial support by the Maastricht University for the publication of this thesis is gratefully acknowledged. 


\title{
Towards tailored rehabilitation programs after lower extremity fractures
}

\author{
PROEFSCHRIFT
}

ter verkrijging van de graad van doctor aan de Universiteit Maastricht, op gezag van de Rector Magnificus, prof. dr. Rianne M. Letschert volgens het besluit van het College van Decanen, in het openbaar te verdedigen

op vrijdag 1 mei 2020 om 12.00 uur

door

Marco Raaben

geboren op 30 juli 1988 te Neede 


\section{Promotor}

Prof. dr. M. Poeze

\section{Copromotor}

Dr. T.J. Blokhuis

Leden van beoordelingscommissie

Prof. dr. L.W. van Rhijn (voorzitter)

Prof. dr. M.J.R. Edwards (Radboud Universiteit)

Prof. dr. ir. H.J. Hermens (Universiteit Twente)

Prof. dr. A.F. Lenssen

Prof. dr. G.M. Rommers 



\section{Table of Contents}

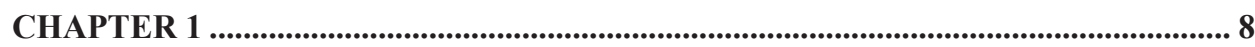

General introduction and outline

\section{PART 1 - THE IMPORTANCE OF EARLY WEIGHT BEARING}

\section{CHAPTER 2}

Delay in weight bearing in surgically treated tibial shaft fractures is associated with impaired healing: a cohort analysis of 166 tibial fractures

\section{CHAPTER 3} 38

Technical Aspects and Validation of a New Biofeedback System for Measuring Lower Limb Loading in the Dynamic Situation

\section{PART 2 - REAL-TIME BIOFEEDBACK}

\section{CHAPTER 4}

Real-time visual biofeedback during weight bearing improves therapy compliance in patients following lower extremity fractures

\section{CHAPTER 5}

Real-time visual biofeedback to improve therapy compliance after total hip arthroplasty: A pilot randomized controlled trial

\section{PART 3 - BIOFEEDBACK IN THE ELDERLY POPULATION}

\section{CHAPTER 6} 90

Innovative Measurement Of Rehabilitation Progress In Elderly With A Hip Fracture: A New Endpoint 
CHAPTER 7

COMplex Fracture Orthopedic Rehabilitation (COMFORT) - Real-time visual biofeedback on weight bearing versus standard training methods in the treatment of proximal femur fractures in the elderly: study protocol for a multicenter randomized controlled trial

\section{CHAPTER 8}

Real-time biofeedback improves rehabilitation after hip fractures in elderly: an international, multicenter randomised controlled trial (COMFORT)

\section{CHAPTER 9}

General discussion and summary

CHAPTER 10 146

Nederlandse samenvatting en toekomstperspectieven (Dutch summary)

\section{ADDENDUM}

Valorisation

List of publications

International abstracts and poster sessions

Acknowledgements

Curriculum Vitae 
CHAPTER
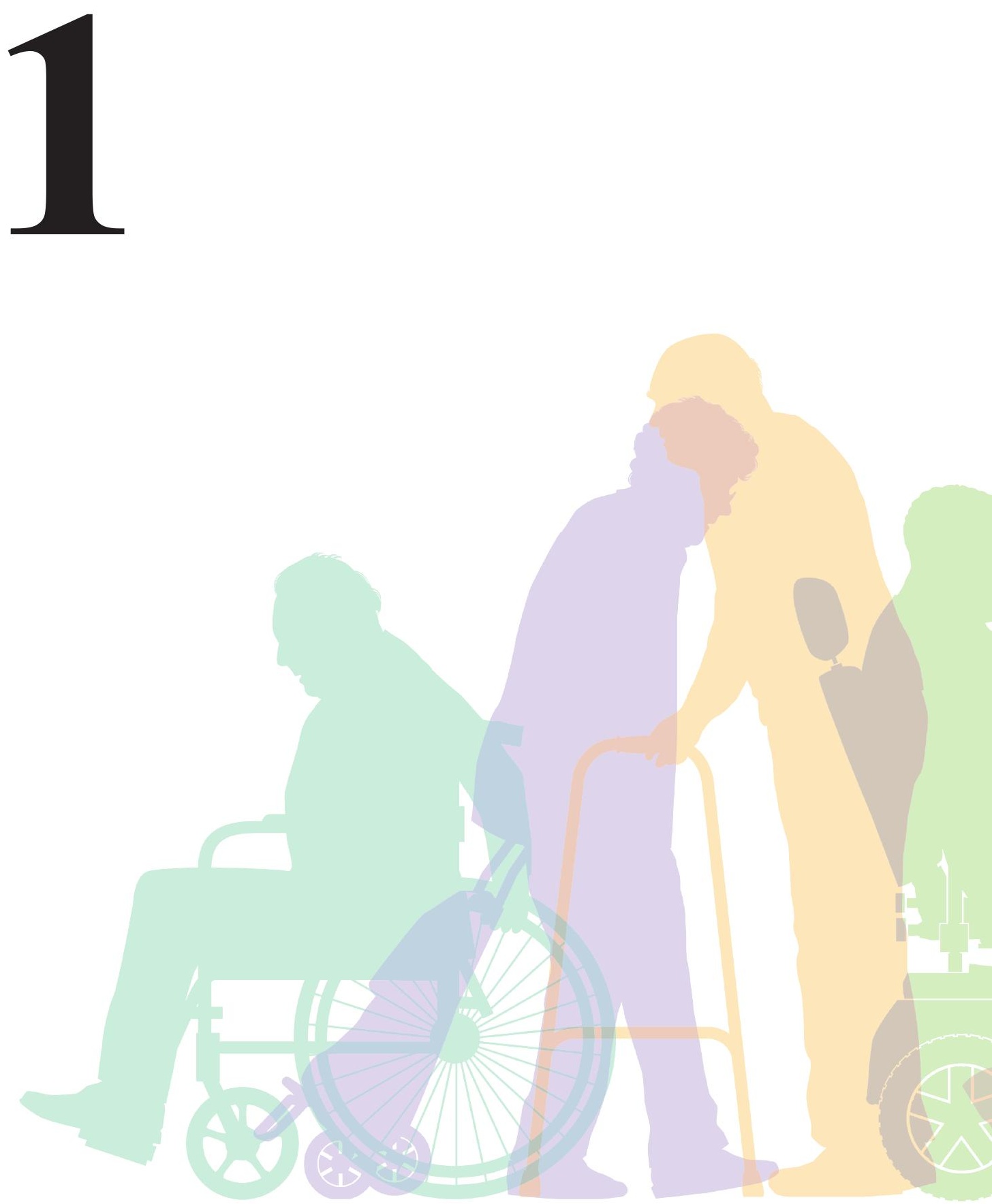
General introduction and outline 


\section{Lower extremity fractures}

Bone gives structure to our body and provides support and protection to the soft tissues and internal organs. It is a living tissue that is continuously remodelled by a specialised group of cells called basic multicellular units, which consist of bone resorbing osteoclasts and bone forming osteoblasts. The importance of mechanical loading in this bone remodelling process was already described in the $19^{\text {th }}$ century by the German surgeon Julius Wolff [1] Wolff's law describes that bone will adapt itself to the load under which it is exposed, in order to become stronger and be able to resist the necessary amount of loading. Later, the exact mechanism behind bone remodelling was revealed, and it has been shown that mechanical load induces a fluid flow in the canalicular network. This fluid flow acts as a physical mediator for mechanosensing by osteocytes and is recognized by extracellular matrix transmembrane receptors such as integrins and CD44 receptors. The osteocytes respond to the mechanical stimuli via intracellular pathways and start with the production of signalling molecules, such as prostaglandins and nitric oxide, that modulate the activity of osteoblasts and osteoclasts. Through this mechanism, the mechanical stimuli induced by loading are converted into cellular signals that initiate bone remodelling. This process is described in literature as the mechanotransduction in bone [2]. Mechanotransduction is continuously present and enables the bone to resist the mechanical impacts caused by daily activities.

Although bone has the ability to adapt to various mechanical needs, excessive mechanical impacts could cause bone fractures. Fortunately, fracture healing is a unique process in the human being that results in the reconstruction of the fractured bone into something very similar to its original form [3]. Five factors contribute to this process, defined as the diamond concept: 1) osteogenic cells, 2) osteoconductive scaffolds, 3) growth factors, 4) vascularity and 5) the mechanical environment [4]. The first four factors reflect the biological environment of the patient and are thus mainly patient related. The mechanical environment, however, can be influenced by the treating physician by choosing the correct treatment options and rehabilitation program. First, a meticulous surgical approach is necessary to create the optimal mechanical environment. Although surgical techniques have evolved over the years, it is still difficult to determine the optimal treatment strategy for every specific fracture. Recent large multicenter randomised controlled trials (RCTs) such as INSITE (Intramedullary Nail Versus Sliding Hip Screw Inter-Trochanteric Evaluation) 
and HEALTH (Hip Fracture Evaluation with ALternatives of Total Hip Arthroplasty versus Hemiarthroplasty) illustrate the current struggle of surgeons in choosing the optimal implant $[5,6]$. A diversity of surgical techniques is nowadays available to treat all types of fractures in various types of patients. The surgeon has to determine the correct surgical technique for each individual case in order to create the optimal mechanical environment at the fracture site.

Then, secondly and equally important, after surgical fixation the optimal rehabilitation program should be determined for each individual patient. Rehabilitation is primarily important for the fracture healing outcome, but also for patients to regain their gait, improve muscle mass and general coordination [7]. In this light, it is remarkable that there is an absence of large, high quality RCTs, such as INSITE and HEALTH, to investigate the optimal rehabilitation program for each patient. In contrast, the rehabilitation is mainly experience based instead of evidence based. It depends on the treating physician which rehabilitation program is chosen and often varies significantly between no weight bearing, touch-down weight bearing, partial weight bearing and immediate full weight bearing. The treating physician is thereby balancing between protecting the mechanical construct at the fracture site or stimulating bone healing by mechanical load [8]. High mechanical loads, for example, could cause failure of the mechanical construct and/or failure of the fracture fixation [9]. On the other hand, early weight bearing leads to mechanotransduction at the fracture site and thereby bone remodelling as previously described by Wolff's law $[1,2]$. Other beneficial aspects of early weight bearing are improved coordination and muscle mass [7]. With these arguments in mind, it is remarkable that there is still no consensus about the correct weight bearing regime after lower extremity fractures. Moreover, there is an absence of worldwide guidelines on rehabilitation after lower extremity fractures, while high quality rehabilitation is important to improve fracture healing outcome.

\section{Rehabilitation after lower extremity fractures}

The absence of worldwide guidelines can be explained by the limited availability of necessary rehabilitation tools. In daily practice, bathrooms scales are often used to train weight bearing and provide the correct feeling of weight bearing in the static situation. However, the information is lost in the dynamic situation and also the treating healthcare professional has no information about the amount of applied weight during gait. This is the 
main reason that therapy compliance is low and patients often under- or overload their fractured lower extremity, with impaired bone healing or fixation failure as a result [9-12].

Biofeedback systems have shown their potential to improve therapy compliance as these systems are able to provide insight in weight bearing in the dynamic situation. The first reported biofeedback system was already in 1974 developed by Endicott et al [13]. The development of biofeedback systems continued and many improvements have been made over the years [14]. Although many beneficial effects of biofeedback systems were described, also many drawbacks remained. For example, most biofeedback systems are restricted to laboratory use. Systems that are able to measure gait in the ambulatory, clinical setting are either unreliable, or unable to provide real-time biofeedback. The demand for a proper biofeedback system able to reliably and continuously measure gait in the ambulatory, clinical setting was still present. The first aim of this $\mathrm{PhD}$ thesis was therefore to develop a new and innovative biofeedback system to overcome previous limitations. This resulted in the development of SensiStep.

\section{Hip fractures in elderly}

The SensiStep was used as innovative rehabilitation tool to improve fracture healing outcome in a frail population: elderly with a hip fracture. This specific population is important as the incidence of hip fractures is rising from 1.31 million in 1990 to a predicted incidence of 6.26 million in 2050 [15]. One explanation is the increasing worldwide life expectancy and thereby the survival of frail and elderly people with a high risk of falls [16]. Recent reports from the UK National Hip Fracture database (NHFD) showed that hip fractures typically occur in the elderly population (i.e. 91.6\%). Most of the patients are females $(72 \%)$ and the hip fractures are the result from a low energy trauma [17]. Besides the high impact on society, hip fractures in elderly also have a high morbidity and mortality. Approximately 30\% will not survive the first year following a hip fracture [18]. Another 30\% will survive, but with an ongoing burden significantly affecting quality of life [19]. The remaining group of patients will recover to their pre-fracture mobility.

Especially the second group of patients could significantly benefit from high quality rehabilitation programs. These patients often rehabilitate in geriatric rehabilitation clinics to improve healing outcome. Physical therapists intensively train patients to improve their gait in daily, targeted training sessions [20]. However, one of the problems in current training sessions, both experienced by the patient and physical therapists, is the limited availability 
of adequate feedback methods during gait. SensiStep has the potential to solve this problem as the system is able to provide real-time biofeedback during gait to the patient and physical therapist. This could improve therapy compliance and thereby fracture healing outcome and mobility after hip fractures in elderly. Therefore, the second aim of this $\mathrm{PhD}$ thesis was to measure gait and analyse the effect of real-time biofeedback on gait in the elderly population following a hip fracture. The results could be used to develop high quality rehabilitation programs and improve fracture healing outcome.

\section{General aim of this thesis}

The aim of this thesis was to develop an accurate, reliable and ambulatory biofeedback system able to provide real-time feedback in the clinical setting during the rehabilitation after lower extremity fractures. The focus was to use this innovative biofeedback system for the development of high quality rehabilitation programs after hip fractures in elderly. This could lead to improved fracture healing outcome, thereby improved quality of life for patients and lower economic costs for society.

\section{Outline of this thesis}

This thesis is divided into three parts. The outline of this thesis is first to describe the importance of early weight bearing (part 1, chapters 2-3) and then evaluate the effect of real-time biofeedback on weight bearing during rehabilitation after lower extremity fractures (part 2, chapters 4-5), with focus on hip fractures in elderly (part 3, chapters 6-8).

Part 1 gives a general introduction about the importance of weight bearing on fracture healing and the development of a biofeedback system to improve therapy compliance. Chapter 2 describes a retrospective study that showed that any delay in initial weight bearing is independently associated with impaired fracture healing. Correct and early weight bearing is important and therefore we developed a new biofeedback system using state-of-the-art sensor technologies to improve therapy compliance after lower extremity fractures. Technical details and the validation of this system can be found in chapter 3 .

Part 2 evaluates the effect of ambulatory, real-time biofeedback on weight bearing in the clinical setting. The beneficial effects and potential use of biofeedback systems during rehabilitation after lower extremity fractures are described in chapter 4. Significant improvements in weight bearing were found in patients with touch-down weight bearing as well as in patients with full weight bearing. Also, significant improvements in weight 
bearing were found in elderly patients following a total hip arthroplasty as described in chapter 5.

Part 3 investigates the use of biofeedback systems in the frail, elderly population. Chapter 6 gives insight in the normal gait patterns and rehabilitation progress after hip fractures in elderly. Important gait parameters were described, as well as the new objective endpoint of rehabilitation. The question remains if real-time biofeedback about these parameters leads to improved gait patterns and thereby improved fracture healing outcome. A study proposal to answer this question is described in chapter 7. The results of this study can be found in chapter 8. Real-time biofeedback resulted in a significant improvement in weight bearing compared to standard training methods. These results are summarised in chapter 9 and implied that SensiStep should be used in future rehabilitation programs to improve the rehabilitation after hip fractures in elderly. 


\section{References}

[1] J. Wolff, Das Gesetz der Transformation der Knochen, 1892.

[2] J. Klein-Nulend, R.G. Bacabac, M.G. Mullender, Mechanobiology of bone tissue, Pathol. Biol. 53 (2005) 576580. doi:10.1016/j.patbio.2004.12.005.

[3] R. Marsell, T.A. Einhorn, The biology of fracture healing, Injury. 42 (2011) 551-555. doi:10.1016/j.injury.2011.03.031.

[4] P. V. Giannoudis, T.A. Einhorn, G. Schmidmaier, D. Marsh, The diamond concept - open questions, Injury. 39 (2008). doi:10.1016/S0020-1383(08)70010-X.

[5] M.J. Parker, Sliding hip screw versus intramedullary nail for trochanteric hip fractures; a randomised trial of 1000 patients with presentation of results related to fracture stability, Injury. 48 (2017) 2762-2767. doi:10.1016/j.injury.2017.10.029.

[6] M. Bhandari, P.J. Devereaux, T.A. Einhorn, L. Thabane, E.H. Schemitsch, K.J. Koval, F. Frihagen, R.W. Poolman, K. Tetsworth, E. Guerra-Farfán, K. Madden, S. Sprague, G. Guyatt, Hip fracture evaluation with alternatives of total hip arthroplasty versus hemiarthroplasty (HEALTH): Protocol for a multicentre randomised trial, BMJ Open. 5 (2015) 1-12. doi:10.1136/bmjopen-2014-006263.

[7] T. Rezen, A. Kovanda, O. Eiken, I.B. Mekjavic, B. Rogelj, Expression changes in human skeletal muscle miRNAs following 10 days of bed rest in young healthy males, Acta Physiol. 210 (2014) 655-666. doi:10.1111/apha.12228.

[8] E.N. Kubiak, M.J. Beebe, K. North, R. Hitchcock, M.Q. Potter, Early weight bearing after lower extremity fractures in adults., J. Am. Acad. Orthop. Surg. 21 (2013) 727-38. doi:10.5435/JAAOS-21-12-727.

[9] F.K. Ruiz, M.C. Fu, D.D. Bohl, J.W. Hustedt, M.R. Baumgaertner, M.P. Leslie, J.N. Grauer, Patient Compliance With Postoperative Lower Extremity Touch-down Weight-bearing Orders at a Level I Academic Trauma Center, Orthopedics. 37 (2014) e552-e556. doi:10.3928/01477447-20140528-55.

[10] B.J. Braun, N.T. Veith, M. Rollmann, M. Orth, T. Fritz, S.C. Herath, J.H. Holstein, T. Pohlemann, Weightbearing recommendations after operative fracture treatment - fact or fiction? Gait results with and feasibility of a dynamic, continuous pedobarography insole, Int. Orthop. (2017) 1-6. doi:10.1007/s00264-017-3481-7.

[11] A. Vasarhelyi, T. Baumert, C. Fritsch, W. Hopfenmüller, G. Gradl, T. Mittlmeier, Partial weight bearing after surgery for fractures of the lower extremity - is it achievable?, Gait Posture. 23 (2006) 99-105. doi:10.1016/j.gaitpost.2004.12.005.

[12] J.W. Hustedt, D.J. Blizzard, M.R. Baumgaertner, M.P. Leslie, J.N. Grauer, Is it possible to train patients to limit weight bearing on a lower extremity?, Orthopedics. 35 (2012) e31-7. doi:10.3928/01477447-20111122-14.

[13] D. Endicott, R. Roemer, S. Brooks, H. Meisel, Leg load warning system for the orthopaedically handicapped, Med. Biol. Eng. 12 (1974) 318-321.

[14] H.L.P. Hurkmans, J.B.J. Bussmann, E. Benda, J. a N. Verhaar, H.J. Stam, Techniques for measuring weight bearing during standing and walking, Clin. Biomech. 18 (2003) 576-589. doi:10.1016/S0268-0033(03)00116-5.

[15] C. Cooper, G. Campion, L.J. Melton, Hip Fractures in the Elderly : A World-Wide Projection, Osteoporos. Int. 2 
(1992) 285-289. doi:10.1152/jn.00941.2002.

[16] C.M.M. Peeters, E. Visser, C.L.P. Van De Ree, T. Gosens, B.L. Den Oudsten, J. De Vries, Quality of life after hip fracture in the elderly: A systematic literature review, Injury. 47 (2016) 1369-1382. doi:10.1016/j.injury.2016.04.018.

[17] Falls and Fragility Fracture Audit Programme (FFFAP), National Hip Fracture Database (NHFD), Annual report Royal College of Physicians, 2014.

[18] S.E. Roberts, M.J. Goldacre, Time trends and demography of mortality after fractured neck of femur in an English population, 1968-98: database study, October. $327 \quad$ (2003) 1-5. http://www.sciencedirect.com/science/article/pii/S0040402099005396.

[19] X.L. Griffin, N. Parsons, J. Achten, M. Fernandez, M.L. Costa, Recovery of health-related quality of life in a United Kingdom hip fracture population: The warwick hip trauma evaluation - A prospective cohort study, Bone Jt. J. 97-B (2015) 372-382. doi:10.1302/0301-620X.97B3.35738.

[20] Verenso, Triage Instrument Geriatrische Revalidatiezorg, 2012. www.actiz.nl/stream/versenso-triage-instrumentrevalidatiezorg. 


\section{PART}
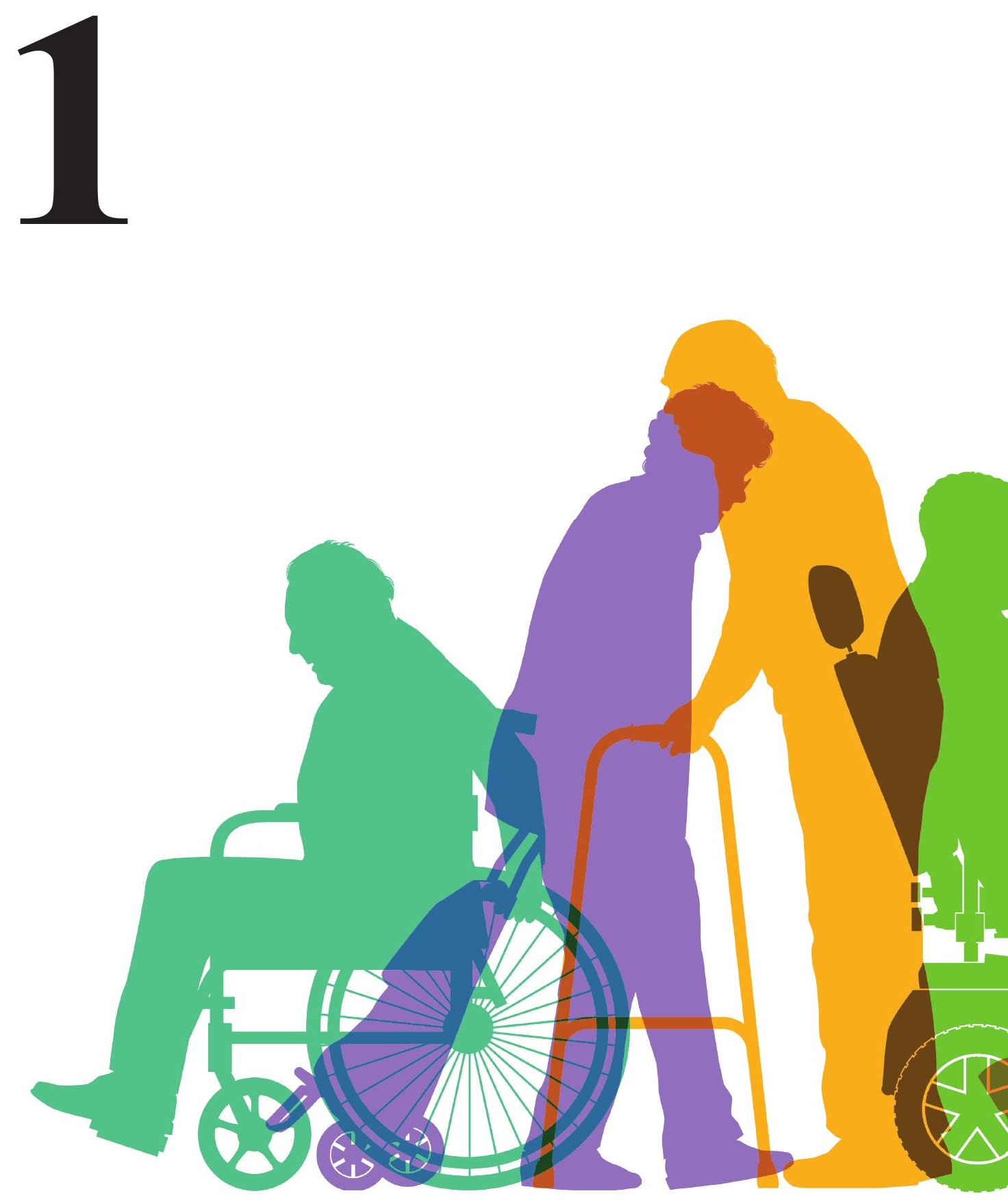
The importance of early weight bearing

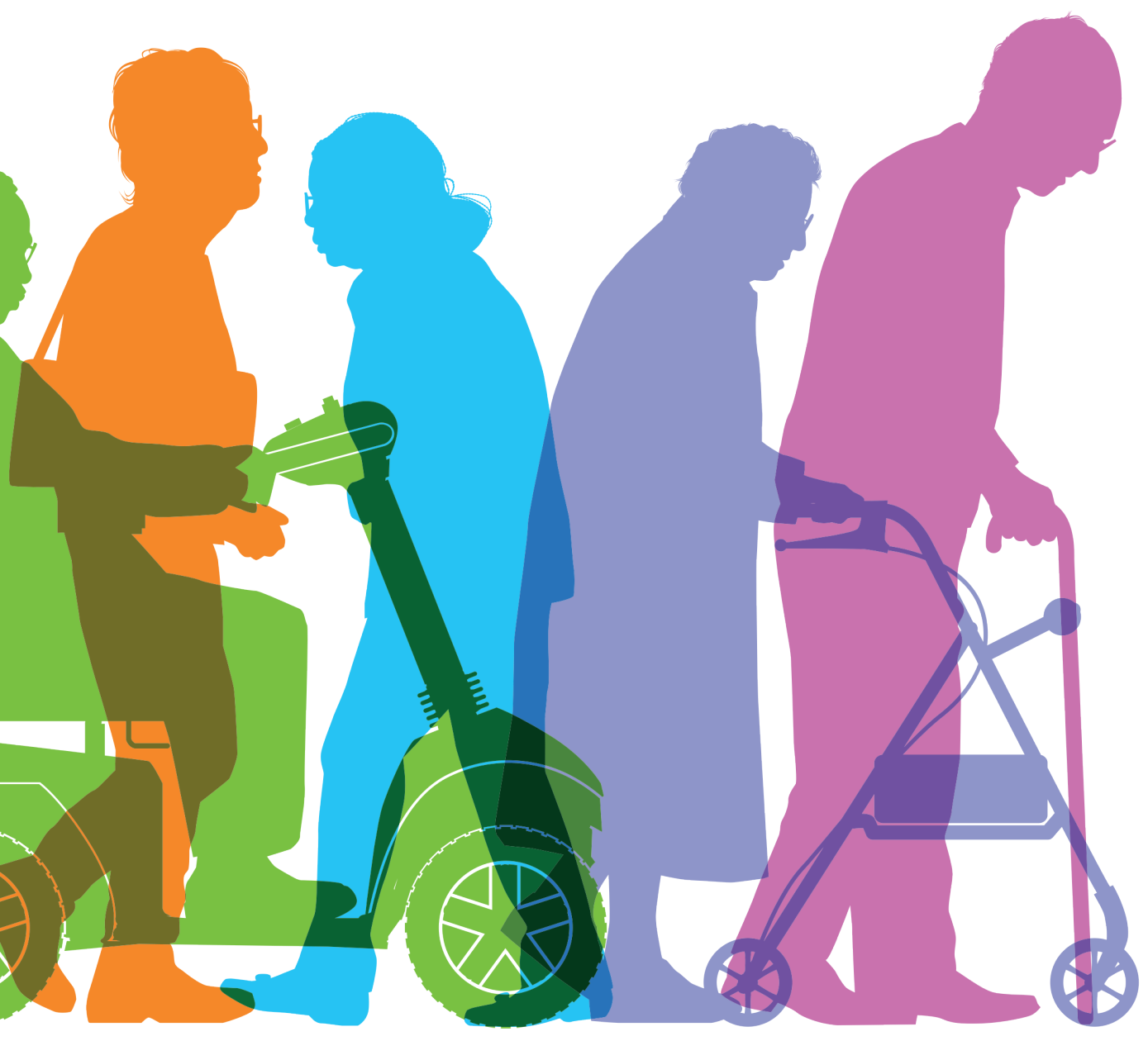


CHAPTER

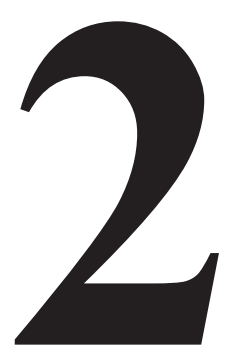


Delay in weight bearing in surgically treated tibial shaft fractures is associated with impaired healing: a cohort analysis of 166 tibial fractures

European Journal of Orthopaedic Surgery \& Traumatology, October 2018, Volume 28, Issue 7, pp 1429-1436

I.B. Houben, M. Raaben, M. Van Basten Batenburg, T.J. Blokhuis 


\begin{abstract}
Background: The relation between timing of weight bearing after a fracture and the healing outcome is yet to be established, thereby limiting the implementation of a possibly beneficial effect for our patients. The current study was undertaken to determine the effect of timing of weight bearing after a surgically treated tibial shaft fracture.

Materials and Methods: Surgically treated diaphyseal tibial fractures were retrospectively studied between 2007 and 2015. The timing of initial weight bearing (IWB) was analysed as a predictor for impaired healing in a multivariate regression.

Results: 166 diaphyseal tibial fractures were included, 86 cases with impaired healing and 80 with normal healing. The mean age was 38.7 years (range 16-89). The mean time until IWB was significantly shorter in the normal fracture healing group (2.6 vs 7.4 weeks, $\mathrm{p}<$ 0.001). Correlation analysis yielded four possible confounders: infection requiring surgical intervention, fracture type, fasciotomy and open fractures. Logistic regression identified IWB as an independent predictor for impaired healing with an odds ratio of 1.13 per week delay (95\% CI 1.03-1.25).

Conclusions: Delay in initial weight bearing is independently associated with impaired fracture healing in surgically treated tibial shaft fractures. Unlike other factors such as fracture type or soft tissue condition, early resumption of weight bearing can be influenced by the treating physician and this factor therefore has a direct clinical relevance. This study indicates that early resumption of weight bearing should be the treatment goal in fracture fixation.
\end{abstract}

Keywords: tibia, fracture, weight bearing, intramedullary nail, fracture healing. 


\section{Introduction}

Impaired healing of tibial fractures remains a challenge in orthopaedic surgery, affecting quality of life and posing economic problems [1,2]. A sophisticated approach in management and stimulation of the bone healing process is necessary to overcome or avoid these problems. The so-called Diamond Concept describes five factors contributing to bone healing: osteogenic cells, osteoinductive stimulants, osteoconductive scaffolds, vascularity and mechanical environment [3]. The first four of these are mainly patient-related and reflect the biological activity of the patient. In contrast, the mechanical environment is under the direct influence of the treating physician, since the mechanical environment is the direct result of treatment options (i.e. a cast or a plate) and decision making (i.e. weight bearing or not). The relevance from a proper mechanical environment finds it origin in several key cellular processes that contribute to bone healing. After activation by external mechanical loading, mechanosensory osteocytes modulate the recruitment and activity of osteoblasts and osteoclasts, leading to remodelling of bone and bone formation through a process referred to as mechanotransduction. The mechanisms of mechanotransduction have been widely explored in in vitro studies [4]. It is known from animal studies that immediate high strain rates, comparable to walking strains, induce a greater amount of callus formation during the early phase of fracture healing [5-7]. Others have shown a dosedependent stimulation of enchondral ossification by interfragmentary strains [8]. Despite these studies, translation of the concept of mechanotransduction to clinical practice has not yet been achieved. A direct relation between mechanical loading and healing outcome has not yet been established.

Current concepts in fracture fixation suggest that micromovement in the fracture should take place and that early weight bearing should be initiated as soon as possible after fracture management [9]. A common argument against early weight bearing is possible loss of reduction. However, a recent biomechanical study has shown that angular stable locking plates may allow full or at least partial immediate weight bearing [10]. A recent randomized controlled trial shows the safety of early weight bearing after intramedullary fixation [11]. Moreover, withholding weight bearing causes loss of bone density. Not loading the bone for eight weeks after a fracture reduces bone density up to one year after the fracture [12]. Additionally, prolonged inactivity quickly affects skeletal muscle tissue [13], leading to decreased mechanosensory function, atrophy, and even increased energy expenditure on 
ambulation [14]. The aim of this study was to explore the relation between timing of weight bearing and fracture healing after tibial shaft fracture surgery. We hypothesized a positive correlation between early initial weight bearing and fracture healing outcome, suggesting that delay of initial weight bearing will increase the rate of impaired healing. In order to assess the safety of weight bearing the fixation failure rate was also analysed.

\section{Materials and Methods}

All consecutive tibial shaft fractures surgically treated in a level-I trauma centre between January 2007 and March 2015 were analysed. Data were retrieved from electronic medical records. Only surgically treated diaphyseal fractures of the tibia were included according to the AO/OTA rule of squares described by Müller et al [15]. All patients with impaired fracture healing (IFH) were identified. IFH was defined as fractures without consolidation (see below) with a minimum duration of at least 6 months after the fracture. In this manner, patients with a delayed union as well as patients with a non-union as defined by the Food and Drug Administration were included [16]. Early radiographic follow-up consisted of an intraoperative or early postoperative tibial multiplane radiograph. Further follow-up radiographic imaging was performed within 6 months and a second time after 12 months postoperatively. Thereafter, follow-up was continued in clinical or radiological impaired healing cases. Patients aged under sixteen years at the time of injury and fractures followed by an amputation within nine months after injury were excluded from this study. If no clinical follow-up was performed at 9 months, patients with IFH were excluded.

Initial weight bearing (IWB) was defined as the first documented moment the patient was able to load the affected leg with any weight exceeding touch-down weight bearing (TDWB), which in earlier studies is defined as loading between 15 and 35 lbs. (6,82- 15,91 $\mathrm{kg})[17,18]$. Healing time was defined as the time between injury and pain-free full weight bearing with radiological signs of progressive consolidation. Radiological union was assessed using radiographs in two orthogonal planes, commonly anteroposterior views and lateral views. When three out of four cortices demonstrated cortical bridging or complete disappearance of fracture lines, radiological union was scored [19]. Additionally, for the non-unions and for delayed unions the healing type was assessed independently by two of the investigators (MR and TB). The radiographs were classified as atrophic or hypertrophic bone healing. Clinical healing was defined as the ability to bear full weight without pain $[20]$. 
Data on body mass index (BMI), injury severity score (ISS), smoking, non-steroidal antiinflammatory drugs (NSAID) use, steroid use, neurological trauma, presence of other orthopaedic injuries, infections requiring surgery, fixation treatment and reaming or nonreaming in IM-nailing surgery were retrieved from medical records. Since previous studies have identified fracture type, fasciotomy and open fractures as clear risk factors for impaired healing [21-24], these risk factors were also included in the analysis. Smoking was scored positive if patients smoked during fracture healing or within six months before trauma, all regardless of quantity. Two drug types were included in order to assess drug use influencing fracture healing; NSAIDs and steroids [25]. The majority of patients received NSAIDs postoperatively for three to seven days. NSAIDs taken over a longer period than one week postoperatively were assessed as drug use influencing fracture healing [26]. Both NSAIDs and steroids were assessed regardless of frequency and quantity. Fracture classification was assessed according to the AO/OTA classification. In order to maintain a reliable assessment only the main categories type $\mathrm{A}, \mathrm{B}$ or $\mathrm{C}$, representing respectively simple, wedged and complex fractures, were used [27]. Open fractures were assessed according to the Gustilo classification [28]. Traumatic brain injury (TBI) was categorized according to the Glasgow Coma Scale (GCS), where a GCS of 13-15 was classified as mild TBI, a GCS of 9-12 as moderate and a GCS of 3-8 as severe [29]. Mild TBI was considered as TBI not affecting rehabilitation, moderate or severe TBI was registered as neurological trauma affecting rehabilitation and was therefore included in the analysis. Furthermore, tibial fracture patients with vertebral injury, pelvic injury, ipsilateral lower extremity injury or contralateral lower extremity injury were documented. This was done in order to assess whether other orthopaedic injuries possibly preventing rehabilitation, increased the chance of developing IFH.

Infections requiring surgery (infection procedures) that occurred within nine months after trauma were included in the primary analysis as a possible risk factor for impaired healing outcome [30]. The following procedures were considered infection surgeries: placement of gentamycin beads, antibiotic coated nail placement, sequestrectomy, total removal of implant material, or debridement. Finally, fixation failure was assessed and comprised both implant failure (assessed in case of radiologic reported bending or breakage of the main implant, including the screws) and loss of reduction with loosening of the implant. 


\section{Statistical analysis}

All data are shown as mean \pm standard deviation (SD), unless otherwise specified. Data were analysed in four steps.

First, data distribution was assessed. Data on BMI and ISS and IWB were all positively skewed and therefore a logarithmic function of these variables was used for further analysis. Data on age were moderately positively skewed and therefore a square root transformation was calculated and used. Second, the correlation between our predictor (initial weight bearing) and our outcome variable (IFH) was assessed.

Third, the role of possible confounders in the relation with both the predictor and the outcome variable was examined using separate bivariate correlation tests. Three evident literature-based risk factors for developing IFH were used in the regression analyses (see final step) as possible confounders, regardless of their outcome during correlation tests. These were fracture type, fasciotomy, and open fractures [21-24].

As a final step regression analysis was performed to test the predictive value of IWB on the healing outcome in a multivariate analysis. All possible confounders were stepwise added (forward) and dropped (backward) from the regression model to see whether the predictive value of our predictor (IWB) was influenced.

All tests were two-sided with a significance set at $p<0.05$. Data were analysed using SPSS version 23.0 (IBM SPSS Statistics for Windows, Version 23.0, Armonk, NY).

\section{Results}

In 1200 patients tibial fracture in one or both limbs was diagnosed, including all diaphyseal and metaphyseal fractures. 608 of these were tibial shaft fractures. Following our exclusion criteria, a total number of 166 surgically treated diaphyseal tibial fractures were treated in 161 patients. Impaired fracture healing (IFH) occurred in 86 fractures and 80 fractures healed uneventful (normal fracture healing, NFH). Of the impaired fractures, 41 were delayed unions and 45 were non-unions. Age, gender, smoking status and drug use were similar in both healing groups (Table 1). Data on smoking status and drug use were documented in 116 and 151 cases respectively. In total, 93 fractures were open and 73 were closed. Of the open fractures 58 occurred in the impaired healing group. 28 fractures in the impaired healing group were closed. All demographic data are summarized in Table 1. Evident differences in weight bearing were seen between the NFH group and the IFH group 
(Figure 1). Initial weight bearing was significantly later in the IFH group (7.4 weeks \pm SD 10.3) than in the NFH group (2.6 weeks \pm SD 2.8) $\mathrm{R}=0.301, \mathrm{p}<0.001$, Table 2). Among the non-union patients, $18(40 \%)$ had an atrophic type of non-union. This was similar to the delayed union group (17 patients, 41\%). For the non-union group initial weight bearing showed no significant correlation to the type of non-union $(\mathrm{p}=0.892)$. Between both delayed and non-union groups and within the groups, no significant differences were found between smokers and healing type (lowest $\mathrm{p}$-value $=0.384$ ).

\begin{tabular}{|c|c|c|c|c|}
\hline Patient characteristics & $\begin{array}{l}\text { Normal Healing } \\
(\mathrm{n}=\mathbf{8 0})\end{array}$ & $\begin{array}{l}\text { Impaired Healing } \\
(\mathrm{n}=86)\end{array}$ & $\begin{array}{l}\text { Bivariate p- } \\
\text { value } \\
\text { IWB }\end{array}$ & $\begin{array}{l}\text { Bivariate p-value } \\
\text { healing outcome }\end{array}$ \\
\hline Age, mean years $( \pm$ SD $)$ & $36.8(19.0)$ & $40.5(17.9)$ & 0.601 & 0.146 \\
\hline Gender Male; Female & $51 ; 26$ & $66 ; 20$ & 0.931 & 0.095 \\
\hline $\begin{array}{l}\text { BMI, median } \\
\text { (interquartile range) * }\end{array}$ & $23.4(21.2-25.1)$ & $25.8(23.4-27.8)$ & 0.323 & 0.010 \\
\hline Smoking status & & & 0.514 & 0.295 \\
\hline Smoking & 23 & & & \\
\hline Non-smoking & 27 & 42 & & \\
\hline Unknown & 30 & 20 & & \\
\hline Drug use & & & 0.746 & 0.206 \\
\hline NSAIDs & 7 & 14 & & \\
\hline Steroids & 2 & 2 & & \\
\hline Both & - & 2 & & \\
\hline No drug use & 65 & 59 & & \\
\hline Unknown & 6 & 9 & & \\
\hline
\end{tabular}

Table 1. Demographics, smoking status and drug use of cases with normal (within six months) or impaired diaphyseal tibial fracture healing. Correlation analysis with initial weight bearing (IWB) and with healing outcome was done for each variable. Significant correlation is marked in italic font. * 59 cases had missing data.

Bivariate analysis identified a significant correlation of two variables with both initial weight bearing (in weeks) and IFH, being fracture type $(\mathrm{R}=0.380, \mathrm{p}<0.001)$ and infection requiring surgical procedure $(\mathrm{R}=0.298, \mathrm{p}<0.001)$. These variables were therefore identified as a possible confounder. P-values on correlations with other variables as stated 


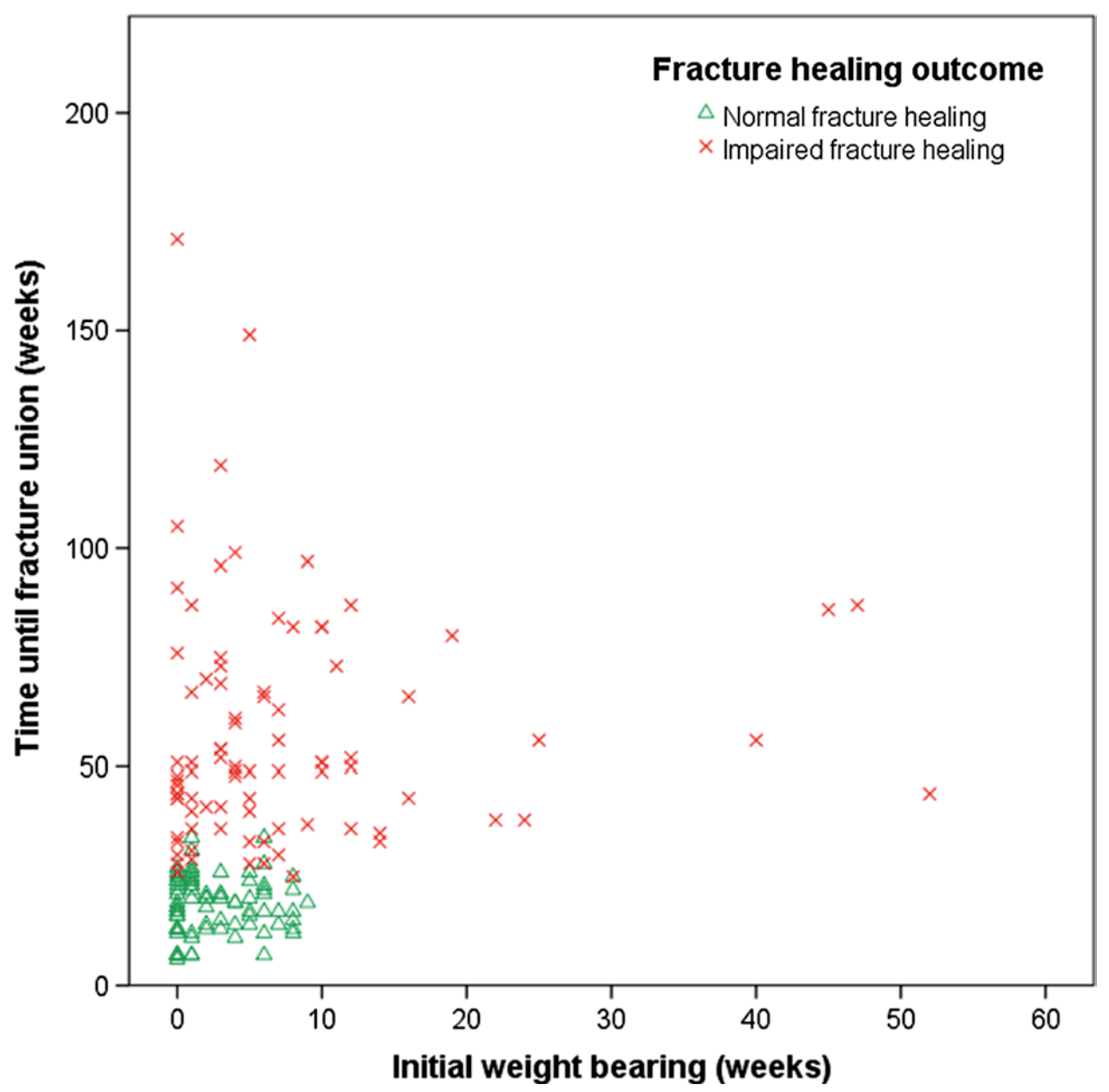

Figure 1. A scatter plot with the timing of initial weight bearing (IWB) in weeks on the $x$-axis and weeks until fracture union after trauma on the $y$-axis. The plot is clustered for fracture healing outcome. The green triangles represent normal fracture healing, and the red crosses represent impaired fracture healing.

in the method section can be found in Table 2. Although the distribution of these factors was similar in our groups, fasciotomy and open fractures were already identified as possible confounders based on literature (together with fracture type) [21-24]. In bivariate analysis these two variables showed relatively high correlations with fracture healing $(\mathrm{R}=0.319$ and 0.239 respectively). Although no significance was found in correlations with both IWB and IFH outcome, they were included in the logistic regression model as possible confounders to isolate the relationship of initial weight bearing and IFH. Following a significant correlation after bivariate analysis $(\mathrm{R}=0,298,=\mathrm{p}<0.001)$, infection requiring surgery was 


\begin{tabular}{|c|c|c|c|c|}
\hline Patient characteristics & $\begin{array}{l}\text { Normal } \\
\text { healing } \\
(\mathrm{n}=\mathbf{8 0})\end{array}$ & $\begin{array}{l}\text { Impaired healing } \\
(\mathrm{n}=\mathbf{8 6})\end{array}$ & $\begin{array}{l}\text { Bivariate p- } \\
\text { value } \\
\text { IWB }\end{array}$ & $\begin{array}{l}\text { Bivariate p- } \\
\text { value } \\
\text { healing outcome }\end{array}$ \\
\hline ISS, mean & 14.8 & 15.4 & 0.440 & 0.844 \\
\hline AO/OTA Type of fracture & & & 0.001 & $<0.001$ \\
\hline Simple & 44 & 23 & & \\
\hline Wedge & 29 & 26 & & \\
\hline Complex & 7 & 37 & & \\
\hline Open fracture & 35 & 58 & 0.168 & 0.002 \\
\hline $\begin{array}{l}\text { Other orthopaedic injuries affecting } \\
\text { rehabilitation }\end{array}$ & & & 0.006 & 0.213 \\
\hline Ipsilateral lower extremity fracture & 7 & 2 & & \\
\hline Both lower extremities fractured & 7 & 15 & & \\
\hline Pelvic injury & 1 & 2 & & \\
\hline Vertebral injury & 2 & 5 & & \\
\hline Polytrauma ( $>2$ of above-named injuries) & 5 & 5 & & \\
\hline $\begin{array}{l}\text { Neurological trauma affecting } \\
\text { rehabilitation }\end{array}$ & 9 & 10 & 0.408 & 0.939 \\
\hline & & & $<0.001$ & 0.080 \\
\hline Intramedullary nailing & 76 & 75 & & \\
\hline Open Reduction Internal Fixation & 4 & 11 & & \\
\hline IM nails reamed $(\%)$ * & 38.9 & 56.6 & 0.329 & 0.067 \\
\hline Fasciotomy & 5 & 27 & 0.070 & $<0.001$ \\
\hline Infection procedures & 1 & 17 & 0.009 & $<0.001$ \\
\hline $\begin{array}{l}\text { Duration of fracture healing, mean in } \\
\text { weeks }( \pm \mathrm{SD})\end{array}$ & $18.4(6.5)$ & $57.1(26.5)$ & $<0.001$ & - \\
\hline Timing of IWB, mean in weeks $( \pm$ SD) & $2.6(2.8)$ & $7.4(10.3)$ & - & $<0.001$ \\
\hline
\end{tabular}

Table 2. Trauma-, fracture- and management characteristics of cases with normal (within six months) or impaired diaphyseal tibial fracture healing. Correlation analysis with initial weight bearing (IWB) and with healing outcome was done for each variable. Significant correlation is marked in italic font. * 59 cases had missing data. 
also added to the model as a possible confounder. A logistic regression was performed to ascertain the effect of initial weight bearing on the likelihood of IFH occurring. The logistic regression model showed a significant relation between initial weight bearing and healing outcome $\left(\chi^{2}(5)=57.338, \mathrm{p}<0.001\right)$. The odds ratio $(\mathrm{OR})$ of developing IFH was 1.13 per week delay in resumption of weight bearing (95\% Confidence Interval: 1.03-1.25). The model correctly classified $75.3 \%$ of cases and explained $39.0 \%$ (Nagelkerke R2) of the variance in fracture healing. Forward and backward stepwise regression showed that this relation was not influenced by any of the identified variables. Hence, while adjusted for four possible confounders, initial weight bearing significantly predicted IFH $(p=0.012)$. Regression coefficients and standard errors are shown in Table 3.

Fixation failure occurred in sixteen cases. Thirteen of these sixteen fixation failures concerned screw breakage in IM-nails. One of all sixteen failures concerned breakage of the nail and one concerned breakage of the plate. Screw bending without breakage occurred in one patient.

In one patient fixation failure occurred early after fracture fixation in a non-compliant patient. All other patients that showed fixation failure had started weight bearing at least one month prior to fixation failure. Mean time until fixation failure was 6 months $( \pm \mathrm{SD}$ 4.63 months) after injury (range 1 - 15.5 months including two outliers after 12 and 15.5 months).

Of all patients, 75 cases required additional surgery. Of these 75 cases, the number of additional procedures per case was 2.32 with a range of 1-11 procedures. Eighteen out of 75 patients required at least one additional procedure based on surgical infection management within nine months after primary fixation (Table 2). These procedures were removal of osteosynthetics (4 patients), sequestrectomy (3 patients), deep cleaning and drainage of the fracture area ( 9 patients) and/or gentamicin beads or gentamicin coated nail placement ( 7 patients). There were patients with infection having a combination of these procedures. Twenty-seven out of 75 cases received removal of screws for dynamization purposes to improve healing. In the remaining 20 cases additional surgery consisted of superficial wound cleaning or removal of screws, because of skin damage or patient complaints. 


\begin{tabular}{|c|c|c|c|c|}
\hline & \multirow[t]{2}{*}{ p-value } & \multirow[t]{2}{*}{ Odds Ratio } & \multicolumn{2}{|c|}{ 95\% CI for OR } \\
\hline & & & Lower & Upper \\
\hline Initial weight bearing & 0.012 & 1.13 & 1.03 & 1.25 \\
\hline Infection requiring procedure & 0.143 & 5.23 & 0.571 & 48.01 \\
\hline Fracture type & 0.002 & 2.19 & 1.34 & 3.58 \\
\hline Fasciotomy & 0.040 & 3.39 & 1.06 & 10.84 \\
\hline Open fracture & 0.109 & 1.84 & 0.87 & 3.87 \\
\hline
\end{tabular}

Table 3. Summary of multivariate logistic regression analysis with impaired fracture healing as our dependent outcome variable.

\section{Discussion}

Weight bearing after a fracture is important, as it helps to maintain bone and muscle mass, and helps to return to daily life participation $[13,14]$. Moreover, through a process referred to as mechanotransduction weight bearing supports bone healing. This positive effect, however, has been determined in laboratory based studies mostly [5,6,8] and many surgeons are still hesitant to permit immediate weight bearing. This study showed a clear relation between healing outcome and initiation of weight bearing in 166 consecutive surgically treated tibial shaft fractures. In this cohort study a significant correlation between timing of initial weight bearing and outcome in diaphyseal tibial fracture healing was identified. Patients who showed normal healing of their tibial fractures started initial weight bearing significantly sooner than patients who developed impaired healing. Moreover, in a multivariate analysis late resumption of weight bearing was identified as an independent risk factor for development of impaired healing. Although the odds ratio for development of impaired healing is relatively small, it accounts for an odds ratio per week delay of weight bearing restriction, indicating a cumulative effect per week extra delay. Other risk factors are present and play an evident role, but in contrast to many other factors, weight bearing is under the direct influence of the treating physician.

Mechanical stability of the current used osteosynthetic material allows for full weight bearing and the average load supported by the injured limb in tibial fracture patients exceeds $50 \%$ of the uninjured limb at one week without any fixation failure $[9,10]$. More recently the safety of early weight bearing in tibial shaft fractures was demonstrated in a prospective study on early weight bearing. Although fixation failure is often mentioned as 
an argument in postponing initial weight bearing, only sixteen patients (9.6\%) of our total population developed a fixation failure. The majority consisted of inconsequently and costneutral bending or breakage of screws in IM-nails, also referred to as autodynamization. In these cases reoperation was not necessary. Considering the small number of clinically relevant fixation failures $(1.8 \%)$ and the timing of failure ( $\sigma 6$ months), fixation failure in this population is considered a result of impaired healing, instead of a cause. Safety is therefore not considered a valid argument against resumption of weight bearing in this population.

Given the retrospective design of our study, several limitations have to be kept in mind when interpreting this data. First, timing of initial weight bearing was retrieved from the electronic medical records. For each patient initial weight bearing was defined as any loading exceeding touch-down weight bearing (TDWB) and the timing was identified based on notes of the doctor or the physical therapist. The physical therapists are all experienced and exclusively appointed to the trauma ward, but training of patients and documentation of progress is not necessarily performed every day. Thereby underreporting of loading may play a role in the data. Since reporting is generally done more extensively in patients requiring more care, this effect is expected to be larger in the normal healing group. Second, as the definition of impaired fracture healing is still debated upon, delayed healing and non-unions were combined. This method may cause an overestimation of actual union healing complication, because many patients with delayed healing may have a seemingly natural healing course. Although this increases the heterogeneity of the impaired healing group, combining delayed and non-unions has increased the number of cases and resulted in increased analytical strength of the study. We realize that a non-union rate of $27 \%$ is high, compared to an average reported rate ranging from 5 to $17 \%[2,11,22,30]$. In contrast to the majority of trials, our series consisted of surgically treated tibial fractures encountered in a level-I trauma center with relatively many open injuries and can therefore not be seen as representative for any general orthopaedic practice population. Moreover, this population has been injured mostly in high-energy trauma, which may be an independent risk factor for bone healing [31]. In our group, no difference in atrophic or hypertrophic healing pattern was seen in the impaired healing group compared to the nonunion group, but the number of cases is relatively small to warrant a conclusion in this perspective. Third, the number of cases included in the final analysis is relatively small compared to the total number of patients treated during the study period. This is due to 
several factors, including treatment in a level-I facility, where patients are transferred to other institutions after initial stabilization and thereby are lost to follow-up. Finally, our data do not represent healing of all tibial fractures. Only surgically treated tibial shaft fractures were included. This has limited the sample size of our study and limits the external validity of our data for other fractures, such as non-operatively treated tibial shaft fractures. However, the homogeneity in our study group was increased by this inclusion, leading to improved rigidity of our conclusions.

Another consideration in interpretation of this study is the possibility of a reverse relationship between weight bearing and healing outcome; delayed healing may result in more pain, and therefore delay weight bearing, instead of delayed weight bearing leading to impaired healing. Two arguments are important in this perspective. First, the delay in weight bearing for both normal healing and impaired healing (2.6 and 7.4 weeks after surgery, respectively) is for both groups much shorter than the mean time to union, even for normal healing. In other words, delay in weight bearing is observed in the early phase of fracture healing, which is consistent with the concept of mechanotransduction. Second, healing outcome was defined as the dependent variable throughout the statistical evaluation. In both forward and backward logistic regression the time of initial weight bearing was identified as an independent factor with a significant predictive value on healing outcome $(p=0.012)$. This rigorous statistical analysis indicates a clear effect of early weight bearing on positive healing outcome.

\section{Conclusion}

In conclusion, the current study is the first to describe the relation between delay in weight bearing and development of impaired healing of surgically treated tibial shaft fractures. A significant relation was observed $(\mathrm{p}=0.012)$, indicating a risk of impaired healing that increases with each week in delay of weight bearing. The aim in treating tibial shaft fractures should therefore be to allow early weight bearing, exceeding touch-down weight bearing, as soon as possible. 


\section{References}

[1] Brinker MR, Hanus BD, Sen M, O'Connor DP. The devastating effects of tibial nonunion on health-related quality of life. J Bone Jt Surg. 2013;95(24):2170-2176. doi:10.2106/JBJS.L.00803.

[2] Dahabreh Z, Calori GM, Kanakaris NK, Nikolaou VS, Giannoudis P V. A cost analysis of treatment of tibial fracture nonunion by bone grafting or bone morphogenetic protein-7. Int Orthop. 2009;33(5):1407-1414. doi:10.1007/s00264-008-0709-6.

[3] Giannoudis P V., Einhorn TA, Schmidmaier G, Marsh D. The diamond concept - open questions. Injury. 2008;39(SUPPL.2). doi:10.1016/S0020-1383(08)70010-X.

[4] Klein-Nulend J, Bakker AD, Bacabac RG, Vatsa A, Weinbaum S. Mechanosensation and transduction in osteocytes. Bone. 2013;54(2):182-190. doi:10.1016/j.bone.2012.10.013.

[5] Bailón-Plaza A, Van Der Meulen MCH. Beneficial effects of moderate, early loading and adverse effects of delayed or excessive loading on bone healing. J Biomech. 2003;36(8):1069-1077. doi:10.1016/S0021-9290(03)00117-9.

[6] Goodship AE, Cunningham JL, Kenwright J. Strain rate and timing of stimulation in mechanical modulation of fracture healing. Clin Orthop Relat Res. 1998;(355S):S105-S115. doi:10.1097/00003086-199810001-00012.

[7] Yamaji T, Ando K, Wolf S, Augat P, Claes L. The effect of micromovement on callus formation. J Orthop Sci. 2001;6(6):571-575. doi:10.1007/s007760100014.

[8] Claes LE, Heigele CA, Neidlinger-Wilke C, et al. Effects of Mechanical Factors on the Fracture Healing Process. Clin Orthop Relat Res. 1998;355S(355 Suppl):S132-S147. doi:10.1097/00003086-199810001-00015.

[9] Koval KJ, Sala DA, Kummer FJ, Zuckerman JD. Postoperative weight-bearing after a fracture of the femoral neck or an intertrochanteric fracture. $J$ Bone Joint Surg Am. 1998;80(3):352-356. http://www.ncbi.nlm.nih.gov/pubmed/9531202. Accessed October 5, 2017.

[10] Carrera I, Gelber PE, Chary G, GonzÃ $j$ lez-Ballester MA, Monllau JC, Noailly J. Fixation of a split fracture of the lateral tibial plateau with a locking screw plate instead of cannulated screws would allow early weight bearing: a computational exploration. Int Orthop. 2016;40(10):2163-2169. doi:10.1007/s00264-015-3106-y.

[11] Gross SC, Galos DK, Taormina DP, Crespo A, Egol KA, Tejwani NC. Can Tibial Shaft Fractures Bear Weight After Intramedullary Nailing? A Randomized Controlled Trial Level of Evidence: Therapeutic Level I. See Instructions for Authors for a complete description of levels of evidence. J Orthop Trauma. 2016;30:370-376. doi:10.1097/BOT.0000000000000598.

[12] Kazakia GJ, Tjong W, Nirody JA, et al. The influence of disuse on bone microstructure and mechanics assessed by HR-pQCT. Bone. 2014;63:132-140. doi:10.1016/j.bone.2014.02.014.

[13] Rezen T, Kovanda A, Eiken O, Mekjavic IB, Rogelj B. Expression changes in human skeletal muscle miRNAs following 10 days of bed rest in young healthy males. Acta Physiol. 2014;210(3):655-666. doi:10.1111/apha.12228.

[14] Xia L, Cheung K-K, Yeung SS, Yeung EW. The involvement of transient receptor potential canonical type 1 in skeletal muscle regrowth after unloading-induced atrophy. J Physiol. 2016;594(11):3111-3126. doi:10.1113/JP271705.

[15] Müller ME, Koch P, Nazarian S, Schatzker J. The Comprehensive Classification of Fractures of Long Bones. 1st editio. Berlin Heidelberg: Springer-Verlag; 1990. doi:10.1007/978-3-642-61261-9. 
[16] Harwood PJ, Newman JB, Michael ALR. (ii) An update on fracture healing and non-union. Orthop Trauma. 2010;24(1):9-23. doi:10.1016/j.mporth.2009.12.004.

[17] Hustedt JW, Blizzard DJ, Baumgaertner MR, Leslie MP, Grauer JN. Lower-extremity Weight-bearing Compliance Is Maintained Over Time After Biofeedback Training. Orthopedics. 2012;35(11):e1644-e1648. doi:10.3928/01477447-20121023-24.

[18] Ruiz FK, Fu MC, Bohl DD, et al. Patient compliance with postoperative lower extremity touch-down weightbearing orders at a level I academic trauma center. Orthopedics. 2014;37(6):e552-6. doi:10.3928/01477447-20140528-55.

[19] Whelan DB, Bhandari M, Stephen D, et al. Development of the Radiographic Union Score for Tibial Fractures for the Assessment of Tibial Fracture Healing After Intramedullary Fixation. J Trauma Inj Infect Crit Care. 2010;68(3):629-632. doi:10.1097/TA.0b013e3181a7c16d.

[20] Joslin CC, Eastaugh-Waring SJ, Hardy JRW, Cunningham JL. Weight bearing after tibial fracture as a guide to healing. Clin Biomech. 2008;23(3):329-333. doi:10.1016/j.clinbiomech.2007.09.013.

[21] Blair JA, Stoops TK, Doarn MC, et al. Infection and Nonunion After Fasciotomy for Compartment Syndrome Associated With Tibia Fractures. J Orthop Trauma. 2016;30(7):392-396. doi:10.1097/BOT.0000000000000570.

[22] Metsemakers WJ, Roels N, Belmans A, Reynders P, Nijs S. Risk factors for nonunion after intramedullary nailing of femoral shaft fractures: Remaining controversies. Injury. 2015;46(8):1601-1607. doi:10.1016/j.injury.2015.05.007.

[23] Teraa M, Blokhuis TJ, Tang L, Leenen LPH. Segmental tibial fractures: An infrequent but demanding injury. Clin Orthop Relat Res. 2013;471(9):2790-2796. doi:10.1007/s11999-012-2739-z.

[24] Santolini E, West R, Giannoudis P V. Risk factors for long bone fracture non-union: A stratification approach based on the level of the existing scientific evidence. Injury. 2015;46:S8-S19. doi:10.1016/S0020-1383(15)30049-8.

[25] Pountos I, Georgouli T, Blokhuis TJ, Pape HC, Giannoudis P V. Pharmacological agents and impairment of fracture healing: What is the evidence? Injury. 2008;39(4):384-394. doi:10.1016/j.injury.2007.10.035.

[26] Kurmis AP, Kurmis TP, O’Brien JX, Dalén T. The Effect of Nonsteroidal Anti-Inflammatory Drug Administration on Acute Phase Fracture-Healing: A Review. J Bone Jt Surg. 2012;94(9):815. doi:10.2106/JBJS.J.01743.

[27] Meling T, Harboe K, Enoksen CH, Aarflot M, Arthursson AJ, Søreide K. How reliable and accurate is the AO/OTA comprehensive classification for adult long-bone fractures? J Trauma Acute Care Surg. 2012;73(1):224-231. doi:10.1097/TA.0b013e31824cf0ab.

[28] Gustilo RB, Anderson JT. Prevention of Infection in the Treatment of One Thousand and Twenty-five Open Fractures of Long Bones. J Bone Jt Surg. 2002;58(4):2002. doi:10.1007/978-1-4471-5451-8_138.

[29] Saatman KE, Duhaime A-C, Bullock R, Maas AIR, Valadka A, Manley GT. Classification of Traumatic Brain Injury for Targeted Therapies. J Neurotrauma. 2008;25(7):719-738. doi:10.1089/neu.2008.0586.

[30] Westgeest J, Weber D, Dulai SK, Bergman JW, Buckley R, Beaupre LA. Factors Associated With Development of Nonunion or Delayed Healing After an Open Long Bone Fracture. J Orthop Trauma. 2016;30(3):149-155. doi:10.1097/BOT.0000000000000488. 
[31] Karhof S, Bastian OW, Van Olden GDJ, Leenen LPH, Kolkman KA, Blokhuis TJ. Impaired Fracture Healing of the Distal Femur after High Energy Trauma. SM J Arthritis Res. 2017;(October). https://www.researchgate.net/publication/321624564. 
CHAPTER
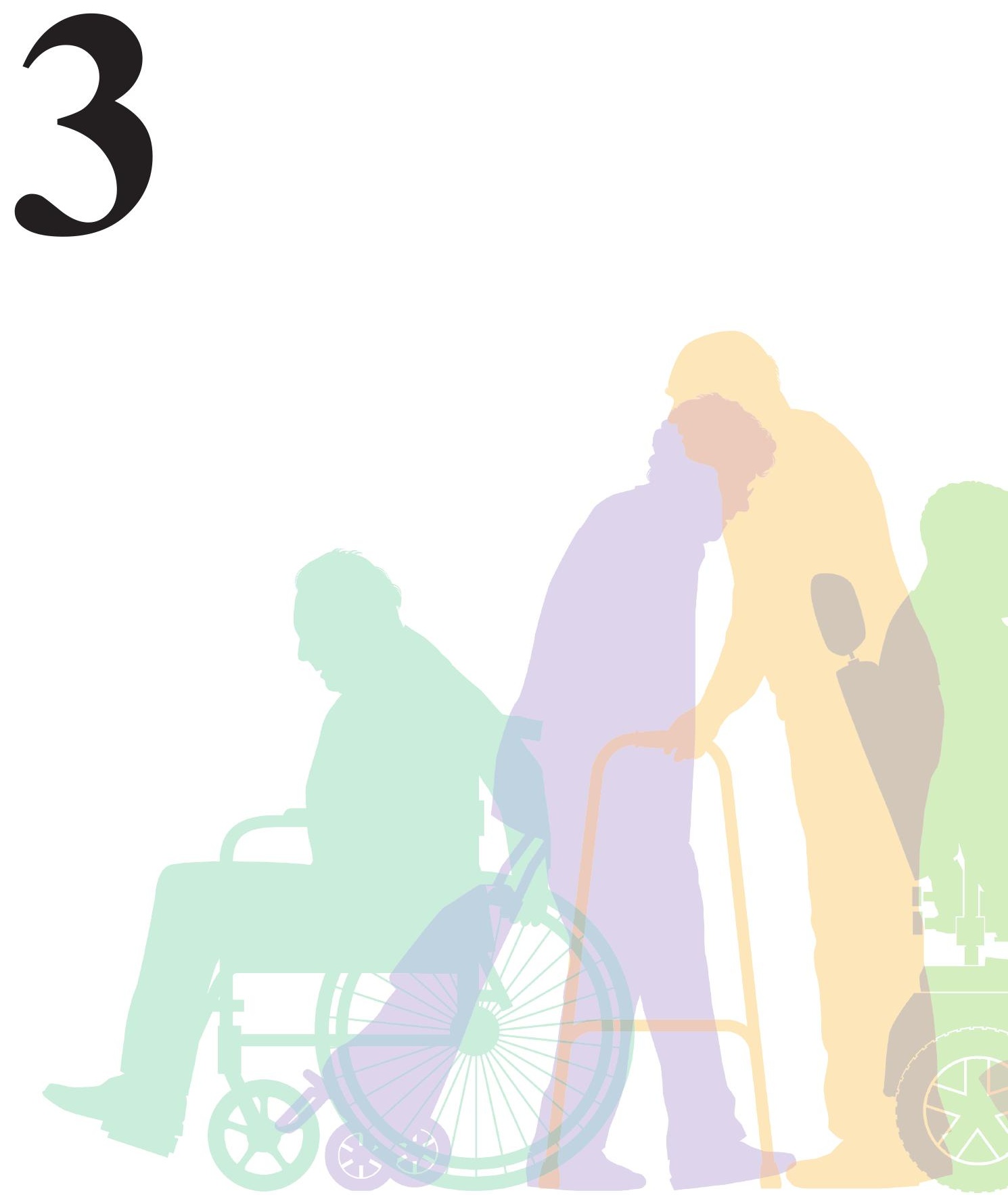
Technical Aspects and Validation of a New Biofeedback System for Measuring Lower Limb Loading in the Dynamic Situation

Sensors, March 2017, Volume 17, Issue 3, pp 658.

M. Raaben, H.R. Holtslag, R. Augustine, R.O. van Merkerk, B.F.J.M. Koopman, T.J. Blokhuis 


\begin{abstract}
Background: A variety of techniques for measuring lower limb loading exists, each with their own limitations. A new ambulatory biofeedback system was developed to overcome these limitations. In this study, we described the technical aspects and validated the accuracy of this system.

Materials and Methods: A bench press was used to validate the system in the static situation. Ten healthy volunteers were measured by the new biofeedback system and a dual-belt instrumented treadmill to validate the system in the dynamic situation.

Results: Bench press results showed that the sensor accurately measured peak loads up to $1000 \mathrm{~N}$ in the static situation. In the healthy volunteers, the load curves measured by the biofeedback system were similar to the treadmill. However, the peak loads and loading rates were lower in the biofeedback system in all participants at all speeds.

Conclusions: Advanced sensor technologies used in the new biofeedback system resulted in highly accurate measurements in the static situation. The position of the sensor and the design of the biofeedback system should be optimized to improve results in the dynamic situation.
\end{abstract}

Keywords: biofeedback, ambulatory monitoring, lower limb loading, weight-bearing. 


\section{Introduction}

Accurate measurements of lower limb loading is relevant in many fields, such as lower limb injuries, diabetes mellitus, stroke, footwear design, sport biomechanics, and injury prevention [1]. Systems for monitoring lower limb loading have therefore become a growing field of interest since the first introduction of such a system in 1974 [1,2]. Current load monitoring systems can be classified in platforms and (ambulatory) biofeedback devices [2].

Force platforms are accurate and easy to use for load measurements in static and dynamic situations [2]. Force platforms placed inside a treadmill are even more beneficial as walking speed can be controlled. Moreover, loading can be controlled by the use of overhead body weight-support systems. The biomechanics of treadmill walking, however, differs from overground walking [3]. Healthy individuals have shown modified muscle activation patterns and thereby modified joint movements and forces during treadmill walking [4]. In addition, greater cadence, smaller stride length and stride time, as well as different ground reaction forces were found in the elderly [5]. Although some ambulatory platforms exist, most platforms are restricted to research laboratories, which prevents clinical implementation $[1,2]$.

The development of biofeedback systems seems promising as lower limb loading can be measured in the dynamic situation. A variety of (semi) portable ambulatory devices are currently available. Although devices such as the F-Scan (Tekscan Inc., Boston, MA, USA), Pedar ${ }^{\circledR}$ Force Monitoring System (Novelgmbh, Munich, Germany), GaitShoe (Massachusetts Institute of Technology (MIT) Media Laboratory, Cambridge, MA, USA) and SmartStep (Andante Medical Devices, Beer Sheva, Israel) have been shown to obtain accurate results [6-10], important limitations still remain. These limitations include the use of cables, sensor migration, creep, temperature drift, humidity drift, hysteresis, limited storage capacity, and a lack of commercial availability [2,11]. Moreover, ambulatory visual feedback about lower limb loading during gait is not possible with any of these systems. At most, an auditory warning signal or postresponse feedback can be given after a training exercise, and this limits the performance and effectiveness for clinical use [12,13]. These drawbacks together with high prices prevent worldwide clinical implementation of these devices. 
To overcome limitations of previous devices, our research group developed a new ambulatory biofeedback system for measuring lower limb loading in the dynamic situation (SensiStep, Evalan BV, Amsterdam, The Netherlands). Important aspects in the development were usability and accuracy for clinical application, as well as the opportunity to provide ambulatory visual feedback in real-time. We aimed to meet the requirements as previously described by Razak et al. [1], including a high level of mobility, limited cabling, correct sensor placement, low power consumption, and low cost. In addition, the system should be able to continuously monitor patients and provide accurate and real-time feedback about lower limb loading to both patient and healthcare professional.

The aim of this first study is to (1) describe the technical aspects of the system and (2) validate the accuracy of the data generated by the system in both static and dynamic situations. Bench press testing was used to validate the system in the static situation. The obtained force measurements in the dynamic situation were compared to a dual-belt instrumented treadmill (R-Mill, ForceLink, Culemborg, The Netherlands).

\section{Materials and Methods}

\section{Technical Aspects of the System}

\section{Set-Up of the System}

The biofeedback system consists of five different components as shown in Figure 1: (1) a force sensor, (2) custom-made sandals, (3) a wrist device, (4) a tablet, and (5) a secured Web Portal. The force sensor (dim: $55 \times 75 \times 14.9 \mathrm{~mm}$, weight: $190 \mathrm{~g}$ ) is placed inside a specific position in the sole of the custom-made sandal. This ensures the sensor is always positioned correctly under the midfoot of the patient. The in-sole sensor sends load measurements wireless and in real-time via Bluetooth 4.0 to a wrist device. This wrist device acts both as data aggregator as well as feedback instrument for the patient. Real-time feedback about lower limb loading is given by the wrist device via green and red light emitting diodes (LEDs). Simultaneously, this device sends the load measurements wireless and in real-time via Bluetooth to a tablet (ASUS Tablet, Model: K01A, ASUSTek COMPUTER INC., Taipei, Taiwan). On this tablet, a specially developed application is installed. This application can be used by the healthcare professional to set the target load, including a range or threshold around this target. During gait, the tablet shows real-time graphic illustrations of all steps, including the predetermined target load. All measurements are saved on a secured Web Portal for postresponse feedback. All electronic components 
contain rechargeable batteries. The components of the system will be discussed in detail in the following sections.

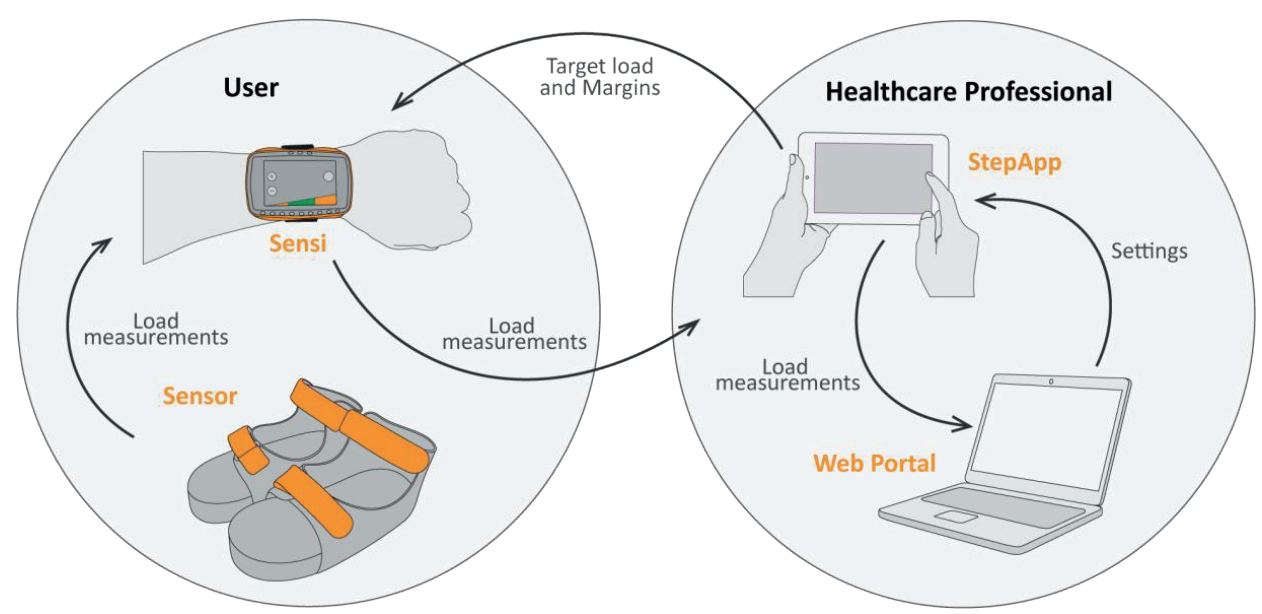

Figure 1. Schematic representation of the biofeedback system with the five components: the insole sensor sends load measurements wireless and in real-time to a wrist device (Sensi), which acts as feedback instrument for the patient. These load measurements are then send wireless and in real-time to a tablet, which acts as feedback instrument for the healthcare professional. The healthcare professional sets the desired target load and margins in the tablet (StepApp). Finally, all sessions are saved on a secured Web Portal.

\section{The Sensor}

The sensor consists of six different parts as shown in Figure 2. The cover plate (1) is connected via small beads to four flat springs (3), which are supported in the center by the bottom plate (2). Magnets (4) and Hall sensors (5) are placed at each corner. Each of these Hall sensors measure the magnetic field created by one of the magnets. A force exerted on the cover plate will be transferred via the beads to the four flat springs. Depending on the applied force, these springs will bend more or less, which results in movement of the corners and thus a change in the magnetic field at each of the Hall sensors. This creates changes in the output signal of the Hall sensors, which makes it possible to calculate the total force exerted on the spring by using the correct signal processing and algorithm. The springs have been designed to behave linearly according to the formula $B=k * \delta$, where $\mathrm{B}$ is the force exerted on the spring, $\mathrm{k}$ is the constant of the spring, and $\delta$ equals the deflection of the spring. Experimental and simulation models have shown that the materials used in the design of the sensor ensure that the bending load is measured (data not shown). By using this sensor, it is possible to measure the axial force exerted on the lower extremities in the dynamic situation. 
The sensor must be calibrated once after manufacturing by applying a constantly increasing force between 0 and $2000 \mathrm{~N}$ (incremental steps of $100 \mathrm{~N}$ ) in the middle of the sensor. Subsequently, data from all four sensors should be recorded independently. For each sensor, a third degree polynomial function can be fitted according to the gathered data and used to calculate the force per sensor. The total force is simply the sum of all four individual sensors. After successful calibration, the sensor can be used without recalibration by the healthcare professional or patient.

\section{$\underline{\text { The Sandals }}$}

The biofeedback system contains custom-made sandals (size 37-40-43-45, range: 35-47). The sensor fits perfectly in the sole of these sandals. This ensures easy and correct sensor placement by the treating physician or patient. The sandals are designed to allow normal gait patterns.

\section{The Wrist Device}

The in-sole sensor sends its load measurements via Bluetooth 4.0 (Bluetooth Low Energy, BLE) to the data aggregator, which is a wrist device worn by the patient. BLE is chosen because it is wireless, has low energy consumption, and has expanding possibilities in the

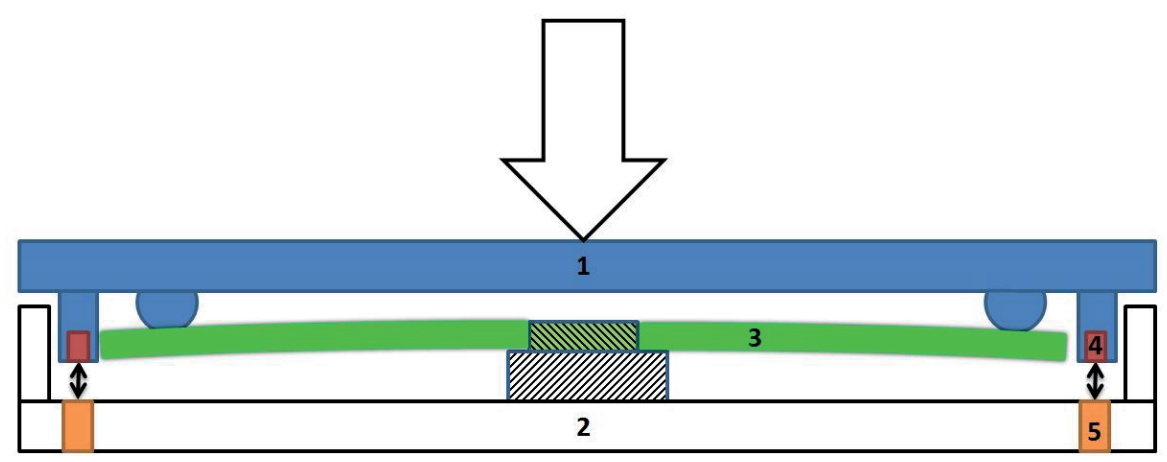

Figure 2. Schematic representation of the sensor. The sensor consists of six different parts: 1) the cover and 2) bottom plate consist of high quality non-ferromagnetic aluminum 7075 (AlZn5,5MgCu), 3) the laser-cut flat spring is made of strong and non-corrosive stainless steel (RVS AISI 301), 4) the N45 magnets are made of NdFeB (Neodymium-IronBoron), 5) the Hall sensors (Honeywell SS49E), and 6) a U3-HV USB data acquisition module (LabJack, Lakewood, CO, USA, not shown in figure). A force exerted on the cover plate (1) will be transferred via the beads to the four flat springs (3). Depending on the applied force, these springs will bend more or less, which results in movement of the magnets (4) and thus a change in the magnetic field at each of the Hall sensors (5). This creates changes in the output signal of the Hall sensors, which makes it possible to calculate the total force exerted on the spring by using the correct signal processing and algorithm. 
future. The wrist device contains two-color LEDs (red/green) that can be programmed to provide real-time feedback about lower limb loading to the patient. The threshold that is set in the tablet (see below) corresponds to these LEDs. Green light means correct loading, and red light means loading below or above the threshold. The wrist device continuously sends data via Bluetooth to the tablet if the two devices are connected.

\section{The Tablet}

The healthcare professional sets the desired target load, including a range around this target, in the application of the tablet. This target load, including range, is shown as a green bar on the tablet screen. Steps are displayed in real-time on the tablet, providing insight in lower limb loading during exercises to both the patient and the healthcare professional. The step detection in the application is a mathematic parameter based on dynamic loading; when a load of $10 \%$ of the target load is measured, a start point is set. If the patient now exceeds at least $20 \%$ of the given target load and lessens the load to below the $10 \%$ line within a time frame of 0.2 to $2.0 \mathrm{~s}$ from the start point, it is registered as a step. For postresponse feedback and/or research purposes, all data are sent via Wi-Fi to a secured Web Portal.

\section{Validation of the Biofeedback System}

\section{Static Loading}

Sensor accuracy was tested in the static situation using a bench press. The experimental design is illustrated in Figure 3. An increasing force ranging from 0 to $1000 \mathrm{~N}$ was applied by the bench press, and the sensor outcome was measured.

\section{Dynamic Loading}

The biofeedback system was also tested in the dynamic situation, and obtained results were compared to a dual-belt instrumented treadmill (R-Mill, ForceLink, Culemborg, The Netherlands). Ten healthy volunteers with unrestricted mobility were equipped with the biofeedback system, consisting of the custom-made sandals, the force sensor, and the wrist device. The tablet was held by the investigator. The participants walked with the biofeedback system on the R-Mill at different speeds: $0.5,1.0,2.0,3.0$, and $4.0 \mathrm{~km} / \mathrm{h}$ for 90s at each speed. All participants gave their informed consent, and the study protocol was approved by the local ethics committee of the University Medical Center Utrecht. 


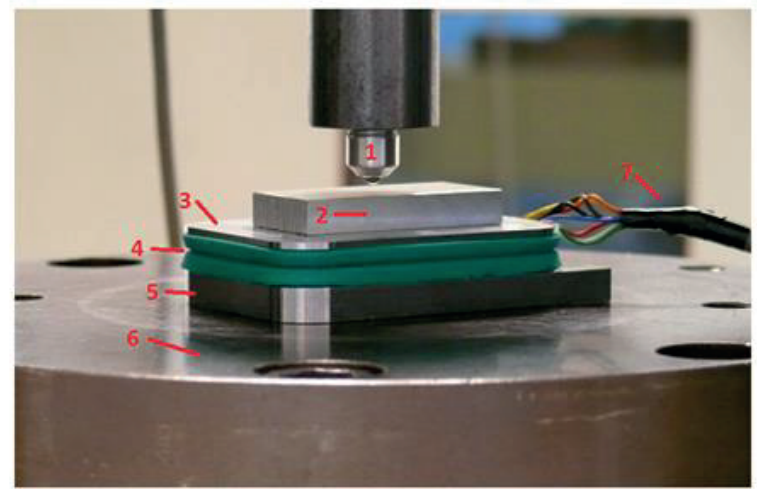

Figure 3. Validation of the sensor in the static situation using a bench press. The sensor (3-5) was placed between a solid underground (6) and a solid plate (2) to increase the area of applied force by the bench press (1). Incremental forces from 0 to $1000 \mathrm{~N}$ were applied to the sensor. For this experimental set-up a cable (7) was connected from the sensor to a laptop to analyze the data in the static situation.

Parameters of interest were peak load and loading rate, as these parameters were considered to be most relevant for this first validation study. Especially the loading rate, which is defined as the steepness of the loading curve, has shown clear correlations with length of stay in the nursing home in a previous study using the FeetB@ck system [14]. Specific Matlab (Matlab R2014a, MathWorks) routines were developed to convert the raw data to parameters of interest. Average peak loads and loading rates were calculated per participant per speed by taking the average of all single steps at that specific speed. The absolute and relative error were then calculated according to the formulas $E(a b s)=F b-F r$ and $E(r e l)=\frac{E(a b s)}{F r}$, respectively, where $\mathrm{E}(\mathrm{abs})$ and $\mathrm{E}(\mathrm{rel})$ are the absolute and relative error, Fr the force measured by the R-mill and $\mathrm{Fb}$ the force measured by the biofeedback system.

\section{Results}

Bench press testing has shown that the sensor accurately measured peak loads up to $1000 \mathrm{~N}$ with a sample frequency of $50 \mathrm{~Hz}$ in static situations at room temperature. A constant applied force on the sensor at increasing temperatures has shown that temperature changes had minimal effects on the stability and accuracy of the sensor between $20{ }^{\circ} \mathrm{C}$ to $37^{\circ} \mathrm{C}$. Cyclic bench press testing has shown that the sensor produced accurate results during cyclic loading for at least 500s. Results of the static validation tests are illustrated in Figure 4. 


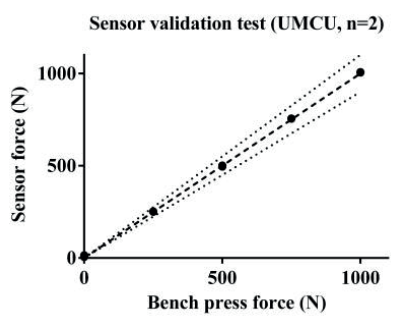

(a)

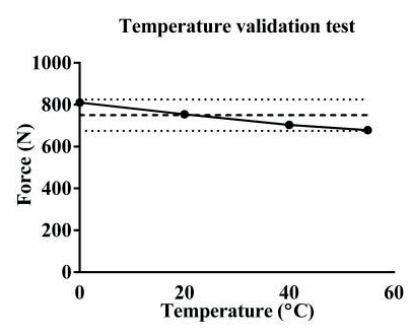

(c)
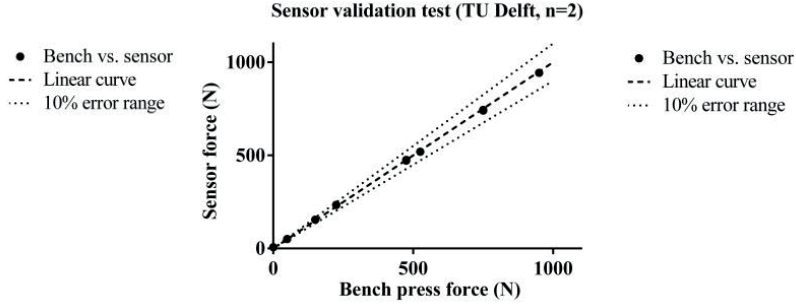

(b)

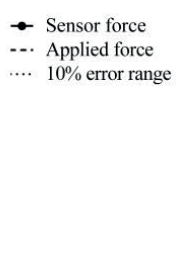

(d)

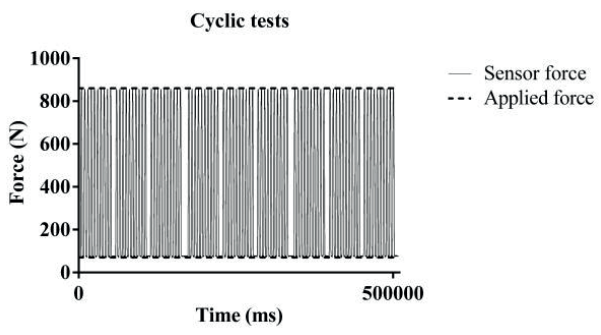

Figure 4. Validation of the sensor in the static situation. $(a, b)$ Tests at the University Medical Center Utrecht $(n=2)$ and Technical University Delft $(n=2)$. The black dots indicate the applied force by the bench press versus the measured force by the sensor; (c) Sensor validation test at a broad range of temperatures $\left(0-55^{\circ} \mathrm{C}\right)$ at constant force $(750 \mathrm{~N})$. The black dots indicate the force measured by the sensor. The intermittent line represents the applied force by the bench press $(750$ $\mathrm{N})$ with the $10 \%$ error range marked by dotted line. In the $20-37{ }^{\circ} \mathrm{C}$ range, the deviation was $1 \%$ at maximum. At extreme temperatures, the error fluctuates between $+8 \%$ and $-9 \%$, but remains within the range of $10 \%$; (d) Cyclic loading at 20 ${ }^{\circ} \mathrm{C}$. Cyclic fluctuating forces between $70 \mathrm{~N}$ and $860 \mathrm{~N}$, highlighted by the intermittent line, were applied to the sensor. The error between applied force and measured force was negligible $(<1 \%)$.

To validate the biofeedback system in the dynamic situation it was tested in 10 healthy volunteers (6 men and 4 women; mean age 24.9 years (SD 2.2 years); weighing $74 \mathrm{~kg}$ (SD $8.0 \mathrm{~kg}$ ) and compared to a dual-belt instrumented treadmill: the R-Mill. The peak loads and loading rates at each speed measured in individual participants are presented in Figure 5. The peak loads measured by the biofeedback system were lower than the peak loads measured by the R-Mill. The same was observed in loading rates, where the difference between the two systems increased with speed. The differences in the two gait parameters are also represented by the load curves in Figure 5. It can be seen that the shape of the load curves were similar, but the biofeedback system curves were lower and less steep than the R-Mill curves. The relative and absolute errors are shown in Tables 1 and 2. 

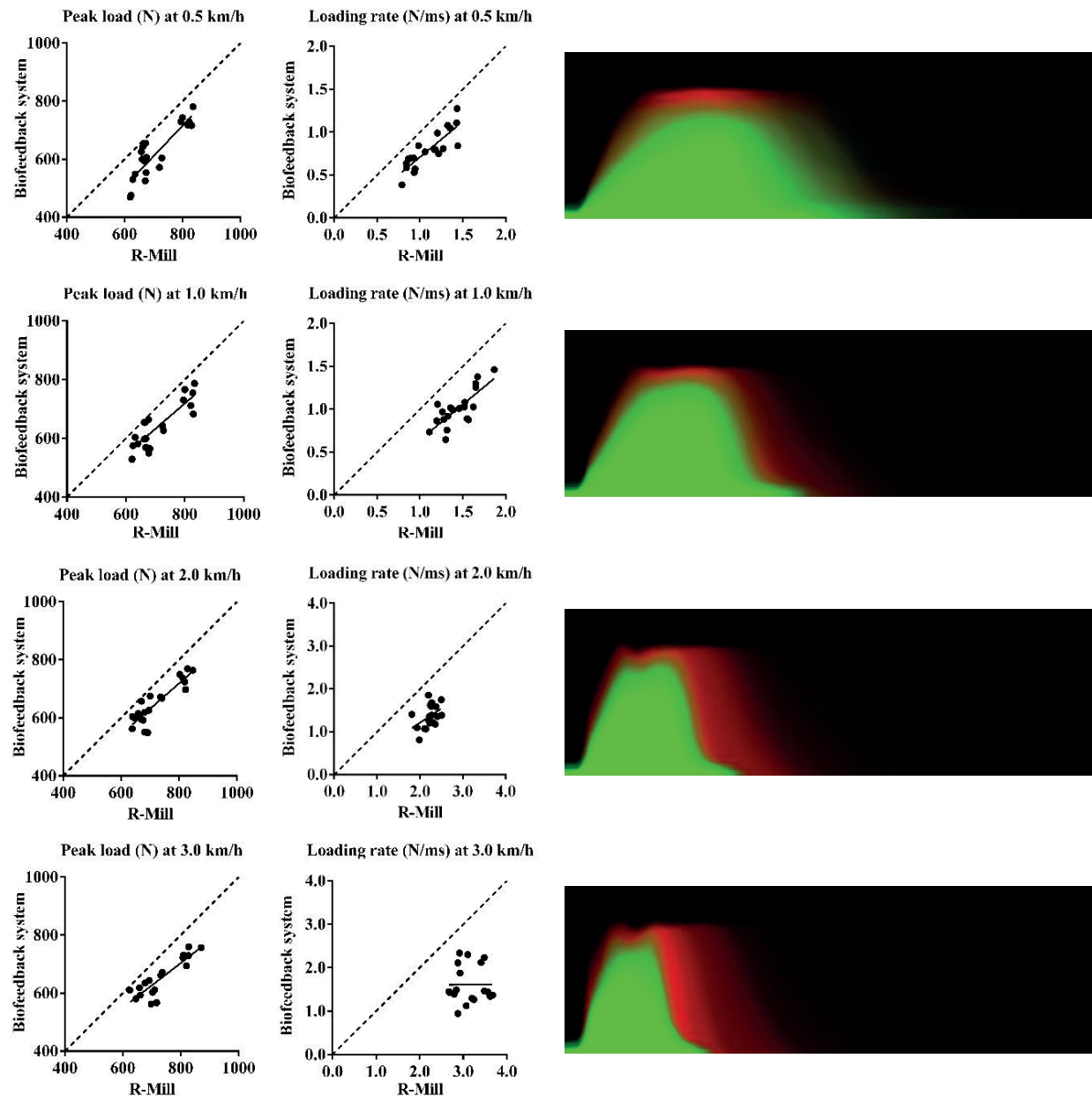

Peak load (N) at $4.0 \mathrm{~km} / \mathrm{h}$

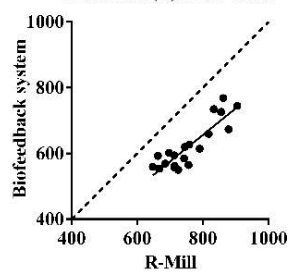

Loading rate (N/ms) at $4.0 \mathrm{~km} / \mathrm{h}$
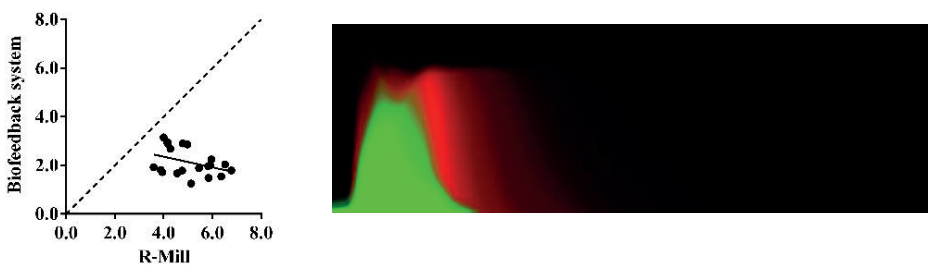

Figure 5. Validation of the biofeedback system in the dynamic situation at different speeds. Left: peak load (N) measured by the R-Mill vs. peak load (N) measured by the biofeedback system. Middle: loading rate (N/ms) measured by the R-Mill vs. loading rate $(\mathrm{N} / \mathrm{ms})$ measured by the biofeedback system. Right: overlay of the load curves $(n=10)$ measured by the RMill (red) and the biofeedback system (green).

Legend: - - - Linear curve, — Observed curve, • R-Mill vs. biofeedback system. 


\begin{tabular}{|l|l|l|}
\hline Speed $\mathbf{( k m / h )}$ & Relative error $(\mathbf{m i n}-\mathbf{m a x})$ & Absolute error in N (min-max) \\
\hline 0.5 & $-0.15(-0.05$ to -0.26$)$ & $-105.9(-34.56$ to -166.5$)$ \\
\hline 1.0 & $-0.12(-0.04$ to -0.21$)$ & $-86.15(-25.14$ to -162.7$)$ \\
\hline 2.0 & $-0.12(-0.04$ to -0.22$)$ & $-97.77(-30.23$ to -166.5$)$ \\
\hline 3.0 & $-0.13(-0.05$ to -0.23$)$ & $-152.2(-86.89$ to -221.3$)$ \\
\hline 4.0 & $-0.20(-0.13$ to -0.27$)$ & \\
\hline
\end{tabular}

Table 1. Average relative and absolute error in peak loads $(\mathrm{n}=10)$.

\begin{tabular}{|l|l|l|}
\hline Speed $(\mathrm{km} / \mathrm{h})$ & Relative error $(\mathrm{min}-\mathrm{max})$ & $-0.03(-0.01$ to -0.06$)$ \\
\hline 0.5 & $-0.29(-0.11$ to -0.51$)$ & $-0.04(-0.02$ to -0.07$)$ \\
\hline 1.0 & $-0.31(-0.12$ to -0.50$)$ & $-0.09(-0.03$ to -0.12$)$ \\
\hline 2.0 & $-0.38(-0.15$ to -0.59$)$ & $-0.16(-0.06$ to -0.24$)$ \\
\hline 3.0 & $-0.48(-0.20$ to -0.67$)$ & $-0.30(-0.09$ to -0.51$)$ \\
\hline 4.0 & $-0.57(-0.21$ to -1.27$)$ & \\
\hline
\end{tabular}

Table 2. Average relative and absolute error in loading rates $(n=10)$.

\section{Discussion}

Measuring lower limb loading in the ambulatory situation has become a growing field of interest in recent decades. Various techniques have been developed, whereby the ambulatory, portable biofeedback systems seem to have the most potential for clinical implementation. Still, existing biofeedback systems suffer from limitations that prevent widespread use in healthcare. Our aim was to develop a new ambulatory biofeedback system that overcomes existing limitations and, in addition, is able to provide real-time feedback about lower limb loading to the patient and the healthcare provider. The development resulted in a biofeedback system that consists of advanced sensor technologies based on the Hall effect. Highly accurate results were obtained in the static situation. The results in the dynamic situation need to be improved in the ongoing development of the system.

Walking is a highly dynamical procedure and therefore difficult to measure. Previous systems encounter problems such as sensor migration, creep, temperature and/or humidity drift and hysteresis [2,11]. These problems can be overcome by using a sensor based on the Hall effect, as correct detection of lower limb loading is ensured, independent of the exact 
loading point on the sensor. In addition, the sensor developed in the current study sends load measurements wireless and in real-time via BLE to a wrist device and tablet. This ensures a high level of mobility, prevents the use of cables, and has low power consumption. These are requirements for a user-friendly biofeedback system, that allows a comfortable, safe and natural gait of the user [1]. Moreover, the system enables the patient and healthcare provider to gain real-time insight in relevant load measurements and thereby the ability to adapt lower limb loading to the optimal level during training.

In this study, the sensor was validated in a static situation and the entire biofeedback system, including the custom-made sandals, wrist device, and tablet, in the dynamic situation. The sensor was highly accurate in the static situation as shown by bench press testing. The sensor has a wide force range from 0 to $1000 \mathrm{~N}$ and shows accurate detection of loading in cyclic testing. Low temperature sensitivity has been observed between $20{ }^{\circ} \mathrm{C}$ and $37^{\circ} \mathrm{C}$, which is considered as the relevant range of daily usage [15].

Data generated by 10 healthy volunteers were compared to a dual-belt instrumented treadmill, as these systems are often used as the gold standard for gait analysis [10]. In this study, we found different results in gait parameters between the biofeedback system and the R-Mill. Important gait parameters as peak load and loading rate registered by the biofeedback system are lower than the data generated by the R-Mill at all speeds. Although it is known that gait patterns on a treadmill differ from overground walking [4], it is more likely that a suboptimal position of the sensor or the use of a single sensor results in lower gait parameters. From previous studies, it is known that plantar forces are different depending on the anatomic region under the foot. For example, peak force increases linearly with speed relative to the heel, lateral toe, and medial toe regions, while no significant differences were found with the midfoot, lateral forefoot, and central forefoot at different speeds [11]. In the experimental set-up of this study, the entire step is measured by the R-Mill, including the heelstrike and toestrike, while the biofeedback system only measured the midstance due to the sensor location under the midfoot region. Probably the plantar force is not entirely measured during heelstrike and toestrike, which lead to the lower gait parameters observed in this study. Possibly, to overcome this issue, multiple sensors covering all anatomic regions under the foot could be applied. Integration of data from multiple sensors, however, is certainly challenging and will result in a less user- 
friendly system. For these reasons, we have chosen to use one sensor in combination with the Hall effect.

With the new biofeedback system previous limitations and problems have been minimized, such as the use of cables, sensor migration, and sensor placement. Results in the static situation as well as the shape of the load curves indicate that the sensor is highly accurate. However, some limitations are still present in this new biofeedback system. The gait parameters measured in the dynamic situation are lower than the gold standard. To overcome this, significant improvements should be made, for example, in the sensor position or in the use of multiple sensors. Improvements can also be made in the design of the custom-made sandals. Previous studies have shown that rigid shoes and insoles have a significant influence on gait parameters [16]. In the ongoing development of the biofeedback system, adjustments in the sandal design are envisaged to overcome, at least in part, these issues. A limitation in the study design is that the system was validated in the static situation by bench press testing and in the dynamic situation by use in healthy volunteers. Clinical application includes patients suffering from lower limb injuries, and data should ideally be validated in this group. Hypothetically, factors as pain and anxiety, lower walking speed and usage of crutches lead to lower results than observed in the healthy volunteers.

\section{Conclusions}

An ambulatory biofeedback system for measuring lower limb loading has been developed that uses advanced sensor technologies. Real-time visual feedback about lower limb loading can be provided to the patient and the healthcare provider. Based on static measurements and the dynamic load curves, it can be concluded that the sensor is highly accurate. Measurements in the dynamic situation, however, indicate that sensor position and sandal design may be improved in further development. 


\section{References}

[1] Abdul Razak, A.H.; Zayegh, A.; Begg, R.K.; Wahab, Y. Foot plantar pressure measurement system: A review. Sensors 2012, 12, 9884-9912.

[2] Hurkmans, H.L.P.; Bussmann, J.B.J.; Benda, E.; Verhaar, J.A.N.; Stam, H.J. Techniques for measuring weight bearing during standing and walking. Clin. Biomech. 2003, 18, 576-589.

[3] Hoogkamer, W.; Bruijn, S.M.; Potocanac, Z.; van Calenbergh, F.; Swinnen, S.P.; Duysens, J. Gait asymmetry during early split-belt walking is related to perception of belt speed difference. J. Neurophysiol. 2015, 114, 1705-1712.

[4] Lee, S.J.; Hidler, J. Biomechanics of overground vs. treadmill walking in healthy individuals. J. Appl. Physiol. 2008, 104, 747-755.

[5] Watt, J.R.; Franz, J.R.; Jackson, K.; Dicharry, J.; Riley, P.O.; Kerrigan, D.C. A three-dimensional kinematic and kinetic comparison of overground and treadmill walking in healthy elderly subjects. Clin. Biomech. 2010, 25, 444-449.

[6] Hustedt, J.W.; Blizzard, D.J.; Baumgaertner, M.R.; Leslie, M.P.; Grauer, J.N. Current advances in training orthopaedic patients to comply with partial weight-bearing instructions. Yale J. Biol. Med. 2012, 85, 119-125.

[7] Isakov, E. Gait rehabilitation: a new biofeedback device for monitoring and enhancing weight-bearing over the affected lower limb. Eura. Medicophys. 2007, 43, 21-26.

[8] Barnett, S.; Cunningham, J.L.; West, S. A comparison of vertical force and temporal parameters produced by an in-shoe pressure measuring system and a force platform. Clin. Biomech. 2001, 16, 353-357.

[9] Bamberg, S.J.M.; Benbasat, A.Y.; Scarborough, D.M.; Krebs, D.E.; Paradiso, J.A. Gait analysis using a shoeintegrated wireless sensor system. IEEE Trans. Inf. Technol. Biomed. 2008, 12, 413-423.

[10] Catalfamo, P.; Moser, D.; Ghoussayni, S.; Ewins, D. Detection of gait events using an F-Scan in-shoe pressure measurement system. Gait Posture 2008, 28, 420-426.

[11] Kernozek, T.W.; LaMott, E.E.; Dancisak, M.J. Reliability of an in-shoe pressure measurement system during treadmill walking. Foot Ankle Int. 1996, 17, 204-209.

[12] Winstein, C.J.; Pohl, P.S.; Cardinale, C.; Green, A.; Scholtz, L.; Waters, C.S. Learning a partial-weight-bearing skill: Effectiveness of two forms of feedback. Phys. Ther. 1996, 76, 985-993.

[13] Hershko, E.; Tauber, C.; Carmeli, E. Biofeedback Versus Physiotherapy in Patients With Partial WeightBearing. Am. J. Orthop. 2008, 37, 92-96.

[14] Bakker, A.; Blokhuis, T.J.; Meeks, M.D. M.E.; Hermens, H.J.; Holtslag, H.R. Dynamic weight loading in older people with hip fracture. J. Rehabil. Med. 2014, 46, 708-711.

[15] Lee, N.K.S.; Goonetilleke, R.S.; Cheung, Y.S.; So, G.M.Y. A flexible encapsulated MEMS pressure sensor system for biomechanical applications. Microsyst. Technol. 2001, 7, 55-62.

[16] Takahashi, K.Z.; Gross, M.T.; Van Werkhoven, H.; Piazza, S.J.; Sawicki, G.S. Adding Stiffness to the Foot Modulates Soleus Force-Velocity Behaviour during Human Walking. Sci. Rep. 2016, 6, 29870. 


\section{PART}
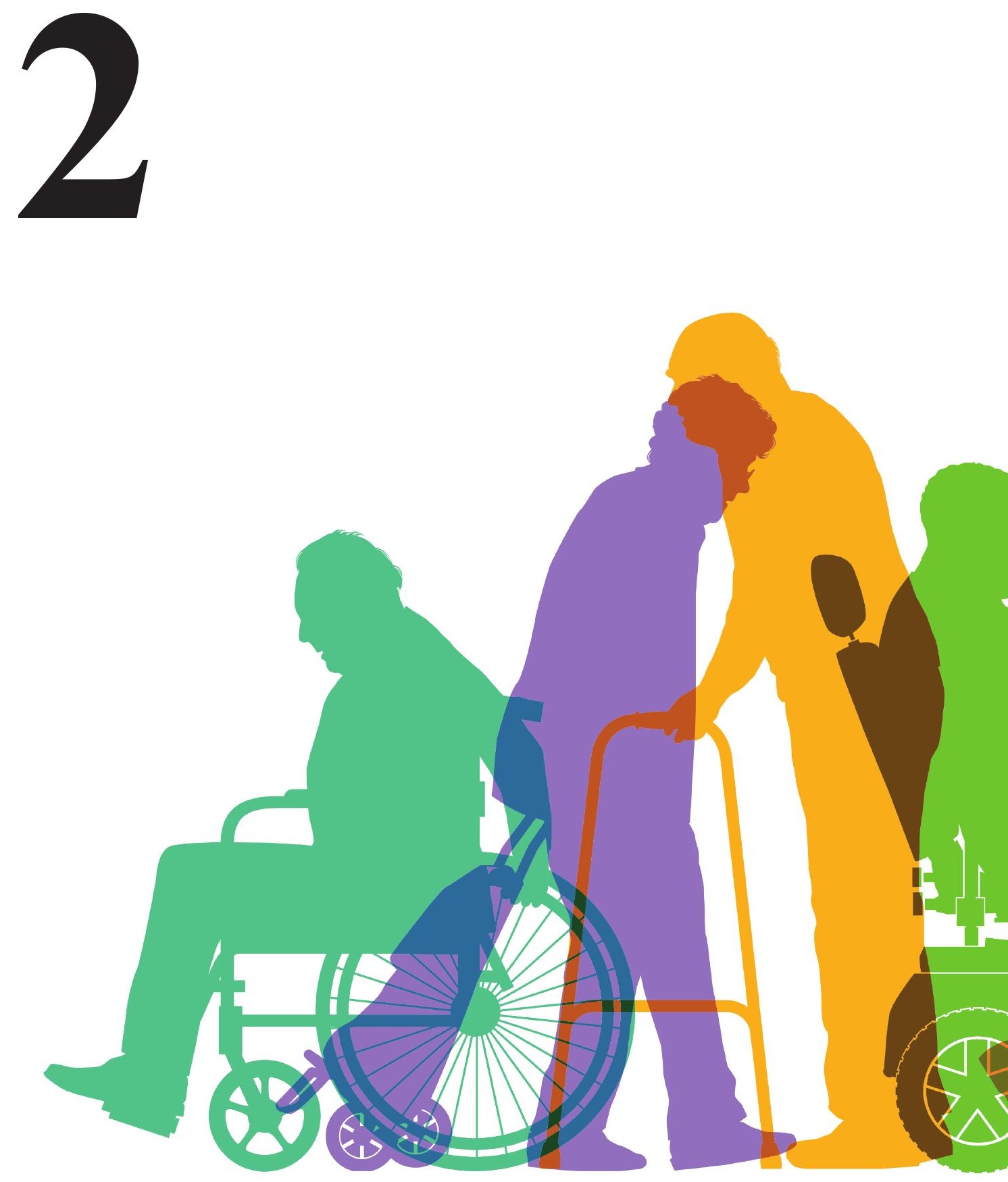


\section{Real-time biofeedback}

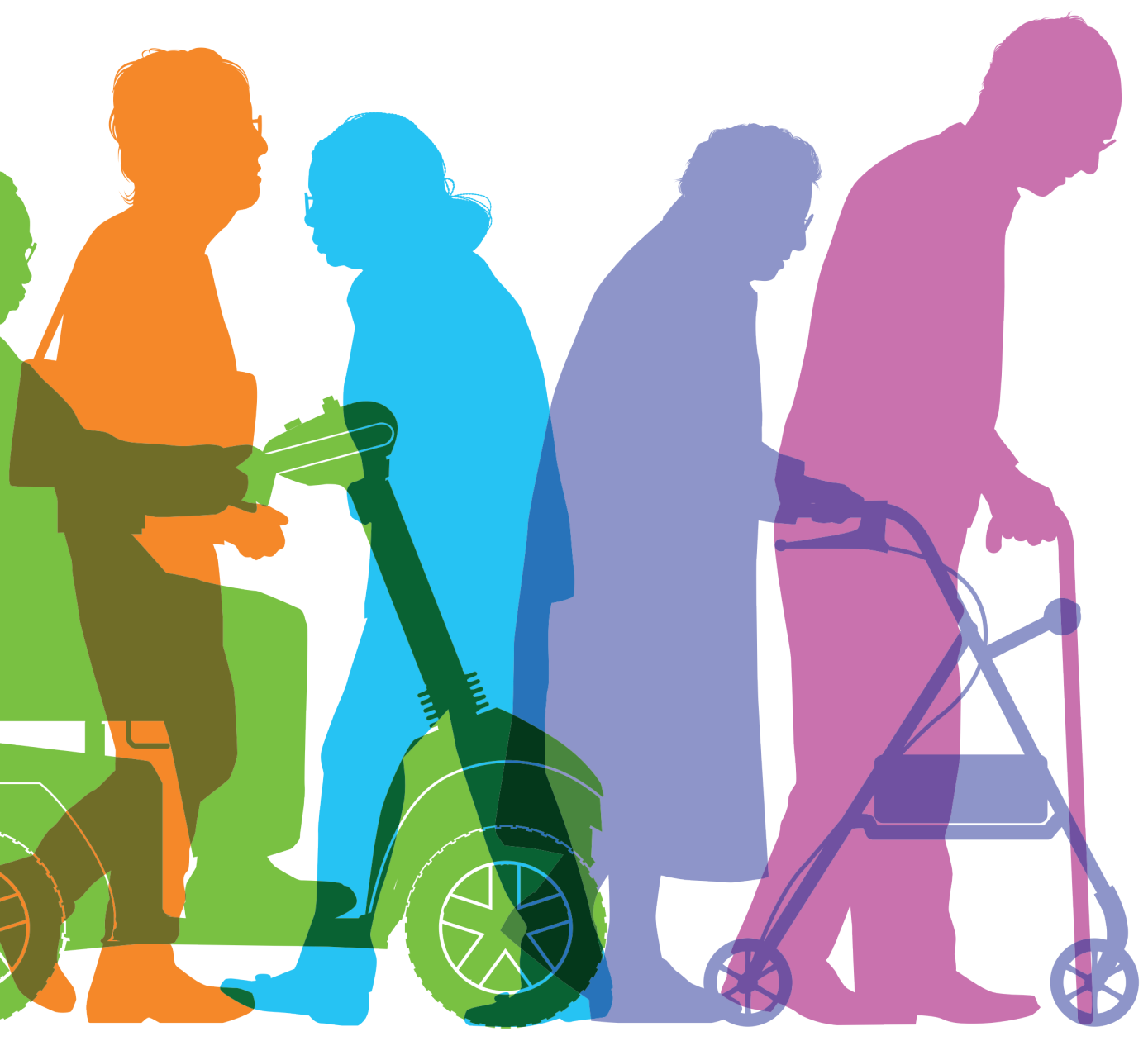


CHAPTER
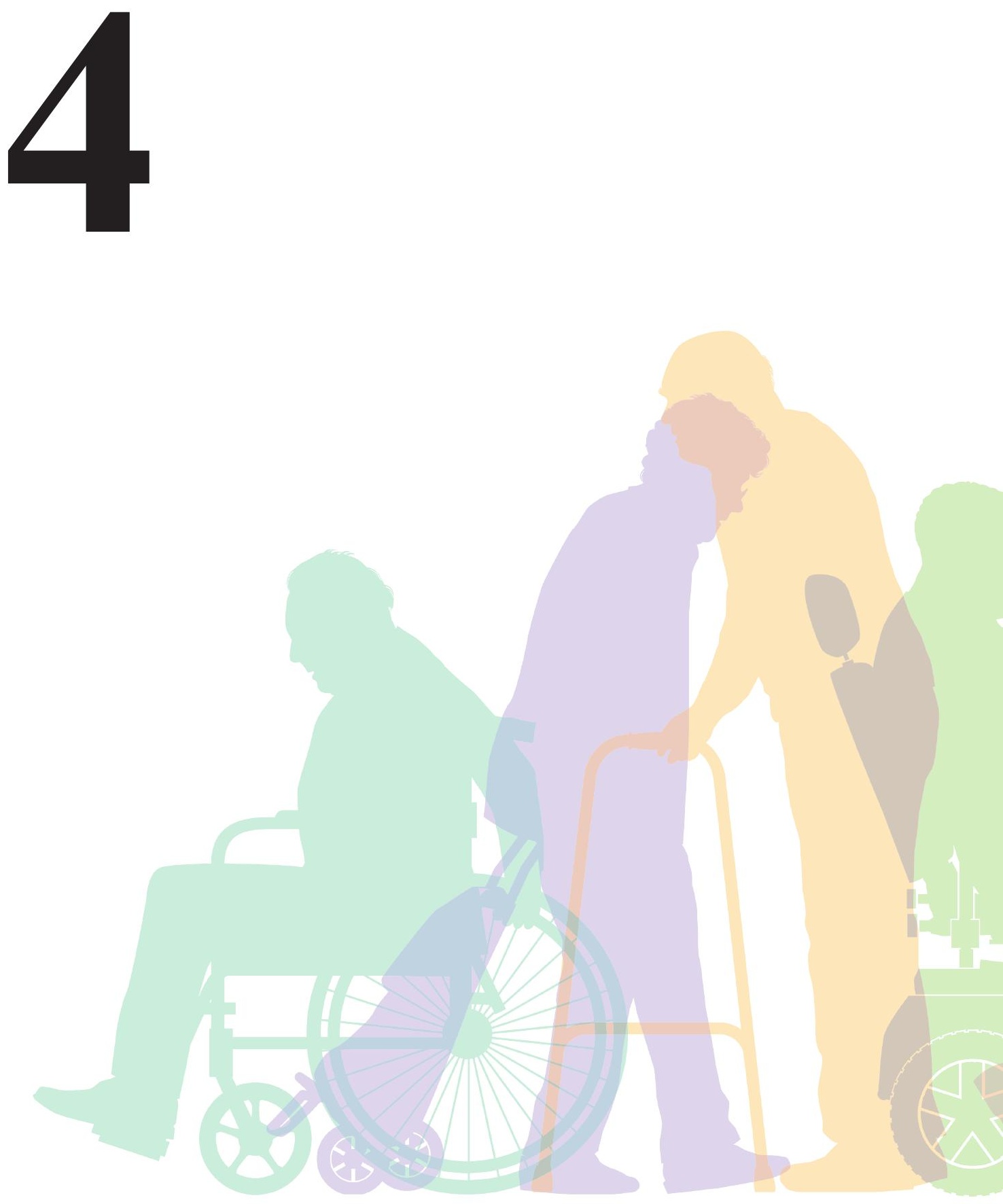
Real-time visual biofeedback during weight bearing improves therapy compliance in patients following lower extremity fractures

Gait \& Posture, January 2018, Volume 59, pp 206-210

M. Raaben, H.R. Holtslag, L.P.H. Leenen, R. Augustine, T.J. Blokhuis 


\begin{abstract}
Background: Individuals with lower extremity fractures are often instructed on how much weight to bear on the affected extremity. Previous studies have shown limited therapy compliance in weight bearing during rehabilitation. In this study we investigated the effect of real-time visual biofeedback on weight bearing in individuals with lower extremity fractures in two conditions: full weight bearing and touch-down weight bearing.
\end{abstract}

Materials and Methods: 11 participants with full weight bearing and 12 participants with touch-down weight bearing after lower extremity fractures have been measured with an ambulatory biofeedback system. The participants first walked 15 meters and the biofeedback system was only used to register the weight bearing. The same protocol was then repeated with real-time visual feedback during weight bearing. The participants could thereby adapt their loading to the desired level and improve therapy compliance.

Results: In participants with full weight bearing, real-time visual biofeedback resulted in a significant increase in loading from $50.9 \pm 7.51 \%$ bodyweight $(\mathrm{BW})$ without feedback to $63.2 \pm 6.74 \% \mathrm{BW}$ with feedback $(\mathrm{P}=0.0016)$. In participants with touch-down weight bearing, the exerted lower extremity load decreased from $16.7 \pm 9.77 \mathrm{~kg}$ without feedback to $10.27 \pm 4.56 \mathrm{~kg}$ with feedback $(\mathrm{P}=0.0718)$. More important, the variance between individual steps significantly decreased after feedback $(\mathrm{P}=0.018)$.

Conclusions: Ambulatory monitoring weight bearing after lower extremity fractures showed that therapy compliance is low, both in full and touch-down weight bearing. Realtime visual biofeedback resulted in significantly higher peak loads in full weight bearing and increased accuracy of individual steps in touch-down weight bearing. Real-time visual biofeedback therefore results in improved therapy compliance after lower extremity fractures.

Keywords: SensiStep, gait monitoring, biofeedback, lower extremity, weight-bearing. 


\section{Introduction}

The weight bearing regime after surgery of lower extremity fractures is controversial and mainly experience based instead of evidence based [1]. Early weight bearing is preferable, as it has beneficial effects on fracture healing. It causes micromovement at the fracture site, which triggers a cascade of cellular events, resulting in the process known as mechanotransduction [2-4]. Furthermore, weight bearing increases bone metabolism and has a positive effect on muscle mass and bone mass [4]. Despite the beneficial effects of early weight bearing, it is avoided in many indications for reasons of safety, for example to protect the mechanical construct or maintain fracture reduction [1]. However, unloading an extremity induces negative effects, such as skeletal muscle atrophy due to decreased transient receptor potential canonical type 1 (TRCP1) protein [5] and loss of bone mineral density $[4,6]$. The treating physician has to determine the optimal weight bearing regime, while balancing between increasing bone growth and muscle mass and protecting the mechanical construct at the fracture site.

Visual biofeedback could improve weight bearing compliance during gait training [7-9]. From previous studies it is known that, in the absence of biofeedback, individuals often experience difficulties in following the prescribed weight bearing regime. This results in limited therapy compliance in full weight bearing, partial weight bearing and touch-down weight bearing [10-14]. In full weight bearing, individuals often underload the fractured extremity due to factors such as pain, anxiety or uncertainty. This could negatively affect the process of mechanotransduction and cause a delay in fracture healing. For partial weight bearing, low therapy compliance results in potentially unsafe conditions by overloading the affected extremity, possibly inducing fracture displacement or mechanical failure. Others have already shown that variance in loading in partial weight bearing is wide, resulting in higher peak loads than anticipated $[10,14,15]$.

The positive effects of biofeedback were previously shown in healthy volunteers using haptic feedback [14] and in individuals with lower extremity injuries using auditory feedback [16,17]. Visual feedback was shown to be effective in Parkinson's disease, in the late period after stroke and after cerebral palsy [7-9]. Still, evidence on effectiveness of biofeedback systems in weight bearing is limited and currently available systems have significant drawbacks, either technological or functional. Many systems are not capable of providing real-time feedback, have not been validated for correct detection of loading, or 
are not available outside a laboratory setting [18]. The SensiStep (Figure 1, Evalan BV, Amsterdam, The Netherlands) has previously been validated in static and dynamic situations and is able to provide real-time visual feedback in the ambulatory setting [19].

The SensiStep was used to investigate the effect of real-time visual biofeedback on weight bearing in the ambulatory, clinical setting in two conditions: full weight bearing and touchdown weight bearing. We hypothesize that therapy compliance can be improved using visual biofeedback in individuals after lower extremity fractures. Ideally this should result in higher peak loads during full weight bearing, and decreased variances in peak loads during touch-down weight bearing.

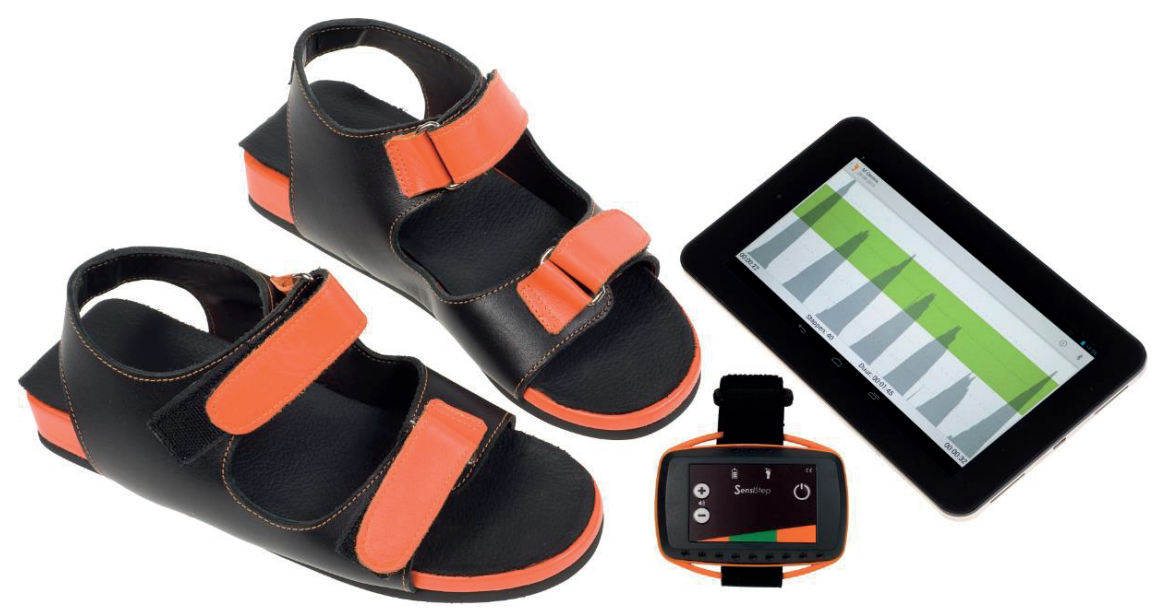

Figure 1. The SensiStep system. Real-time visual biofeedback was shown on the tablet as a green bar (i.e. target weight) with grey step curves (actual weight). Both the participant and the physical therapist had insight in weight bearing during the feedback session.

\section{Materials and methods}

\section{Inclusion and exclusion criteria}

In a level I trauma center, individuals who underwent surgery of the lower extremity after trauma were asked to participate. Surgery was either performed within days following a fracture (fresh fractures), or late to treat long term complications, e.g. a non-union or a malunion (revision surgery). The participants were allowed either full weight bearing or touch-down weight bearing following the surgical procedure at the discretion of the treating physician. Exclusion criteria were 1) weight $>120 \mathrm{~kg}$, 2) cognitive impairment or 3) inability to achieve dorsiflexion of the ankle joint, as the position of the sensor in the 
SensiStep system requires a neutral foot posture. This research protocol was approved by the medical ethics review board of the institution (approval number 11-317/C).

\section{Study design}

The participants received either conservative treatment or underwent surgical procedures for fixation of a lower extremity fracture or revision of previous posttraumatic injuries. The first day of conservative treatment or after the surgical procedure, participants started mobility exercises under direct supervision of an experienced physical therapist according to standard institutional protocol. This protocol included training exercises to walk safely, if needed with the use of walking aids. All physical therapists involved in this study were qualified for orthopedic and trauma rehabilitation. They were appointed solely to these departments. The standard institutional protocol for mobilizing the participants was repeated daily. The study protocol started at the day the participants were able to walk safely, as determined by the physical therapist. The participants were instructed to walk 15 meters in a straight line with SensiStep without feedback, meaning that neither the participant nor the physical therapist had direct insight in weight bearing. Immediately thereafter, in a return walk, the participant and the physical therapist received real-time visual feedback on loading, with the desired level of loading indicated on the tablet. The order of walking without feedback followed by walking with feedback was deliberately chosen to avoid the potential influence of a learning effect. Previous studies are inconsistent about the learning effect of biofeedback and an immediate effect cannot be ruled out [20]. Feedback was provided using a tablet with graphic real-time representation of each step. The applied weight of individual steps was directly visualized, as well as the desired target weight, which was illustrated as a green bar in the same graph (Figure 1). The healthcare professional, also looking at the tablet, verbally assisted and motivated the participant to improve weight bearing. For participants with full weight bearing, the target load was set at $100 \%$ bodyweight (BW) (range: $\pm 10 \mathrm{~kg}$ ). For participants with touch-down weight bearing, the target load was set at 10 kilograms $(\mathrm{kg})$ (range: $\pm 5.0 \mathrm{~kg}$ ), as previously defined by others $[10,12,14]$.

\section{Data analysis}

All raw data were encrypted and stored on a secured server. The raw data were analyzed with MATLAB 2014a. Data from full weight bearing sessions were analyzed separately from data from touch-down weight bearing sessions. Specific Matlab routines were 
developed to convert the raw data into the parameters of interest, the peak load and the loading rate. The peak load of a single step was previously defined as the maximum peak during the entire gait cycle of a step [21]. Per participant, the mean peak load per session was calculated by taking the average peak load of all single steps. The results are shown in percentage bodyweight $(\% \mathrm{BW})$ for full weight bearing and in kilograms $(\mathrm{kg})$ for touchdown weight bearing. The loading rate was defined as the steepness of the curve and shown for both weight bearing regimes in kilograms per millisecond $(\mathrm{kg} / \mathrm{ms})$. The mean loading rate per session was used for statistical analysis. Both parameters have previously shown a clear relation with rehabilitation progress in elderly with hip fractures [21]. It is expected that both peak load and loading rate will increase when the participants reach the endpoint of rehabilitation [21].

\section{Statistical analysis}

The D'Agostino-Pearson omnibus test was used to confirm normal distribution of the datasets. Then, the paired Student's T-test in Prism 7.02 (GraphPad Software Inc., La Jolla, CA, USA) was used to determine differences in weight bearing parameters between the training with and without feedback from SensiStep. To compare variance of the data, specifically in touch-down weight bearing, the F-test was used. All data are shown as mean \pm standard deviation (SD). Significance was set at $\alpha<0.05$.

\section{Results}

Between August 2015 and February 2016, a total of twenty-six consecutive individuals were eligible for inclusion. Three of these were excluded; one was unable to achieve dorsiflexion of the ankle joint and in two cases technical failure of the tablet occurred. Eleven participants ( 7 male, 4 female) with a mean age of 43.7 years (range: 19.0 years 83.0 years) and average weight of $81.5 \mathrm{~kg}$ (range: $58.0 \mathrm{~kg}-115.0 \mathrm{~kg}$ ) were allowed full weight bearing. Of these participants, six were treated for fresh fractures and five underwent revision surgery. Twelve participants ( 9 male, 3 female) with a mean age of 49.1 years (range: 26.0 years -79.0 years) and an average weight of 77.8 kilogram (range: 58.0 $\mathrm{kg}-100.0 \mathrm{~kg}$ ) were allowed touch-down weight bearing. All participants who were allowed touch-down weight bearing had fresh fractures. Demographic data are shown in table 1 . The measurements were performed on average at day five after surgery (range: $1-$ 11 days). 


\begin{tabular}{|c|c|c|}
\hline & Full weight bearing & Touch-down weight bearing \\
\hline Number of participants & $11(7 \hat{\sigma}-4$ 우 $)$ & $12(9 \hat{\circ}-3+)$ \\
\hline Mean age (range) & 43.7 years $(19.0-83.0)$ & 49.1 years $(26.0-79.0)$ \\
\hline Mean weight (range) & $81.5 \mathrm{~kg}(58.0-115.0)$ & $77.8 \mathrm{~kg}(58.0-100.0)$ \\
\hline \multicolumn{3}{|l|}{ Type of injury } \\
\hline Fresh fracture & 6 & 12 \\
\hline Revision & 5 & 0 \\
\hline \multicolumn{3}{|l|}{ Fracture side } \\
\hline Left & 8 & 9 \\
\hline Right & 3 & 3 \\
\hline \multicolumn{3}{|l|}{ Type of treatment } \\
\hline Conservative & 4 & 5 \\
\hline Surgery & 7 & 7 \\
\hline
\end{tabular}

Table 1. Demographics of participants with full weight bearing and touch-down weight bearing (mean value and range). All participants underwent conservative or surgical treatment after lower extremity fractures.

Full weight bearing without feedback resulted in limited peak loads, both after a fresh fracture as well as after revision surgery. Despite standard guidance and instructions by experienced physical therapists, the mean peak load in participants with full weight bearing was only $50.9 \pm 7.51 \% \mathrm{BW}$. For participants with fresh fractures and participants after revision surgery, the mean peak load was respectively $55.6 \pm 10.30 \% \mathrm{BW}$ and $47.0 \pm 5.20$ \%BW $(\mathrm{P}=0.6112)$. Real-time feedback by SensiStep resulted in a significant increase in peak load to $63.2 \pm 6.74 \% \mathrm{BW}(\mathrm{P}=0.0016$, figure $2 \mathrm{a})$. Separate analysis of participants with fresh fractures resulted in a significant higher peak load of $65.4 \pm 7.75 \% \mathrm{BW}$ after feedback $(\mathrm{P}=0.0426)$. In participants with revision surgery, the peak load with real-time feedback was significantly increased to $61.4 \pm 5.89 \% \mathrm{BW}(\mathrm{P}=0.0252)$. The variance in participants who were allowed full weight bearing did not change significantly.

The loading rate was $0.045 \pm 0.014 \mathrm{~kg} / \mathrm{ms}$ without feedback for the full weight bearing participants. A significant increase in loading rate was found after feedback: $0.059 \pm 0.020$ $\mathrm{kg} / \mathrm{ms}(\mathrm{P}=0.0479$, figure $2 \mathrm{~b})$. Loading rate was similar for participants with fresh fractures and participants after revision surgery, respectively $0.032 \pm 0.008 \mathrm{~kg} / \mathrm{ms}$ and $0.056 \pm 0.020$ $\mathrm{kg} / \mathrm{ms}(\mathrm{P}=0.4511)$. Feedback did not change these values significantly: $0.043 \pm 0.014 \mathrm{~kg} / \mathrm{ms}$ 
for participants with fresh fractures $(\mathrm{P}=0.0628)$ and $0.073 \pm 0.025 \mathrm{~kg} / \mathrm{ms}$ for participants after revision surgery $(\mathrm{P}=0.2152)$.

All participants who were allowed touch-down weight bearing were treated for fresh fractures. Without feedback the mean peak load was $16.7 \pm 9.77 \mathrm{~kg}$. The range in peak load, which is considered important in safety, was $2.0-42.0 \mathrm{~kg}$. The mean peak load after feedback decreased to $10.3 \pm 4.56 \mathrm{~kg}$ ( $\mathrm{ns}, \mathrm{P}=0.0718$ ). More importantly, the variance significantly decreased $(\mathrm{P}=0.018)$ and the maximum peak load detected was $25 \mathrm{~kg}$ (range: $2.0-25.0$, figure $3 \mathrm{a}$ ). The loading rate in the touch-down weight bearing group was 0.026 $\pm 0.015 \mathrm{~kg} / \mathrm{ms}$. No significant difference in loading rate was seen when feedback was given: $0.022 \pm 0.014 \mathrm{~kg} / \mathrm{ms}(\mathrm{P}=0.2124$, figure $3 \mathrm{~b})$.

\section{Discussion}

There is little consensus on the weight bearing regime that should be prescribed after surgery of the lower extremities. Depending on the type of mechanical construct, bone quality, fracture site, or the background of the treating physician, strict weight bearing regimes are often prescribed, ranging from non-weight bearing, touch-down weight bearing, partial weight bearing to full weight bearing [1]. As described in previous studies, individuals often experience difficulties to apply correct weight bearing, which results in limited therapy compliance $[10,11,13]$. This study confirms these findings in both full weight bearing and touch-down weight bearing. Moreover, this study also demonstrates the positive influence of real-time visual biofeedback on weight bearing in a clinical setting.

Both touch-down weight bearing and full weight bearing were significantly influenced by real-time biofeedback. For touch-down weight bearing this effect was specifically observed in the variance in peak load. The large variance in peak load, as seen prior to biofeedback, resulted in peak loads well above the prescribed 10 kilograms, up to a maximum of $42 \mathrm{~kg}$. These high peak loads cause potentially unsafe conditions, as fracture displacement or mechanical failure could occur. The mean peak load was reduced by implementing realtime visual feedback, but more importantly, the variance in peak load significantly decreased. The maximum peak load, as observed in feedback conditions, was reduced to 25 $\mathrm{kg}$. 
Full weight bearing $(n=11)$

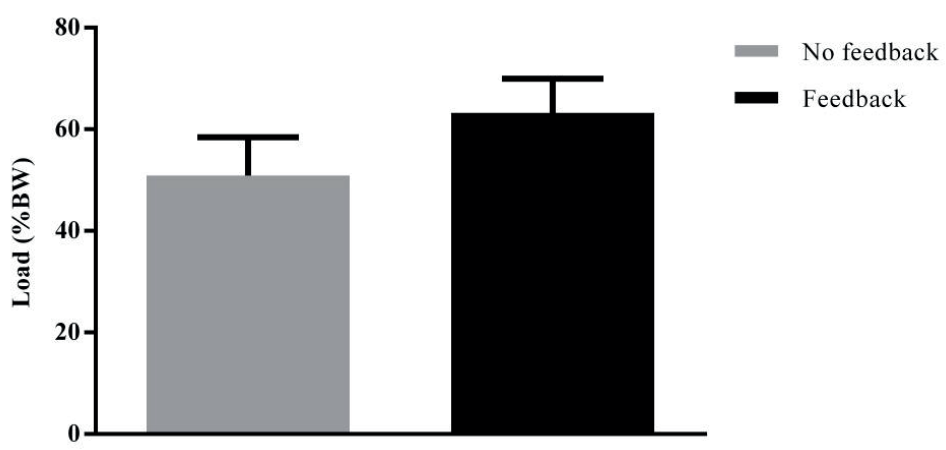

Figure 2a. The average peak load was $50.9 \pm 7.51 \% \mathrm{BW}$ when using standard training methods in participants with full weight bearing. After real-time visual biofeedback a significantly higher average peak load of $63.2 \pm 6.74 \% \mathrm{BW}$ was observed $(\mathrm{P}=0.0016)$.

Full weight bearing $(n=11)$

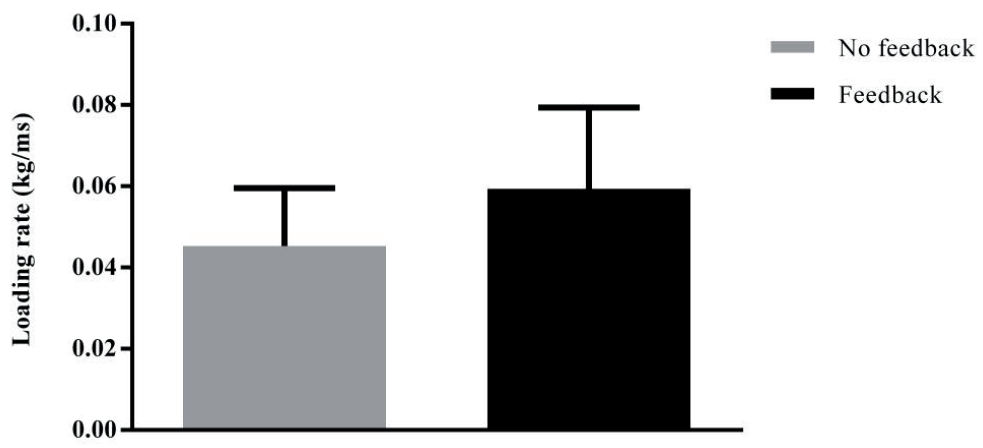

Figure $2 \mathrm{~b}$. A significant increase in loading rate was found after providing real-time visual feedback to participants with full weight bearing. The loading rate was $0.045 \pm 0.014 \mathrm{~kg} / \mathrm{ms}$ when using standard training methods and $0.059 \pm 0.020$ $\mathrm{kg} / \mathrm{ms}$ with real-time visual biofeedback $(\mathrm{P}=0.0479)$. 
Touch down weight bearing $(\mathrm{n}=12)$

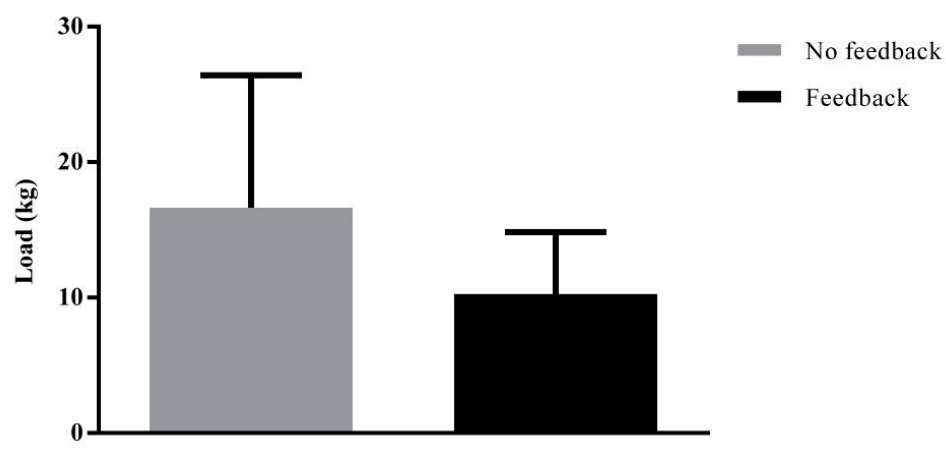

Figure 3a. Participants with touch-down weight bearing showed an average peak load of $16.7 \pm 9.77 \mathrm{~kg}$ when using standard training methods. Real-time visual biofeedback resulted in an average peak load of $10.27 \pm 4.56 \mathrm{~kg}$. A significant decrease in the variance in peak load was found, as illustrated by the standard deviation $(\mathrm{P}=0.018)$.

Touch down weight bearing $(\mathrm{n}=12)$

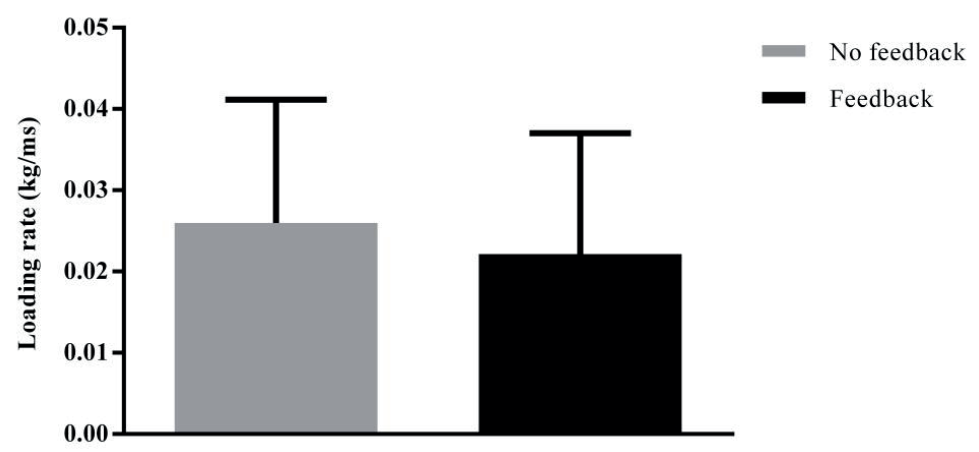

Figure 3b. No significant differences in loading rate were found in participants with touch-down weight bearing: $0.026 \pm$ $0.015 \mathrm{~kg} / \mathrm{ms}$ with standard training methods and $0.022 \pm 0.014 \mathrm{~kg} / \mathrm{ms}$ after real-time visual biofeedback. 
The current study also indicates that the participants are not able to implement full weight bearing, despite the standard training methods provided by experienced physical therapists. Although achieving $100 \% \mathrm{BW}$ in the full weight bearing participants was difficult due to the use of walking aids, the data clearly indicated that full weight bearing was improved by real-time visual biofeedback, both in participants with fresh fractures and after revision surgery. In addition, a clear difference in gait parameters was observed between participants with fresh fractures and participants after revision surgery. Participants after revision surgery had lower initial peak loads. This indicates that participants after revision surgery, despite earlier training, were reluctant to bear weight on the affected extremity. This underlines the need for biofeedback systems to ensure high quality rehabilitation after lower extremity surgery, in particular after revision surgery.

The findings of the current study are in line with previous studies in healthy volunteers [15] and in persons with lower extremity fractures [10-13]. These studies showed low therapy compliance in partial weight bearing when using standard training methods. Also, previous studies have described the potential of using biofeedback systems to improve weight bearing. Although the beneficial effects of biofeedback are recognized by many, the ideal way to provide feedback is still unknown. Haptic feedback has shown good results for touch-down weight bearing in healthy volunteers [14], whereas others have shown improved therapy compliance in individuals with partial weight bearing using auditory biofeedback [16]. However, the use of auditory feedback is not ideal as persons continue to load after an alarm is activated, known as the overshoot phenomenon, caused by a physiologic response time of 150 to 250 milliseconds [16]. This could lead to potentially harmful situations by overloading the fracture site. As shown by the data in the current study, this overshoot effect is not evident when using real-time visual feedback, possibly because participants get visual confirmation of each step real-time, enabling them to anticipate when the target load is nearly achieved.

Comparing the results of the parameter loading rate to other studies is hampered by the use of different techniques and definitions. In the current study, biofeedback yielded no significant effect on loading rate in participants with touch-down weight bearing. A possible explanation for this result is the fact that all participants in this group were recovering from fresh fractures. As described previously [21], the loading rate reflects a mechanism that is influenced by pain, anxiety or confidence. Participants with fresh 
fractures recently underwent a painful and stressful event, which ultimately resulted in the lower extremity fracture. As indicated by previous studies, factors as pain and fear of falling have a negative impact on gait and rehabilitation [22,23], which apparently is not easily resolved by visual feedback during a short period of time. This hypothesis is supported by the findings in full weight bearing, where higher loading rates were seen after revision surgery. Persons undergoing revision surgery may have less anxiety, or more confidence, in the early phase after surgery. A second possible explanation for the lack of improvement in loading rate could be related to the method of feedback. Instead of providing feedback on loading rate, the feedback was given on the peak load. The participants were therefore focused on improving peak load instead of the loading rate, resulting in slower steps and consequently in lower loading rates. Possibly, specific visual feedback on loading rate may improve this parameter in the early post-operative phase.

This study is the first to demonstrate the effect of real-time visual biofeedback in individuals with touch-down and full weight bearing, shortly after treatment for lower extremity fractures in the clinical environment. However, in interpretation of the data shown, several limitations have to be kept in mind. First, the measurements were obtained only during hospital admission of each participant. Therefore, changes over a longer period of time or during progress of rehabilitation were not monitored. This leaves questions on the retention and learning effect of biofeedback unanswered for now. No follow up was chosen in the study design, as the primary aim of this study was to gain insight in the direct effects of real-time visual biofeedback on weight bearing during the early phase of rehabilitation. Second, the clinical relevance of the finding that weight bearing can be improved by real-time biofeedback is yet to be determined. Although it is suggested that mechanotransduction improves fracture healing, clinical evidence to support this claim is limited. Still, the need for biofeedback to improve loading and gait is expressed by many. Third, the number of participants per group is relatively small. Still, even in this relatively small population several significant changes were observed. One of the strengths is the cross-over study design, whereby each participant was exposed to both the standard treatment (no biofeedback) and the intervention (biofeedback). Although this method prohibits a follow-up, as participants have already been exposed to the intervention after one session, it excludes potential bias based on different population characteristics. Second, by including both fresh fractures and revision surgery participants, heterogeneity of the study group was introduced. Moreover, including participants with full weight bearing and 
touch-down weight bearing provided insight in the effect in these two different conditions as well. For all these subgroups the effect of real-time biofeedback was clear.

\section{Conclusion}

Standard training methods lead to limited therapy compliance in weight bearing after fractures of the lower extremities. Real-time visual biofeedback on weight bearing significantly improves therapy compliance in two conditions: touch-down weight bearing and full weight bearing. Although a larger, multicenter study is needed, the use of biofeedback systems may be beneficial in future rehabilitation programs. 


\section{References}

[1] E.N. Kubiak, M.J. Beebe, K. North, R. Hitchcock, M.Q. Potter, Early weight bearing after lower extremity fractures in adults., J. Am. Acad. Orthop. Surg. 21 (2013) 727-38. doi:10.5435/JAAOS-21-12-727.

[2] J. Klein-Nulend, R.G. Bacabac, M.G. Mullender, Mechanobiology of bone tissue, Pathol. Biol. 53 (2005) $576-$ 580. doi:10.1016/j.patbio.2004.12.005.

[3] S.G. Childs, Stimulators of Bone Healing, Orthop. Nurs. 22 (2003) 421-428.

[4] A.G. Robling, A.B. Castillo, C.H. Turner, Biomechanical and Molecular Regulation of Bone Remodeling, Annu. Rev. Biomed. Eng. 8 (2006) 455-98. doi:10.1146/annurev.bioeng.8.061505.095721.

[5] L. Xia, K.-K. Cheung, S.S. Yeung, E.W. Yeung, The involvement of TRPC1 in skeletal muscle regrowth after unloading-induced atrophy, J. Physiol. 0 (2016). doi:10.1113/JP271705.

[6] H.E. Van der Wiel, P. Lips, J. Nauta, P. Patka, H.J.T.M. Haarman, G.J.J. Teule, Loss of Bone following in the Proximal Part of the Femur following Unstable Fractures of the Leg, J. Bone Jt. Surg. 76-A (1994) 230-236.

[7] Y. Jiang, K.E. Norman, Effects of visual and auditory cues on gait initiation in people with Parkinson's disease, Clin. Rehabil. 20 (2006) 36-45. doi:10.1191/0269215506cr925oa.

[8] M. Druzbicki, A. Guzik, G. Przysada, A. Kwolek, A. Brzozowska-Magoń, Efficacy of gait training using a treadmill with and without visual biofeedback in patients after stroke: A randomized study, J. Rehabil. Med. 47 (2015) 419-425. doi:10.2340/16501977-1949.

[9] Y. Baram, R. Lenger, Gait improvement in patients with cerebral palsy by visual and auditory feedback, Neuromodulation. 15 (2012) 48-52. doi:10.1111/j.1525-1403.2011.00412.x.

[10] F.K. Ruiz, M.C. Fu, D.D. Bohl, J.W. Hustedt, M.R. Baumgaertner, M.P. Leslie, J.N. Grauer, Patient Compliance With Postoperative Lower Extremity Touch-down Weight-bearing Orders at a Level I Academic Trauma Center, Orthopedics. 37 (2014) e552-e556. doi:10.3928/01477447-20140528-55.

[11] A. Vasarhelyi, T. Baumert, C. Fritsch, W. Hopfenmüller, G. Gradl, T. Mittlmeier, Partial weight bearing after surgery for fractures of the lower extremity - is it achievable?, Gait Posture. 23 (2006) 99-105. doi:10.1016/j.gaitpost.2004.12.005.

[12] J.W. Hustedt, D.J. Blizzard, M.R. Baumgaertner, M.P. Leslie, J.N. Grauer, Is it possible to train patients to limit weight bearing on a lower extremity?, Orthopedics. 35 (2012) e31-7. doi:10.3928/01477447-20111122-14.

[13] B.J. Braun, N.T. Veith, M. Rollmann, M. Orth, T. Fritz, S.C. Herath, J.H. Holstein, T. Pohlemann, Weightbearing recommendations after operative fracture treatment-fact or fiction? Gait results with and feasibility of a dynamic, continuous pedobarography insole, Int. Orthop. (2017) 1-6. doi:10.1007/s00264-017-3481-7.

[14] M.C. Fu, L. DeLuke, R. a Buerba, R.E. Fan, Y.J. Zheng, M.P. Leslie, M.R. Baumgaertner, J.N. Grauer, Haptic biofeedback for improving compliance with lower-extremity partial weight bearing., Orthopedics. 37 (2014) e993-8. doi:10.3928/01477447-20141023-56.

[15] S. Li, C.W. Armstrong, D. Cipriani, Three-point gait crutch walking: Variability in ground reaction force during weight bearing, Arch. Phys. Med. Rehabil. 82 (2001) 86-92. doi:10.1053/apmr.2001.16347. 
[16] E. Hershko, C. Tauber, E. Carmeli, Biofeedback Versus Physiotherapy in Patients With Partial Weight-Bearing, Am. J. Orthop. 37 (2008) 92-96.

[17] E. Isakov, Gait rehabilitation: a new biofeedback device for monitoring and enhancing weight-bearing over the affected lower limb, Eura. Medicophys. 43 (2007) 21-26.

[18] H.L.P. Hurkmans, J.B.J. Bussmann, E. Benda, J. a N. Verhaar, H.J. Stam, Techniques for measuring weight bearing during standing and walking, Clin. Biomech. 18 (2003) 576-589. doi:10.1016/S0268-0033(03)00116-5.

[19] M. Raaben, H. Holtslag, R. Augustine, R. van Merkerk, B. Koopman, T. Blokhuis, Technical Aspects and Validation of a New Biofeedback System for Measuring Lower Limb Loading in the Dynamic Situation, Sensors. 17 (2017) 658. doi:10.3390/s17030658.

[20] J.W. Hustedt, D.J. Blizzard, M.R. Baumgaertner, M.P. Leslie, J.N. Grauer, Lower-extremity Weight-bearing Compliance Is Maintained Over Time After Biofeedback Training, Orthopedics. 35 (2012) e1644-e1648. doi:10.3928/01477447-20121023-24.

[21] A. Bakker, T.J. Blokhuis, M.D.M.E. Meeks, H.J. Hermens, H.R. Holtslag, Dynamic weight loading in older people with hip fracture., J. Rehabil. Med. (2014) 1-4. doi:10.2340/16501977-1793.

[22] R.C. Oude Voshaar, S. Banerjee, M. Horan, R. Baldwin, N. Pendleton, R. Proctor, N. Tarrier, Y. Woodward, A. Burns, Fear of falling more important than pain and depression for functional recovery after surgery for hip fracture in older people, Psychol. Med. 36 (2006) 1635. doi:10.1017/S0033291706008270.

[23] R.S. Morrison, J. Magaziner, M.A. McLaughlin, G. Orosz, S.B. Silberzweig, K.J. Koval, A.L. Siu, The impact of post-operative pain on outcomes following hip fracture, Pain. 103 (2003) 303-311. doi:10.1016/S0304-3959(02)00458$\mathrm{X}$. 
CHAPTER
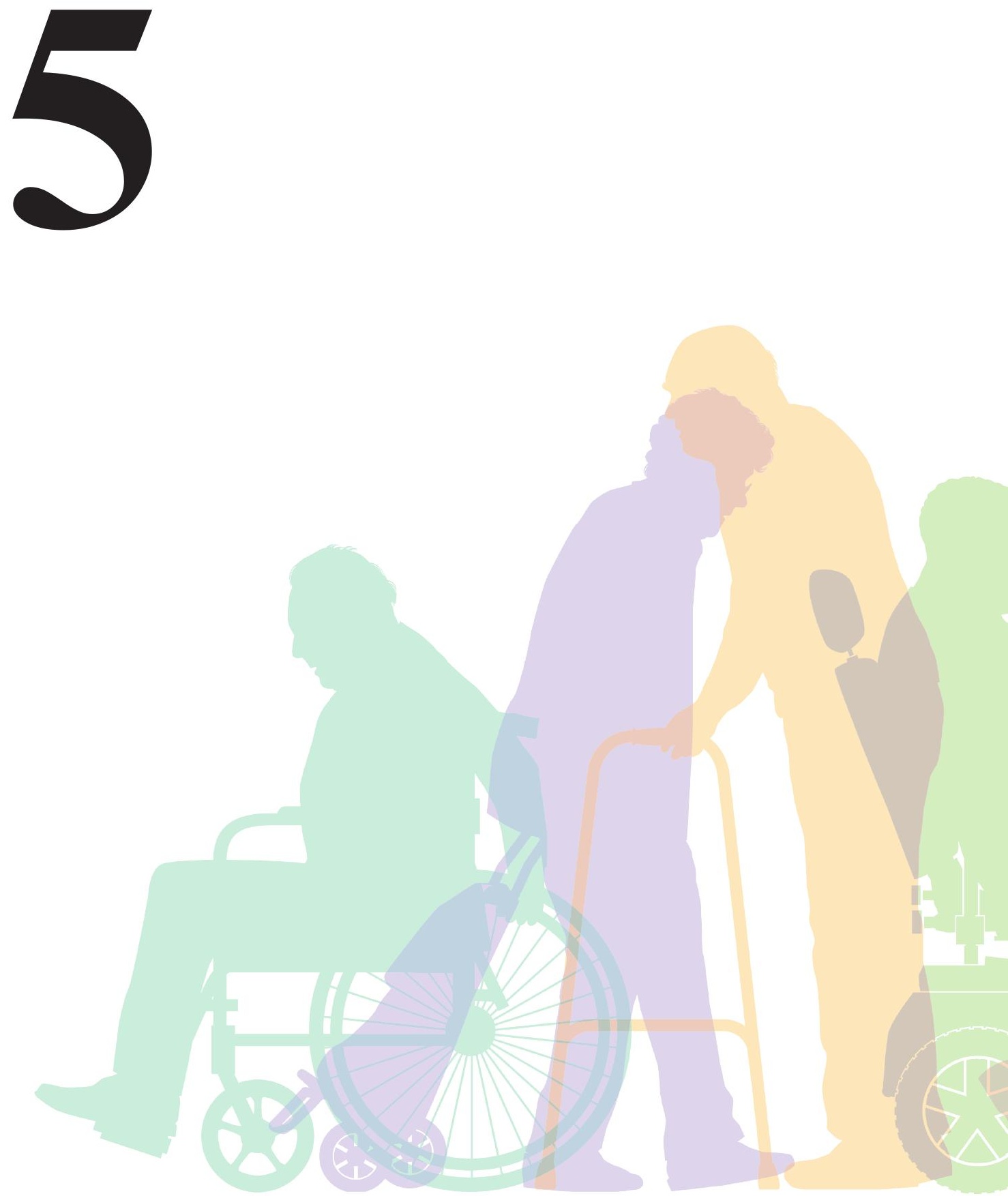
Real-time visual biofeedback to improve therapy compliance after total hip arthroplasty: A pilot randomized controlled trial

Gait \& Posture, March 2018, Volume 61, pp 306-310

M. Raaben, H.C. Vogely, T.J. Blokhuis 


\begin{abstract}
Background: Previous studies have shown limited therapy compliance in weight-bearing in patients following total hip arthroplasty. The purpose of this pilot RCT is to determine the immediate and late effect of real-time, visual biofeedback on weight-bearing during rehabilitation after THA in elderly.

Materials and Methods: 24 participants who underwent THA were randomized to either the control or the intervention group. The intervention group received real-time, visual biofeedback on weight-bearing during training with the physical therapist during hospitalization and at twelve weeks follow up.

Results: Without biofeedback, therapy compliance was limited. Significant improvement in peak load was found in the intervention group in the early postoperative phase. In contrast to the control group, the peak load at twelve weeks was significantly higher in the intervention group compared to the pre-operative peak load, indicating a lasting effect of early biofeedback. Other gait parameters were not significantly different in the early postoperative phase. In the intervention group a longer walking distance was observed and the use of walking aids was reduced at twelve weeks.
\end{abstract}

Conclusions: Biofeedback systems could be promising to improve outcomes and reduce costs in future rehabilitation programs after THA.

Keywords: SensiStep, gait monitoring, biofeedback, rehabilitation, total hip arthroplasty. 


\section{Introduction}

Total hip arthroplasty (THA) is a common and effective procedure in the treatment of coxarthrosis [1,2]. The demand for this procedure is still growing due to the ageing population worldwide [3]. To optimise the treatment and to decrease growing healthcare costs, many improvements have been made in surgical techniques to ensure rapid recovery in the early postoperative phase [4]. Although a meticulous surgical technique for THA is important, rapid recovery also depends on interventions performed in the early postoperative phase [5]. Early, full weight-bearing has positive effects on general coordination and muscle strength $[5,6]$ and prevents complications, such as osteoporosis, bedsores and pneumonia [7]. Previous studies have already shown that early, full weightbearing is safe and does not increase the incidence of postoperative complications [5,7]. The absence of an evidence-based, worldwide guideline on rehabilitation after THA is, in this light, remarkable.

In addition, currently used protocols and technologies during rehabilitation are outdated or of limited value. Clinical mobility scores are commonly used to observe the progress of individuals in a rehabilitation setting. The most frequently used Harris Hip Score, however, possesses ceiling effects and has potential clinician bias [8]. Moreover, using a score hardly offers the opportunity to give adequate feedback to the patient or the physician, which makes it difficult to improve gait. Bathroom scales and vocal instructions are often used as guiding techniques to improve weight-bearing, but their information is lost in the dynamic situation. This leads to limited therapy compliance, as previously shown in both healthy volunteers and patients [9-12]. Biofeedback systems offer the opportunity to measure weight-bearing in the dynamic situation [13]. In this context, the use of biofeedback systems is promising, especially when real-time feedback is given.

Biofeedback systems were already used to improve gait symmetry after THA $[14,15]$. Although gait symmetry is important, far less studies have been performed on weightbearing of the affected limb. Some studies have reported positive effects if partial weightbearing was allowed [16-18]. However, since early, full weight-bearing has beneficial effects on healing outcome, the aim of this pilot RCT (randomized controlled trial) was to investigate the therapy compliance of patients with unrestricted weight-bearing following THA. In addition, it was determined whether real-time, visual feedback improved full weight-bearing in the early postoperative phase. 


\section{Patients and Methods}

\section{Inclusion and exclusion criteria}

Patients aged between 60 and 85 years who underwent THA for treatment of primary coxarthrosis were eligible for inclusion in this study. Patients with impaired mobility prior to surgery due to causes other than coxarthrosis were excluded, as well as patients with posttraumatic coxarthrosis. Patients with impaired cognition, either pre-existent or postoperative, were also excluded. Informed consent was obtained for all patients. This research protocol was approved by the local Ethics Committee (approval number 14-559/D) of our institution, a university medical center with full orthopaedic facilities, including a resident program.

\section{Operative procedure}

All participants underwent THA by a senior orthopaedic surgeon. The systems used were an Exceed/Taperloc $(n=4)$, a Mallory/Taperloc $(n=2)$, an Exceed/Stanmore $(n=14)$ and an Advantage/Stanmore $(n=1)$. A standardized protocol for surgery was followed. In short, the participants were operated in full lateral position, secured with positioning devices to ensure complete stability. After draping and under antibiotic prophylaxis, a lateral $(n=6)$ or posterolateral $(n=18)$ approach was used to visualise and dislocate the hip joint. The femoral head was removed by sawing through the collum femoris. Then the acetabulum was prepared and gentamicin loaded cement was used for fixation of the acetabulum cup. The intramedullary canal of the proximal femur was prepared and gentamicin loaded cement was used for fixation of the steel. Following insertion of the correct size of the head, the hip joint was reduced and stability and function were tested to ensure a proper end result. Post-operatively, all participants were allowed full weight-bearing. Early mobilisation of the participants was ensured by physical therapists dedicated to the orthopaedic ward within $24 \mathrm{~h}$ after surgery.

\section{Biofeedback system}

Weight-bearing was measured in the clinical setting using an ambulatory biofeedback system (SensiStep, Evalan BV, Amsterdam, The Netherlands). In short, SensiStep consists of an in-sole sensor, which registers axial loading on the affected extremity accurately and continuously during gait. In addition to ambulatory force measurements, SensiStep is able to provide real-time, visual feedback about weight-bearing to both the patient and the 
healthcare professional via a bracelet and/or tablet as shown in Figure 1 [19]. The SensiStep system was previously validated in static and dynamic situations [20].

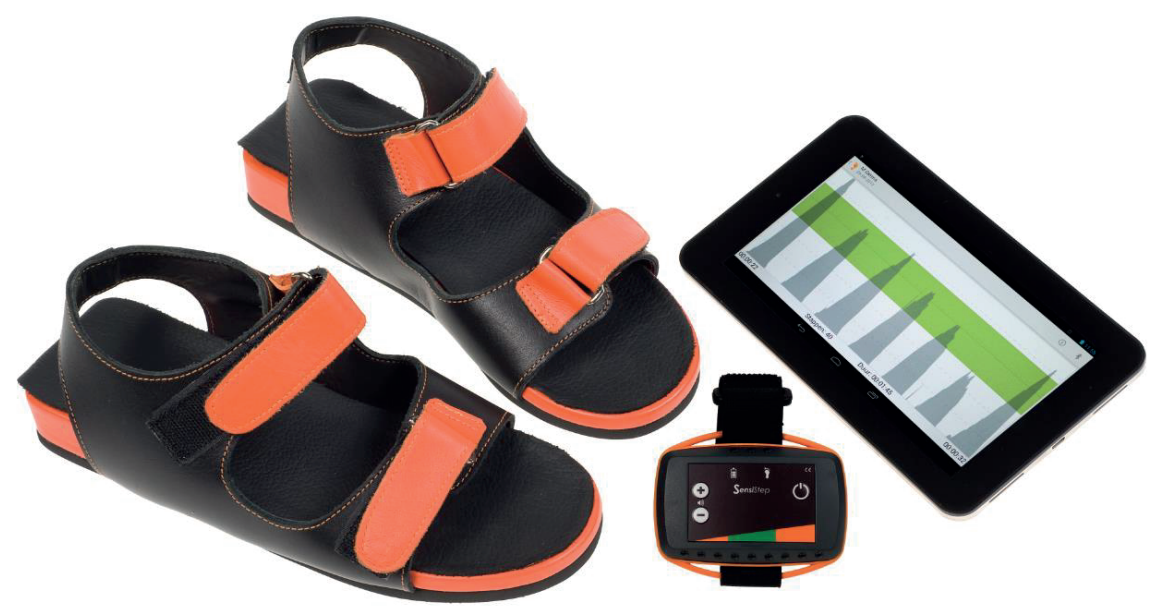

Figure 1. The different parts of the SensiStep system. The force sensor was placed inside the sole of the custom-made sandals. Real-time, visual biofeedback was shown to both the participant and the healthcare professional via the tablet as a green bar (i.e. target weight) and grey step curves (actual weight).

\section{Study protocol}

Patients that fulfilled inclusion criteria were asked to participate in this study. After informed consent was obtained, the participants were randomized into two different groups by block randomization and an allocation ratio of 1:1. Group 1 received real-time, visual feedback on weight-bearing via the SensiStep tablet. In group 2, weight-bearing was measured using SensiStep, but neither the participants nor the physical therapist had insight to the data. The participants in both groups were asked to walk 30 metres in a straight line with SensiStep. Measurements were obtained at pre-defined moments: before the operation, daily after surgery during admission in the hospital and 12 weeks post-operatively at the outpatient follow-up. The post-operative measurements were repeated up to a maximum of five days after surgery, depending on the length of stay in the hospital. Primary outcome parameters were (1) average peak load, (2) average loading rate and (3) average cadence. The secondary outcome parameters (1) Visual Analogue Scale (VAS) pain score (cm), (2) walking distance, (3) duration of usage of walking aids and (4) pain medication, were assessed via questionnaires at 12 weeks follow-up. 


\section{Data processing and statistical analysis}

All raw data were encrypted and stored on a secured Web Portal. The raw data were analysed with MATLAB 2014a. Specific Matlab routines were developed to convert the raw data into the parameters of interest. Data was corrected for the position of the sensor [21]. The average peak load is shown in percentage bodyweight $(\% \mathrm{BW})$, the average loading rate in kilogram per second $(\mathrm{kg} / \mathrm{s})$ and the average cadence in number of steps per second (steps/s). Statistical significance of the SensiStep parameters was determined using multiple $\mathrm{T}$ tests with the Holm-Sidak method. A Chi-square test was used to determine statistical difference of the mobility scores. All data are shown as means with ranges and significance was set at $\alpha=0.05$.

\section{Results}

Between May 2015 and May 2016, a total of 96 patients underwent THA. Of these, 81 underwent the procedure for treatment of primary coxarthrosis. A total of 57 patients were excluded due to either age (21), comorbidities (24), lack of informed consent (8), adverse events (2) or technical failure of the biofeedback system (2). A total of 24 participants were randomized into two groups of twelve participants. Group $1(6 \hat{0}, 69)$ had a mean age of 70.5 years (range: 61-83 years), average weight of $78.3 \mathrm{~kg}$ (range: $45-117$ ) and received real-time, visual feedback from SensiStep. Group $2(6 \hat{\jmath}, 6$ 이) had a mean age of 72.8 years (range: 61-84 years), average weight of $84.3 \mathrm{~kg}$ (range: 70-120) and did not received feedback from SensiStep.

The length of hospital stay after surgery was respectively 3.1 (2-5) and 3.7 (2-5) days for group 1 and $2(\mathrm{P}=0.144)$. Pre-operatively, the participants showed an average peak load of 64.0 (38.4-99.0) \%BW in group 1 and $55.0(21.7-89.1) \% \mathrm{BW}$ in group $2(\mathrm{P}=0.241)$. In all early postoperative days (days 1-4), significantly higher average peak loads were found in group 1, as shown in Figure 2 and Table 1. No significant differences were found in loading rate or cadence for both groups in the early postoperative phase (Figures 3-4 and Table 1). Compared to the pre-operative peak load, group 1 showed a significant improvement at twelve weeks follow up: respectively 64.0 (38.4-99.0) \%BW vs 77.1 (57.3-104.0) \%BW $(\mathrm{P}=0.047)$. In group 2 an increase in peak load from pre-operative to 12 weeks was also seen, but this difference was not significant: respectively 55.0 (21.7-89.1) \%BW vs 68.4 (48.2-91.9) \% $\mathrm{BW}(\mathrm{P}=0.076)$. The loading rate was significantly improved in both groups at twelve weeks follow up compared to the pre-operative situation (group 1: $\mathrm{P}=0.035$ and 
group 2: $\mathrm{P}=0.012$, Figure 3 and Table 1). A significant improvement was also seen in cadence at twelve weeks follow up (group 1: $\mathrm{P}=0.020$ and group 2: $\mathrm{P}=0.031$, Figure 4 and Table 1). Walking distance, as reported by all participants at twelve weeks, was longer in the intervention group. An unrestricted walking distance was reported by half of the participants in the intervention group, whereas only 2 participants reported an unrestricted walking distance in the control group. Also, walking aids were used for a shorter time in the intervention group than in the control group. VAS pain scores and pain medication were similar in both groups (Table 2).

\begin{tabular}{|c|c|c|c|c|c|c|}
\hline \multirow[b]{2}{*}{ Group } & \multicolumn{2}{|c|}{ Peak load (\%BW) } & \multicolumn{2}{|c|}{ Loading rate $(\mathrm{kg} / \mathrm{s})$} & \multicolumn{2}{|c|}{ Cadence (steps/s) } \\
\hline & 1 & 2 & 1 & 2 & 1 & 2 \\
\hline Pre-op & $\begin{array}{l}64.0(38.4- \\
99.0)^{\#}\end{array}$ & $\begin{array}{l}55.0(21.7- \\
89.1)\end{array}$ & $\begin{array}{l}185.6(69.5- \\
330.3)^{\#}\end{array}$ & $\begin{array}{l}158.2(36.1- \\
317.2)^{\#}\end{array}$ & $\begin{array}{l}0.94(0.48- \\
1.25)^{\#}\end{array}$ & $\begin{array}{l}0.89(0.34- \\
1.17)^{\#}\end{array}$ \\
\hline Day 1 & $\begin{array}{l}46.3(20.3- \\
77.6)^{*}\end{array}$ & $\begin{array}{l}31.8(16.3- \\
62.2)^{*}\end{array}$ & $\begin{array}{l}61.7(10.0- \\
109.4)\end{array}$ & $\begin{array}{l}48.0(10.9- \\
112.6)\end{array}$ & $\begin{array}{l}0.39(0.09- \\
0.93)\end{array}$ & $\begin{array}{l}0.30(0.03- \\
0.81)\end{array}$ \\
\hline Day 2 & $\begin{array}{l}52.7(27.4- \\
84.4)^{*}\end{array}$ & $\begin{array}{l}34.5(16.0- \\
56.8)^{*}\end{array}$ & $\begin{array}{l}81.9(23.8- \\
168.2)\end{array}$ & $\begin{array}{l}56.6(15.0- \\
108.3)\end{array}$ & $\begin{array}{l}0.51(0.23- \\
0.89)\end{array}$ & $\begin{array}{l}0.43(0.03- \\
0.91)\end{array}$ \\
\hline Day 3 & $\begin{array}{l}52.8(26.6- \\
73.7)^{*}\end{array}$ & $\begin{array}{l}33.5(17.8- \\
49.8)^{*}\end{array}$ & $\begin{array}{l}99.3(14.9- \\
214.2)\end{array}$ & $\begin{array}{l}50.9(16.7- \\
118.8)\end{array}$ & $\begin{array}{l}0.50(0.18- \\
0.91)\end{array}$ & $\begin{array}{l}0.43(0.04- \\
0.83)\end{array}$ \\
\hline Day 4 & $\begin{array}{l}58.2(35.4- \\
76.7)^{*}\end{array}$ & $\begin{array}{l}35.1(16.7- \\
67.8)^{*}\end{array}$ & $\begin{array}{l}93.1(28.3- \\
177.8)\end{array}$ & $\begin{array}{l}71.615 .9- \\
187.1)\end{array}$ & $\begin{array}{l}0.50(0.24- \\
0.83)\end{array}$ & $\begin{array}{l}0.52(0.05- \\
0.84)\end{array}$ \\
\hline Twelve weeks & $\begin{array}{l}77.1(57.3- \\
104.0)^{\#}\end{array}$ & $\begin{array}{l}68.4(48.2- \\
91.9)\end{array}$ & $\begin{array}{l}266.2(167.1- \\
455.5)^{\#}\end{array}$ & $\begin{array}{l}245.9(116.9- \\
349.5)^{\#}\end{array}$ & $\begin{array}{l}1.15(0.86- \\
1.32)^{\#}\end{array}$ & $\begin{array}{l}1.14(0.75- \\
1.52)^{\#}\end{array}$ \\
\hline
\end{tabular}

Table 1. SensiStep parameters (means with ranges) for both groups. *: significant differences between group 1 and 2 $(\mathrm{P}<0.05)$. ${ }^{*}$ : significant difference between pre-operative and postoperative at twelve weeks $(\mathrm{P}<0.05)$. 


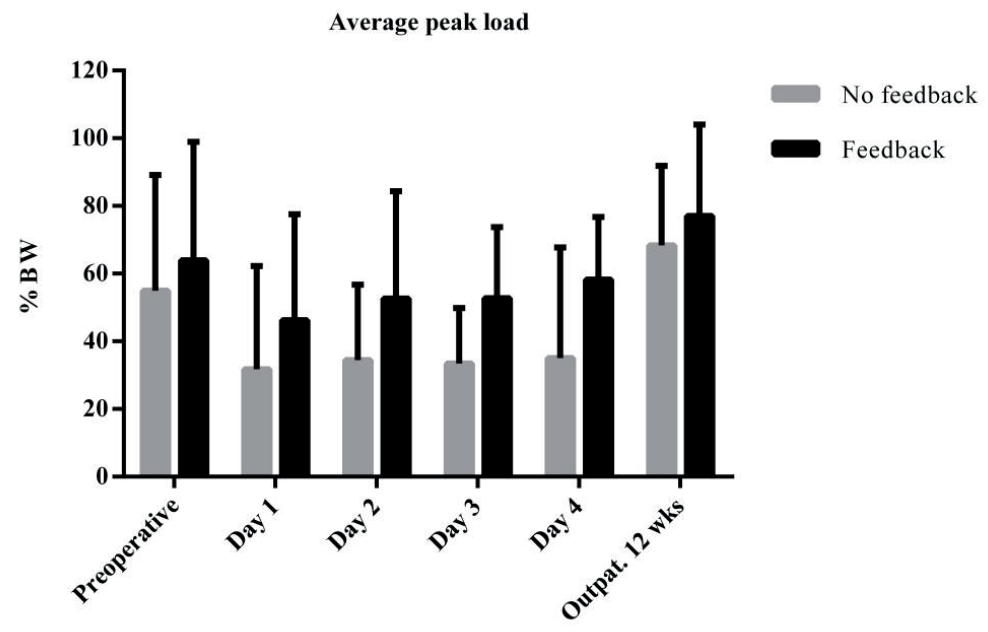

Figure 2. Average peak load measured by SensiStep (means with ranges) for both groups. Significant differences $(\mathrm{P}<0.05)$ between group 1 and 2 were found on day 1,2, 3 and 4 postoperatively. There was a significant increase in average peak load in group 1 between pre-operative and postoperative at twelve weeks $(\mathrm{P}<0.05)$.

\section{Loading Rate}

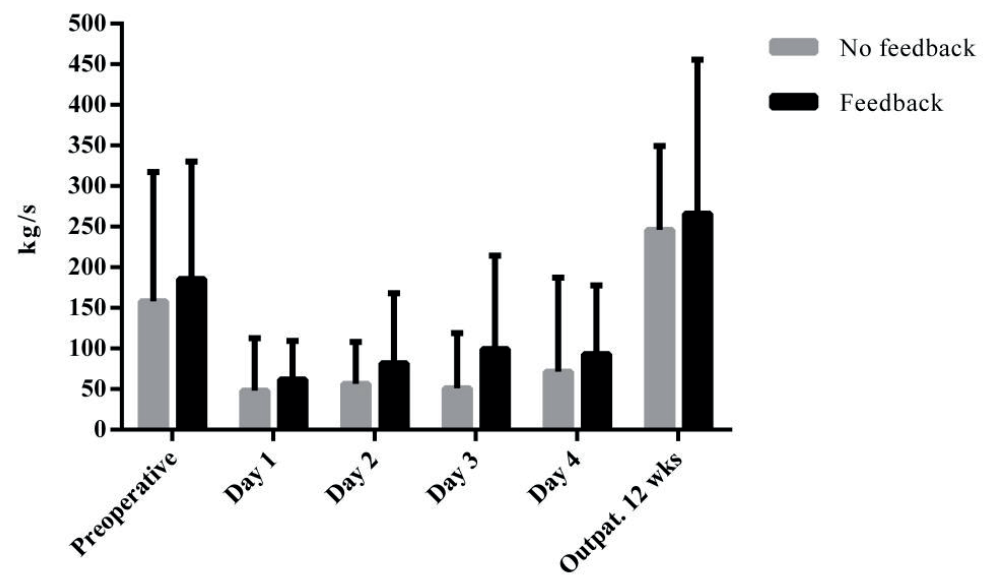

Figure 3. Average loading rate measured by SensiStep (means with ranges) for both groups. There was a significant increase in average loading rate in both groups between pre-operative and postoperative at twelve weeks $(\mathrm{P}<0.05)$. 


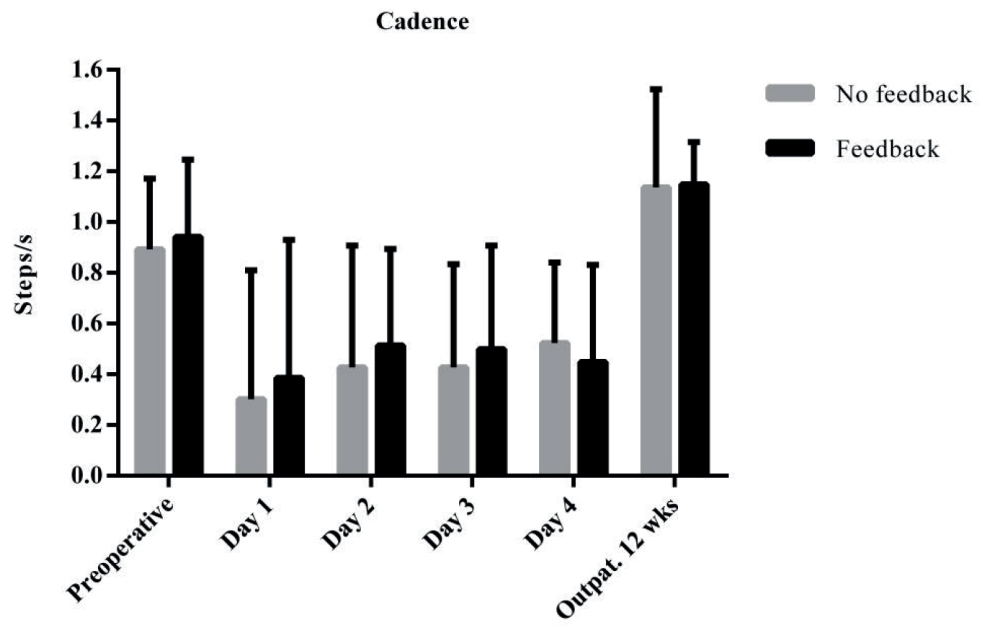

Figure 4. Average cadence measured by SensiStep (means with ranges) for both groups. There was a significant increase in cadence in both groups between pre-operative and postoperative at twelve weeks $(\mathrm{P}<0.05)$.

\begin{tabular}{|l|l|l|l|}
\hline \multicolumn{2}{|l|}{} & Group 1 & Group 2 \\
\hline VAS pain score (cm) & $1.6(0.0-6.0)$ & $0.9(0.0-4.0)$ \\
\hline $\begin{array}{l}\text { Walking distance } \\
\text { (number of patients) }\end{array}$ & Restricted & 5 & 8 \\
\hline $\begin{array}{l}\text { Walking aids } \\
\text { (number of patients) }\end{array}$ & $4-12$ weeks & 3 & 2 \\
\hline $\begin{array}{l}\text { Pain medication } \\
\text { (number of patients) }\end{array}$ & $4-12$ weeks & 2 & 5 \\
\hline
\end{tabular}

Table 2. Mobility at twelve weeks follow up. No significant differences were found in VAS pain score ( $\mathrm{P}=0.419)$, walking distance $(\mathrm{P}=0.160)$, use of walking aids $(\mathrm{P}=0.473)$ or use of pain medication $(\mathrm{P}=0.716)$. However, there tend to be a difference in walking distance, as an unrestricted walking distance was reported by half of the participants in the intervention group, whereas only 2 participants reported an unrestricted walking distance in the control group. Also, walking aids tend to be used for a shorter time in the intervention group than in the control group. 


\section{Discussion}

Total hip arthroplasty is a common and effective procedure in the treatment of coxarthrosis. Postoperative early mobilisation is generally advocated as this prevents complications and has positive effects on coordination and muscle mass [5-7]. However, previous studies have shown limited therapy compliance in weight-bearing in healthy volunteers and patients $[17,18,22]$. In this pilot RCT, we investigated if weight-bearing could be improved by real-time, visual biofeedback in the clinical setting. This RCT has shown a significant improvement in average peak load if real-time visual biofeedback was provided, compared to standard training methods. Although feedback was only given during the first days after surgery, the effect lasted for a longer time, resulting in a significant increase in peak load from pre-operative to 12 weeks in the intervention group.

Literature on rehabilitation after THA is rather limited, specifically if full weight-bearing is allowed. Previous studies focussed mainly on gait symmetry or partial weight-bearing after THA $[15,16,23]$. These studies showed an increased asymmetry in weight-bearing after THA [15,23]. Also, patients were unable to implement partial weight-bearing during mobilisation [24]. Similar to the findings in previous studies, the current study also showed limited therapy compliance if standard training methods were used to train full weightbearing. Therefore, it seems that standard training methods are deficient and need to be improved in future rehabilitation programs. Biofeedback systems are promising as they have shown their potential in rehabilitation.

The potential of using biofeedback systems to improve therapy compliance after THA has also been described, however, again the focus lies on improving gait symmetry or partial weight-bearing $[16,18,22]$. The question remains if the positive effect of biofeedback also applies for patients with unrestricted weight-bearing after THA. In line with previous studies, the current study has shown a positive effect of providing real-time, visual biofeedback on weight-bearing in the clinical setting. Moreover, this is the first study to show that there seemed to be a lasting effect of correct early weight-bearing, as a significant improvement in peak load was found at twelve weeks follow up.

Although biofeedback systems significantly improve therapy compliance in the rehabilitation after THA, it should be noted that none of the participants achieved the target load of $100 \%$ BW. The previously described gait asymmetry after THA could, at least in part, explain this result. Although it is unknown if gait asymmetry is caused by 
postoperative pain, or a residual antalgic gait adopted when patients had severe, painful arthritis in the joint [23], it is a fact that patients avoid weight-bearing on the affected hip $[15,25]$. Therefore, in the ongoing development of biofeedback devices, feedback should be provided on a combination of weight-bearing and gait symmetry to further improve rehabilitation after THA. Furthermore, age and comorbidities should also be considered as reasons for lower peak loads in the early postoperative phase. Elderly patients often use walking aids for stability or other comorbidities, and these could have a major influence on the measured load on the affected limb.

Despite the obvious results of visual biofeedback on peak load, no significant differences were found in the other gait parameters, loading rate and cadence, after biofeedback. The method of feedback may be the reason for these findings. The feedback was provided solely on peak load, instead of loading rate or cadence. Consequently, participants were focused on improving peak load, resulting in little or no positive effect on loading rate and cadence. In addition, previous studies were not consistent regarding the effect of biofeedback systems on loading rate. No effect was seen in the early postoperative phase, while the parameter changed over time if the entire rehabilitation period was investigated [26]. This was attributed to the property that the loading rate is an 'unconscious' gait parameter and this parameter improves when patients recover. This is supported by the results of current study, as the loading rate was significantly improved in both groups at twelve weeks follow up.

Similar findings were found for the cadence. In order to improve loading rate and cadence in the early postoperative phase, targeted feedback about these gait parameters should be provided. However, stimulation of the cadence will result in increase of speed, which in turn may lead to potential harmful situations in elderly people. For this reason, feedback on steps per second was not employed in the current study. In future rehabilitation programs, solutions should be developed to safely provide feedback about the other gait parameters.

Besides the method of feedback, a second limitation is the small number of 24 participants in this RCT. As this was the first study using real-time, visual biofeedback to improve weight-bearing after THA, the effect was unknown prior to the study, and no power calculation was performed. Although we have found significant differences in weightbearing with biofeedback in the early-postoperative phase, it is not possible to draw rigid conclusions about the effect of real-time feedback on healing outcome. For example, 
differences were found at twelve weeks follow up (Table 2), but these were not significant. Possibly a type II error occurred due to the low numbers of participants. Moreover, no validated function tests were used to assess mobility at twelve weeks follow-up, as mobility scores such as the Harris Hip Score possesses ceiling effects and potential clinician bias [8]. Instead, mobility was subjectively scored by the use of questionnaires.

One of the strengths is the prospective, RCT study design, which limits possible confounders in the results. Secondly, weight-bearing was investigated in the clinical setting using an ambulatory biofeedback system. The participants were able to directly adapt weight-bearing to the optimal level during training with the physical therapist in the normal rehabilitation setting. This ensures that results found in this study represent the clinical situation and can directly be translated to the population of interest.

\section{Conclusion}

This pilot RCT has shown a low therapy compliance in the rehabilitation after total hip arthroplasty in elderly. Real-time, visual biofeedback significantly improved weightbearing in the early postoperative phase. This effect remained at twelve weeks follow up. 


\section{References}

[1] R.M. Queen, J.F. Schaeffer, R.J. Butler, C.C. Berasi, S.S. Kelley, D.E. Attarian, M.P. Bolognesi, Does surgical approach during total hip arthroplasty alter gait recovery during the first year following surgery?, J. Arthroplasty. 28 (2013) 1639-1643. doi:10.1016/j.arth.2013.02.008.

[2] N.K. Arden, A. Kiran, A. Judge, L.C. Biant, M.K. Javaid, D.W. Murray, A.J. Carr, C. Cooper, R.E. Field, What is a good patient reported outcome after total hip replacement?, Osteoarthr. Cartil. 19 (2011) 155-162. doi:10.1016/j.joca.2010.10.004.

[3] A. Patel, G. Pavlou, R.E. Mújica-Mota, A.D. Toms, The epidemiology of revision total knee and hip arthroplasty in England and Wales: A comparative analysis with projections for the United States. a study using the national joint registry dataset, Bone Jt. J. 97-B (2015) 1076-1081. doi:10.1302/0301-620X.97B8.35170.

[4] M.W. Zawadsky, M.C. Paulus, P.J. Murray, M.A. Johansen, Early outcome comparison between the direct anterior approach and the mini-incision posterior approach for primary total hip arthroplasty: 150 consecutive cases, J. Arthroplasty. 29 (2014) 1256-1260. doi:10.1016/j.arth.2013.11.013.

[5] A.M. Hol, S. Van Grinsven, C. Lucas, J.L.C. Van Susante, C.J.M. Van Loon, Partial versus unrestricted weight bearing after an uncemented femoral stem in total hip arthroplasty: Recommendation of a concise rehabilitation protocol from a systematic review of the literature, Arch. Orthop. Trauma Surg. 130 (2010) 547-555. doi:10.1007/s00402-0091017-3.

[6] A.G. Robling, A.B. Castillo, C.H. Turner, Biomechanical and Molecular Regulation of Bone Remodeling, Annu. Rev. Biomed. Eng. 8 (2006) 455-98. doi:10.1146/annurev.bioeng.8.061505.095721.

[7] P. Tian, Z. Li, G.-J. Xu, X. Sun, X. Ma, Partial versus early full weight bearing after uncemented total hip arthroplasty: a meta-analysis, J. Orthop. Surg. Res. 12 (2017) 31. doi:10.1186/s13018-017-0527-x.

[8] T.M. Lovelock, N.S. Broughton, C.M. Williams, The Popularity of Outcome Measures for Hip and Knee Arthroplasties, J. Arthroplasty. (2017) 1-4. doi:10.1016/j.arth.2017.08.024.

[9] F.K. Ruiz, M.C. Fu, D.D. Bohl, J.W. Hustedt, M.R. Baumgaertner, M.P. Leslie, J.N. Grauer, Patient Compliance With Postoperative Lower Extremity Touch-down Weight-bearing Orders at a Level I Academic Trauma Center, Orthopedics. 37 (2014) e552-e556. doi:10.3928/01477447-20140528-55.

[10] A. Vasarhelyi, T. Baumert, C. Fritsch, W. Hopfenmüller, G. Gradl, T. Mittlmeier, Partial weight bearing after surgery for fractures of the lower extremity - is it achievable?, Gait Posture. 23 (2006) 99-105. doi:10.1016/j.gaitpost.2004.12.005.

[11] J.W. Hustedt, D.J. Blizzard, M.R. Baumgaertner, M.P. Leslie, J.N. Grauer, Is it possible to train patients to limit weight bearing on a lower extremity?, Orthopedics. 35 (2012) e31-7. doi:10.3928/01477447-20111122-14.

[12] B.J. Braun, N.T. Veith, M. Rollmann, M. Orth, T. Fritz, S.C. Herath, J.H. Holstein, T. Pohlemann, Weightbearing recommendations after operative fracture treatment-fact or fiction? Gait results with and feasibility of a dynamic, continuous pedobarography insole, Int. Orthop. (2017) 1-6. doi:10.1007/s00264-017-3481-7.

[13] H.L.P. Hurkmans, J.B.J. Bussmann, E. Benda, J. a N. Verhaar, H.J. Stam, Techniques for measuring weight bearing during standing and walking, Clin. Biomech. 18 (2003) 576-589. doi:10.1016/S0268-0033(03)00116-5. 
[14] S.C. White, R.M. Lifeso, Altering asymmetric limb loading after hip arthroplasty using real-time dynamic feedback when walking, Arch. Phys. Med. Rehabil. 86 (2005) 1958-1963. doi:10.1016/j.apmr.2005.04.010.

[15] B. Cichy, M. Wilk, Z. Sliwiński, Changes in gait parameters in total hip arthroplasty patients before and after surgery., Med. Sci. Monit. 14 (2008) CR159-R169.

[16] Z. Pataky, D. De León Rodriguez, A. Golay, M. Assal, J.P. Assal, C.A. Hauert, Biofeedback Training for Partial Weight Bearing in Patients After Total Hip Arthroplasty, Arch. Phys. Med. Rehabil. 90 (2009) 1435-1438. doi:10.1016/j.apmr.2009.02.011.

[17] M.C. Fu, L. DeLuke, R. a Buerba, R.E. Fan, Y.J. Zheng, M.P. Leslie, M.R. Baumgaertner, J.N. Grauer, Haptic biofeedback for improving compliance with lower-extremity partial weight bearing., Orthopedics. 37 (2014) e993-8 doi:10.3928/01477447-20141023-56.

[18] E. Hershko, C. Tauber, E. Carmeli, Biofeedback Versus Physiotherapy in Patients With Partial Weight-Bearing, Am. J. Orthop. 37 (2008) 92-96.

[19] M. Raaben, H.R. Holtslag, L.P.H. Leenen, R. Augustine, T.J. Blokhuis, Real-time visual biofeedback during weight bearing improves therapy compliance in patients following lower extremity fractures, Gait Posture. 59 (2018) 206210. doi:10.1016/j.gaitpost.2017.10.022.

[20] M. Raaben, H. Holtslag, R. Augustine, R. van Merkerk, B. Koopman, T. Blokhuis, Technical Aspects and Validation of a New Biofeedback System for Measuring Lower Limb Loading in the Dynamic Situation, Sensors. 17 (2017) 658. doi:10.3390/s17030658.

[21] B.L. Davis, P.R. Cavanagh, Decomposition of superimposed ground reaction forces into left and right force profiles, J. Biomech. 26 (1993) 593-597. doi:10.1016/0021-9290(93)90020-F.

[22] H.L. Hurkmans, J.B. Bussmann, E. Benda, J.A. Verhaar, H.J. Stam, Effectiveness of audio feedback for partial weight-bearing in and outside the hospital: A randomized controlled trial, Arch. Phys. Med. Rehabil. 93 (2012) 565-570. doi:10.1016/j.apmr.2011.11.019.

[23] J.L. McCrory, S.C. White, R.M. Lifeso, Vertical ground reaction forces: objective measures of gait following hip arthroplasty, Gait Posture. 14 (2001) 104-109. doi:10.1016/S0966-6362(01)00140-0.

[24] M. Tveit, J. Kärrholm, Low effectiveness of prescribed partial weight bearing. Continuous recording of vertical loads using a new pressure-sensitive insole., J. Rehabil. Med. 33 (2001) 42-46.

[25] A.M. Ewen, S. Stewart, A. St Clair Gibson, S.N. Kashyap, N. Caplan, Post-operative gait analysis in total hip replacement patients-A review of current literature and meta-analysis, Gait Posture. 36 (2012) 1-6. doi:10.1016/j.gaitpost.2011.12.024.

[26] A. Bakker, T.J. Blokhuis, M.D.M.E. Meeks, H.J. Hermens, H.R. Holtslag, Dynamic weight loading in older people with hip fracture., J. Rehabil. Med. (2014) 1-4. doi:10.2340/16501977-1793. 


\section{PART}
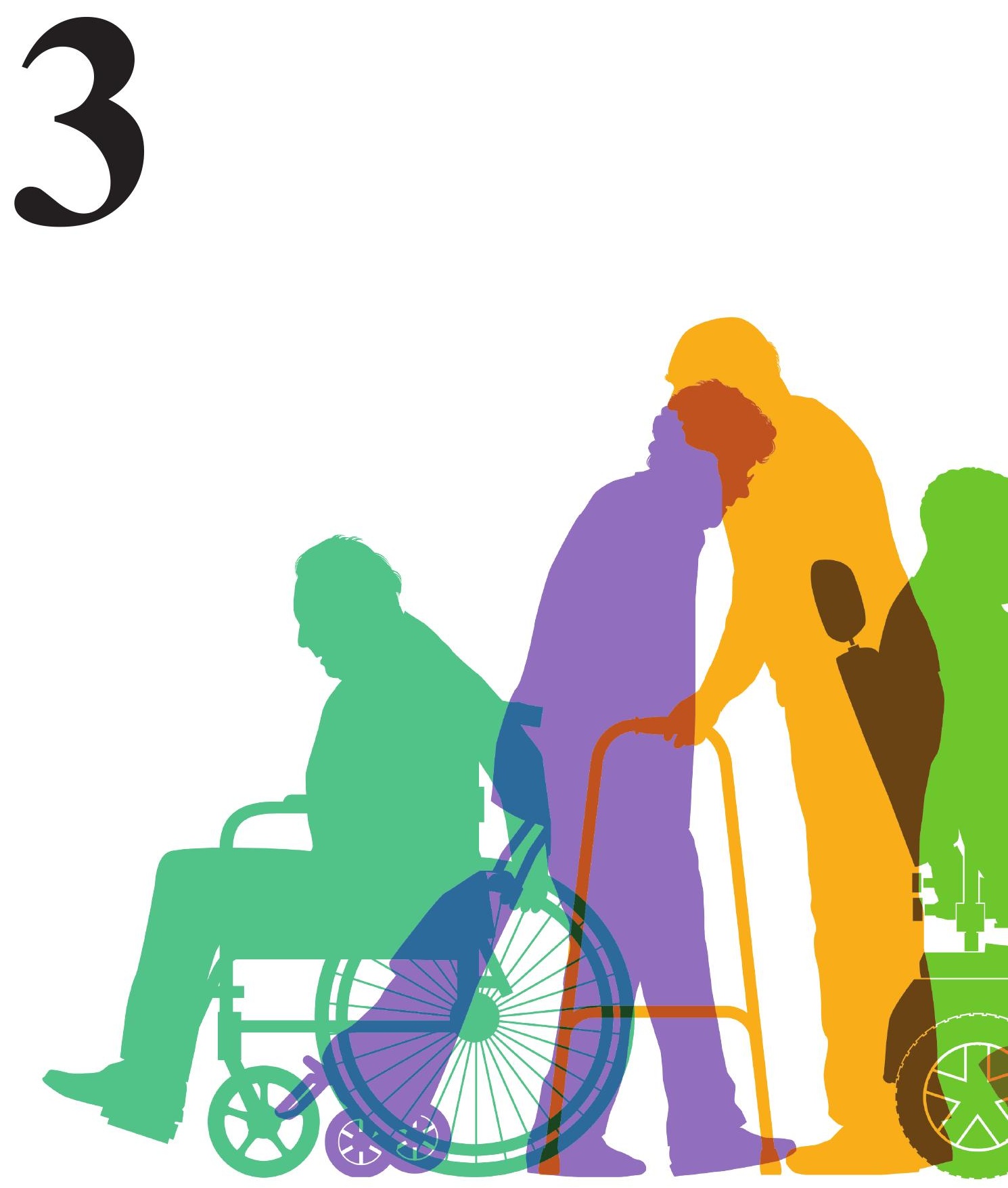


\section{Biofeedback in the elderly population}

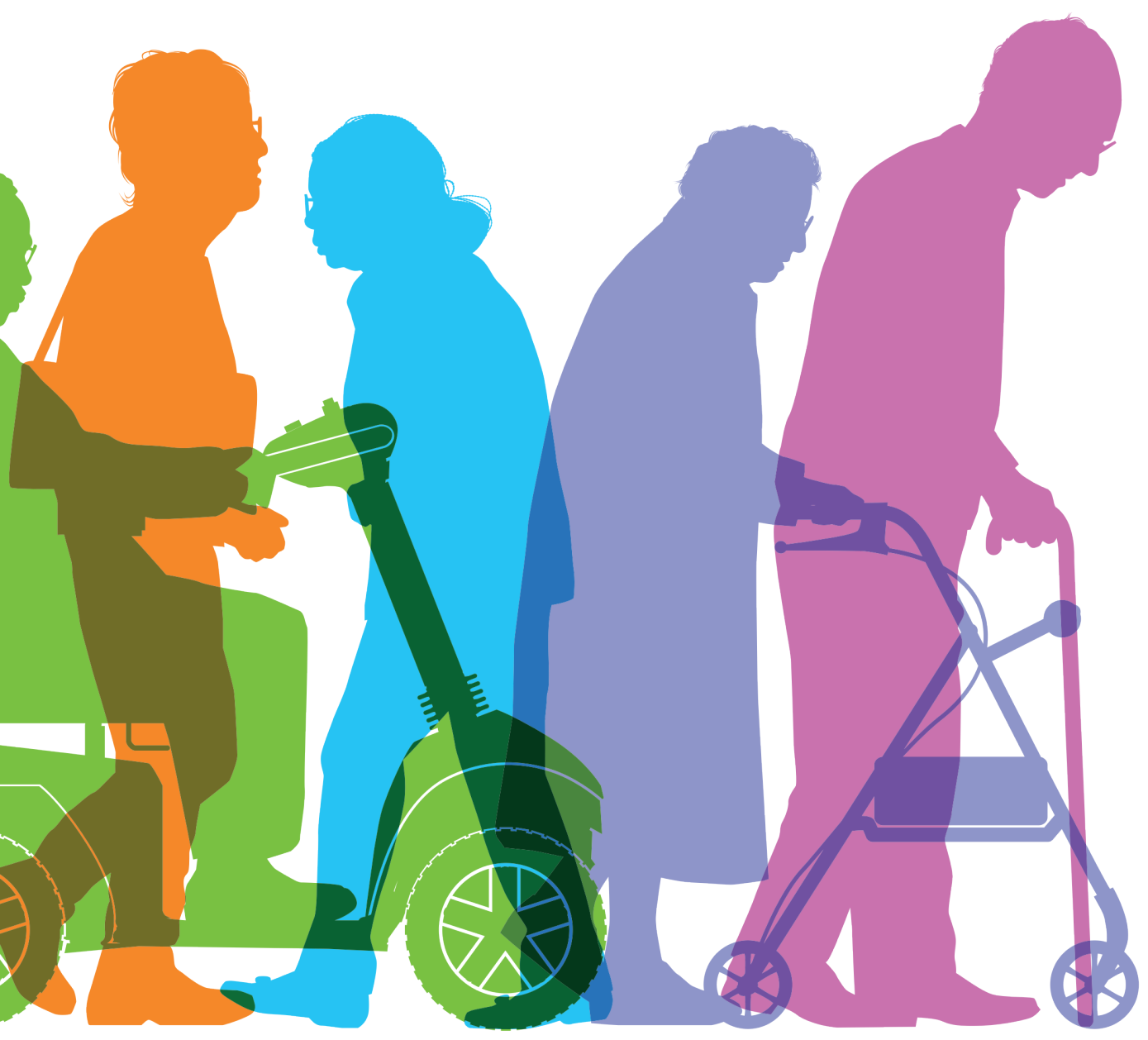


CHAPTER
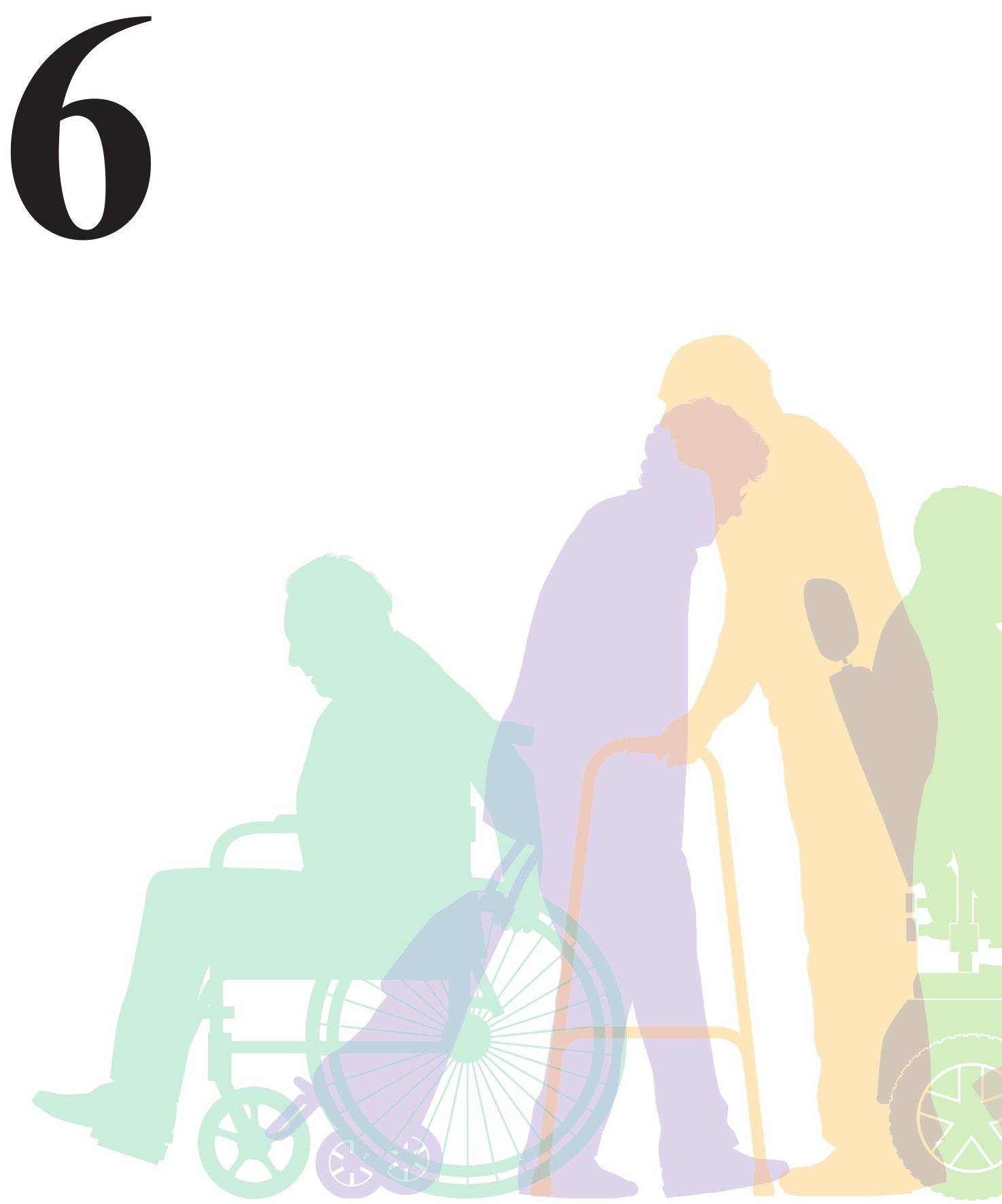
Innovative Measurement Of Rehabilitation Progress In Elderly With A Hip Fracture: A New Endpoint

2018 IEEE Conference on Antenna Measurements \& Applications (CAMA), Vasteras, 2018, pp 1-4

M. Raaben, S. Redzwan, R. Augustine, T.J. Blokhuis 


\begin{abstract}
Background: The worldwide ageing population leads to an increase in the incidence of hip fractures in elderly. Weight bearing is important to improve outcome, but rehabilitation is often hampered by the absence of proper rehabilitation tools. The aim of current study is to gain insight in important gait parameters and the rehabilitation progress of elderly with a hip fracture using an innovative biofeedback system (SensiStep).

Materials and Methods: Force measurements were performed in 113 participants during a 30-meter walk with SensiStep. Also, two mobility tests and pain score were assessed in these participants. In 10 participants the measurements were repeated after one year follow up, and 40 healthy volunteers were measured.

Results: The parameters peak force and step duration improved towards the end of rehabilitation. A new and objective endpoint of rehabilitation emerges from these two parameters. Moreover, a differentiated approach in rehabilitation could be developed based on the first training session.
\end{abstract}

Conclusions: This could lead to individual rehabilitation programs and thereby improved outcome after hip fractures in elderly.

Keywords: hip fracture, weight bearing, rehabilitation, biofeedback, SensiStep. 


\section{Introduction}

The life expectancy of the world population is increasing every year. This also leads to an increasing survival of frail people with low bone quality and high risk of falls, leading to an exponentially increasing incidence of hip fractures in older people [1].

Due to the high morbidity and mortality, hip fractures are a major health concern and have significant impact on society [2]. Proper rehabilitation programmes after hip fractures could improve outcome, thereby increasing the number of patients that return to society and reducing costs.

Previous studies have shown that early weight bearing is important in rehabilitation after lower extremity fractures, including hip fractures [3]. Especially full weight bearing is generally advocated as this leads to an increase in coordination, bone mass and muscle strength $[4,5]$. It is known, however, that therapy compliance is limited due to the absence of proper rehabilitation tools [6,7]. Recently, biofeedback systems have shown their clinical potential, as significant improvements in weight bearing were found $[8,9]$.

These biofeedback systems are promising for rehabilitation after hip fractures in elderly as well. However, before these systems can be used we first need to know the normal gait patterns and analyse which parameters are important to guide the biofeedback. Therefore, the aim of current study is to gain insight in the normal gait patterns and rehabilitation progress after hip fractures in elderly. In addition, it will be evaluated how these gait patterns are changed at one year follow up and how they are related to healthy volunteers.

\section{Materials and Methods}

\section{SensiStep}

The innovative biofeedback system SensiStep (Evalan BV, Amsterdam, The Netherlands) is used to gain insight in normal gait patterns after hip fractures in elderly. SensiStep was previously validated and able to continuously and reliably monitor gait in the ambulant setting [10]. In short, SensiStep uses a wireless, insole force sensor based on the Hall-effect to measure the exerted force on the lower extremity during gait. In addition, biofeedback can be provided in real-time to both the patient and the healthcare provider via a wrist device (i.e. Sensi) and a tablet. 


\section{Inclusion and exclusion criteria}

SensiStep was used to analyse the normal gait patterns in elderly patients in geriatric rehabilitation centers in the Netherlands and Sweden. In order to create a homogenic group, the following inclusion criteria were used:

- $\quad$ Age $\geq 60$ years.

- Hip fracture following low energy trauma (i.e. fall from standing position).

- Full weight bearing allowed after (surgical) treatment of the fracture.

- Expected rehabilitation of $\geq 2$ weeks in the geriatric rehabilitation center.

- Bodyweight $\leq 120 \mathrm{~kg}$.

Patients that met the following criteria were excluded from the study:

- Cognitive impairment, defined as a score $<18$ on the Mini Mental State Examination (MMSE).

- Readmission to the hospital within 2 weeks after inclusion in the study, for example due to infectious complications.

- Co-morbidities that affected gait significantly prior to the hip fracture.

\section{Study protocol}

Patients that met the inclusion criteria were asked to participate in the study. After informed consent was signed, the MMSE was obtained. The MMSE is an objective score to assess the cognitive state of the participants, and a score $<18$ indicates severe cognitive impairment [11].

All participants followed the institutional protocol for physical therapy after hip fractures in elderly. Study data were gathered by a standardized study protocol. The normal gait patterns were obtained by daily force measurements using the SensiStep in a 30-meter walk. Neither the participants nor the supervising healthcare professional did receive realtime biofeedback from SensiStep to prevent bias by (unconscious) adjustments of the normal gait patterns. Finally, two mobility tests were performed, i.e. Functional Ambulation Categories (FAC, 1x per week) and de Morton Mobility Index (DEMMI, 1x per 2 weeks). The pain score was determined by the Visual Analogue Scale (VAS, 1x per week). 
One year follow up and healthy volunteers

Study participants $(n=10)$ of two centers were asked to perform a 30-meter walk with SensiStep at one year follow up to gain insight in the developments of gait pattern after discharge from the rehabilitation center. In addition, the normal gait patterns of healthy volunteers (young and $\geq 60$ years, $n=40$ ) were obtained during a 30 -meter walk with SensiStep according to the study protocol.

\section{Data analysis}

All gathered data were encrypted and stored on the SensiStep Web Portal. All data were anonymized and made available to the investigators for research purposes. The raw data were then converted into parameters of interest by specific developed Matlab routines (Matlab R2014a, MathWorks) based on previous studies [12]. All data are shown as mean $\pm \mathrm{SD}$.

\section{Results}

113 participants were included between February 2016 and June 2017. Demographics are shown in Table 1. The average MMSE score of the participants was 26.7 \pm 3.3 . Type of surgery were (total) hip prosthesis $(n=48)$, gamma nail $(n=24)$, dynamic hip screw $(n=8)$, pertrochanteric femur nail $(n=22)$, cannulated screws $(n=3)$ and other $(n=8)$. Most of the participants had multiple comorbidities.

The normal gait patterns were analysed by the previously defined parameters of interest: (1) peak force; (2) step duration; (3) heel stance time; (4) impulse; (5) loading rate. The parameters (3) heel stance time; (4) impulse and (5) loading rate did not show correlations with the rehabilitation progress (data not shown). In contrast, the parameters (1) peak force and (2) step duration showed improvements towards the end of rehabilitation. All participants had higher peak forces and faster steps one day before discharge from the geriatric rehabilitation center (Figure 1). Moreover, where the peak force and step duration displayed substantial variability in the first week, most steps on the day of discharge were found to be in a single point (Figure 2). The convergence of all these steps indicates an objective endpoint of rehabilitation after hip fractures in elderly. 


\begin{tabular}{|l|l|}
\hline \multicolumn{2}{|c|}{ Participant demographics } \\
\hline Age & $82.0 \pm 8.8$ \\
\hline Gender (male : female) & $26: 87$ \\
\hline Weight & $68.9 \pm 14.5$ \\
\hline BMI & $23.0 \pm 1.0$ \\
\hline
\end{tabular}

Table 1. Participant demographics $(\mathrm{n}=113)$.

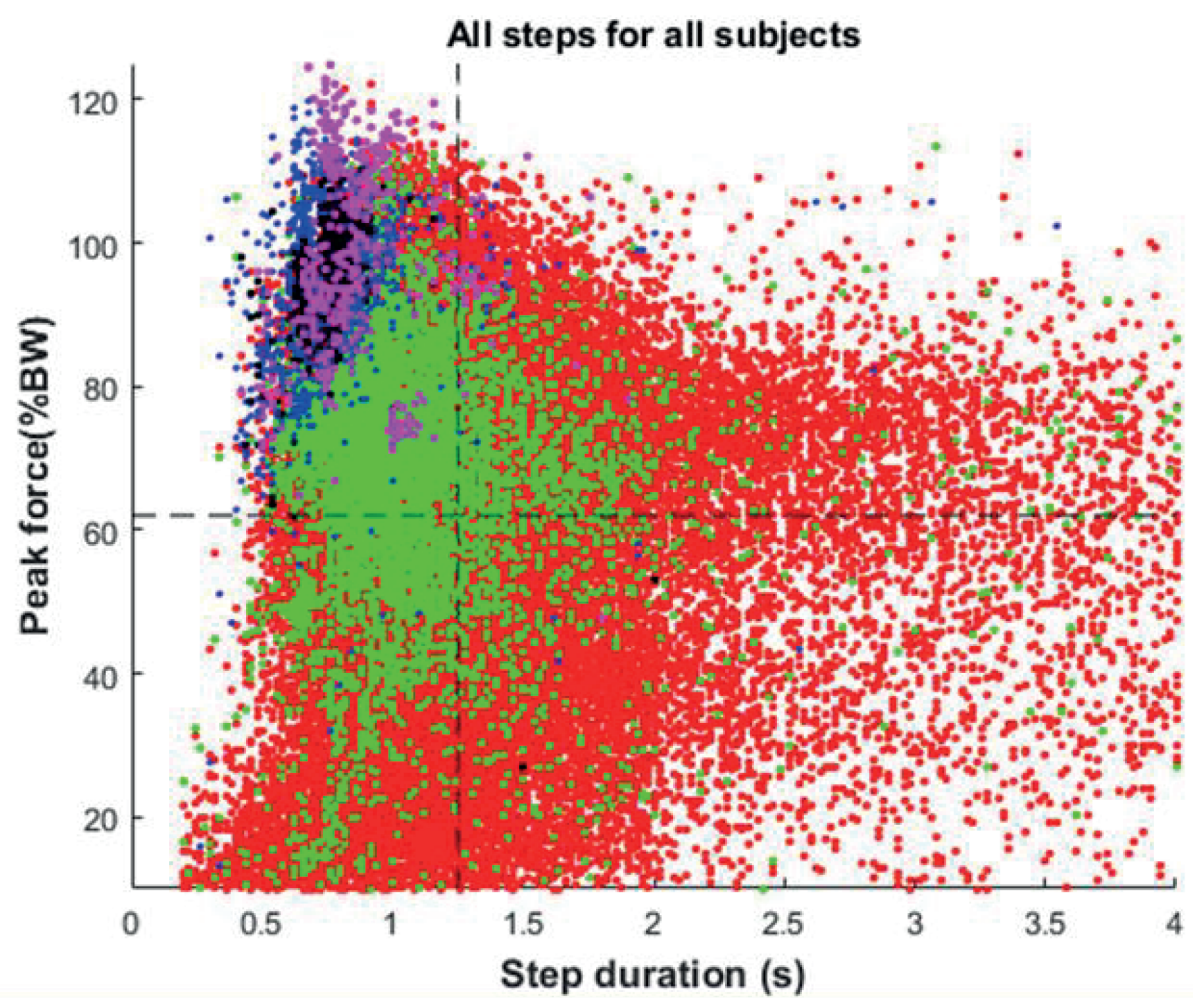

Figure 1. All steps for all participants, healthy volunteers and one year follow up. The red dots represent all steps from all study participants from all sessions. The green dots represent all steps from all study participants one day before discharge from the rehabilitation center. Both the step duration (x-axis) as the peak force (y-axis) improve from the start of rehabilitation (red) to the end of rehabilitation (green). Still, the healthy volunteers (blue and black) were found to have better step duration and peak force compared to study participants at discharge (green). No differences were found between $>60$ years and young healthy volunteers (respectively blue and black). Interestingly, improvements were found in both gait parameters at one year follow up (purple) compared to the day of discharge (green). No differences were found between healthy volunteers (blue and black) and one year follow up (purple). The dashed lines represent the median of step duration and peak force based on the first SensiStep session from all study participants. Based on the first SensiStep session, study participants could be categorized into four different groups: (1) high peak force and high step duration; (2) high peak force, low step duration; (3) low peak force, high step duration; (4) low peak force, low step duration. 


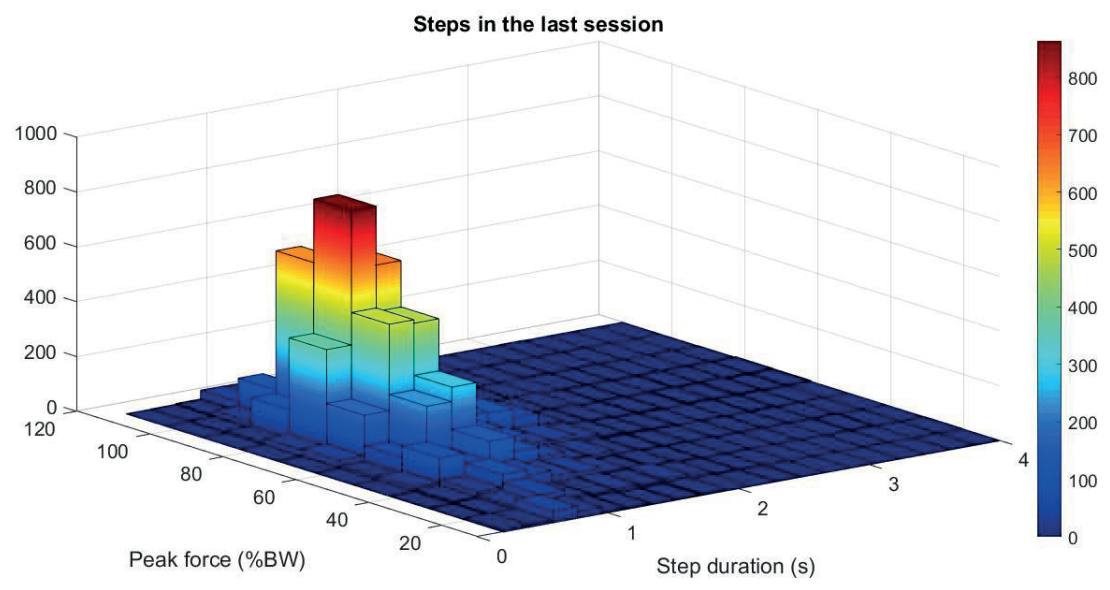

Figure 2. A new endpoint of rehabilitation can be defined by the parameters peak force and step duration. Most steps are concentrated around a single point at the day of discharge, indicating the end of rehabilitation. As these gait parameters can objectively be measured by SensiStep, it could replace the current endpoint of rehabilitation, which is partly experience based and partly based on subjective mobility tests.

8 young (31.7y \pm 7.9$)$ and 32 elderly $(69.6 \mathrm{y} \pm 8.6)$ healthy volunteers were measured with SensiStep and the peak force and step duration were compared to the study participants. As shown in figure 1, the healthy volunteers were found to exert higher peak forces and faster steps than participants measured one day before discharge. No differences were found between young and elderly healthy volunteers. Interestingly, the participants at one year follow up showed similar peak forces and step durations as the healthy volunteers (Figure $1)$.

Two validated mobility tests were performed to gain additional insight in the rehabilitation progress. The FAC score improved over time from 3 at the start to 4 at the end of the rehabilitation. Similar results were found for the DEMMI score: 41 to 57 respectively. The amount of pain experienced by the participant decreased over time based on VAS score from 5 to 3 .

\section{Discussion}

Hip fractures have a high morbidity and mortality and have thereby a significant impact on society. From previous studies it is known that (early) weight bearing is important to improve outcome. However, insight in weight bearing and other gait patterns is limited due to the absence of proper rehabilitation tools. In the current study SensiStep was used to 
analyse the rehabilitation progress and clear changes were found in the peak force and step duration.

Analysis of all participants showed that the peak force and step duration gradually changed during rehabilitation and converge towards a clear point, where most steps on the day before discharge are located in a single point. We therefore propose this point as the new endpoint of rehabilitation, which can objectively be determined by SensiStep. This could replace the current endpoint of rehabilitation, which is partly experience based and partly based on subjective mobility tests.

Remarkable is that the new endpoint of rehabilitation has lower peak forces and step durations compared to healthy volunteers. This indicates that patients are unable the achieve their pre-fracture mobility, as was also found in previous studies where a high morbidity was found after hip fractures [2]. However, both gait parameters improved at one year follow up and were similar to healthy volunteers. Although the number of patients measured at one year follow up was low, this suggests that patients continue their rehabilitation at home and reach their pre-fracture mobility at one year after their injury.

Previous studies found other predictive gait parameters as well, such as the heel stance time, impulse and loading rate [12]. These findings were not confirmed in the current study. Two reasons may explain these different findings. First, significantly more participants were included in the current study $(n=113)$ compared to the previous study $(n=21)$. Second, the study protocol varies, as current study evaluates gait in a 30-meter walk while previous studies evaluated gait based on significantly longer walks.

The international, multicenter design of current study ensures that the results can be translated to other geriatric rehabilitation centers worldwide. Also, the strict inclusion criteria ensure homogeneity of the study population. Although it was hypothesized that homogeneity would result in similar gait patterns, the participants showed differences in gait parameters based on the first SensiStep session, as shown by the red steps in Fig 1. This means that the starting point for rehabilitation is very variable, despite the homogeneity of the population. The participants can therefore be divided into four different groups based on the first training session: (1) high peak force and high step duration; (2) high peak force, low step duration; (3) low peak force, high step duration; (4) low peak force, low step duration. This finding offers the opportunity to develop a differentiated approach in rehabilitation, instead of a one size fits all-approach. For example, patients with 
a good start (i.e. group 1) could follow a less intensive training regime than patients with a bad start (i.e. group 4). In addition, patients in group 2 could focus on improving the step duration, while patients in group 3 could focus on improving the peak force. Such an individual rehabilitation program could lead to effective rehabilitation and improved outcome after hip fractures in elderly.

\section{Conclusion}

The peak force and step duration are important parameters in the rehabilitation after hip fractures in elderly. A new and objective endpoint of rehabilitation can be defined based on these two parameters. Moreover, a differentiated approach in rehabilitation can be determined in the first training session to improve healing outcome. These findings show that biofeedback systems are promising in future rehabilitation programs regarding, but not limited to, hip fractures in elderly. 


\section{References}

[1] K. Rapp, G. Büchele, K. Dreinhöfer, B. Bücking, C. Becker, and P. Benzinger, "Epidemiology of hip fractures: Systematic literature review of German data and an overview of the international literature," Z. Gerontol. Geriatr., no. November 2017, 2018.

[2] C. M. M. Peeters, E. Visser, C. L. P. Van De Ree, T. Gosens, B. L. Den Oudsten, and J. De Vries, "Quality of life after hip fracture in the elderly: A systematic literature review,” Injury, vol. 47, no. 7, pp. 1369-1382, 2016.

[3] I. B. Houben, M. Raaben, M. van Basten Batenburg, and T. J. Blokhuis, "Delay in weight bearing in surgically treated tibial shaft fractures is associated with impaired healing: a cohort analysis of 166 tibial fractures," Eur. J. Orthop. Surg. Traumatol., pp. 1-8, 2018.

[4] J. Klein-Nulend, R. G. Bacabac, and M. G. Mullender, "Mechanobiology of bone tissue,” Pathol. Biol., vol. 53, no. 10 , pp. 576-580, 2005.

[5] A. G. Robling, A. B. Castillo, and C. H. Turner, "Biomechanical and Molecular Regulation of Bone Remodeling,” Annu. Rev. Biomed. Eng., vol. 8, pp. 455-98, 2006.

[6] A. Vasarhelyi, T. Baumert, C. Fritsch, W. Hopfenmüller, G. Gradl, and T. Mittlmeier, "Partial weight bearing after surgery for fractures of the lower extremity - is it achievable?," Gait Posture, vol. 23, no. 1, pp. 99-105, 2006.

[7] F. K. Ruiz, M. C. Fu, D. D. Bohl, J. W. Hustedt, M. R. Baumgaertner, M. P. Leslie, and J. N. Grauer, "Patient Compliance With Postoperative Lower Extremity Touch-down Weight-bearing Orders at a Level I Academic Trauma Center," Orthopedics, vol. 37, no. 6, pp. e552-e556, 2014.

[8] M. Raaben, H. R. Holtslag, L. P. H. Leenen, R. Augustine, and T. J. Blokhuis, "Real-time visual biofeedback during weight bearing improves therapy compliance in patients following lower extremity fractures," Gait Posture, vol. 59, no. October 2017, pp. 206-210, 2018.

[9] M. Raaben, H. C. Vogely, and T. J. Blokhuis, "Real-time visual biofeedback to improve therapy compliance after total hip arthroplasty: A pilot randomized controlled trial,” Gait Posture, vol. 61, no. January, pp. 306-310, 2018.

[10] M. Raaben, H. Holtslag, R. Augustine, R. van Merkerk, B. Koopman, and T. Blokhuis, “Technical Aspects and Validation of a New Biofeedback System for Measuring Lower Limb Loading in the Dynamic Situation,” Sensors, vol. 17, no. 3, p. $658,2017$.

[11] T. N. Tombaugh and N. J. McIntyre, "The mini-mental state examination: a comprehensive review., Journal of the American Geriatrics Society, vol. 40, no. 9. pp. 922-935, 1992.

[12] A. Bakker, T. J. Blokhuis, M. D. M. E. Meeks, H. J. Hermens, and H. R. Holtslag, "Dynamic weight loading in older people with hip fracture.," J. Rehabil. Med., pp. 1-4, 2014. 
CHAPTER
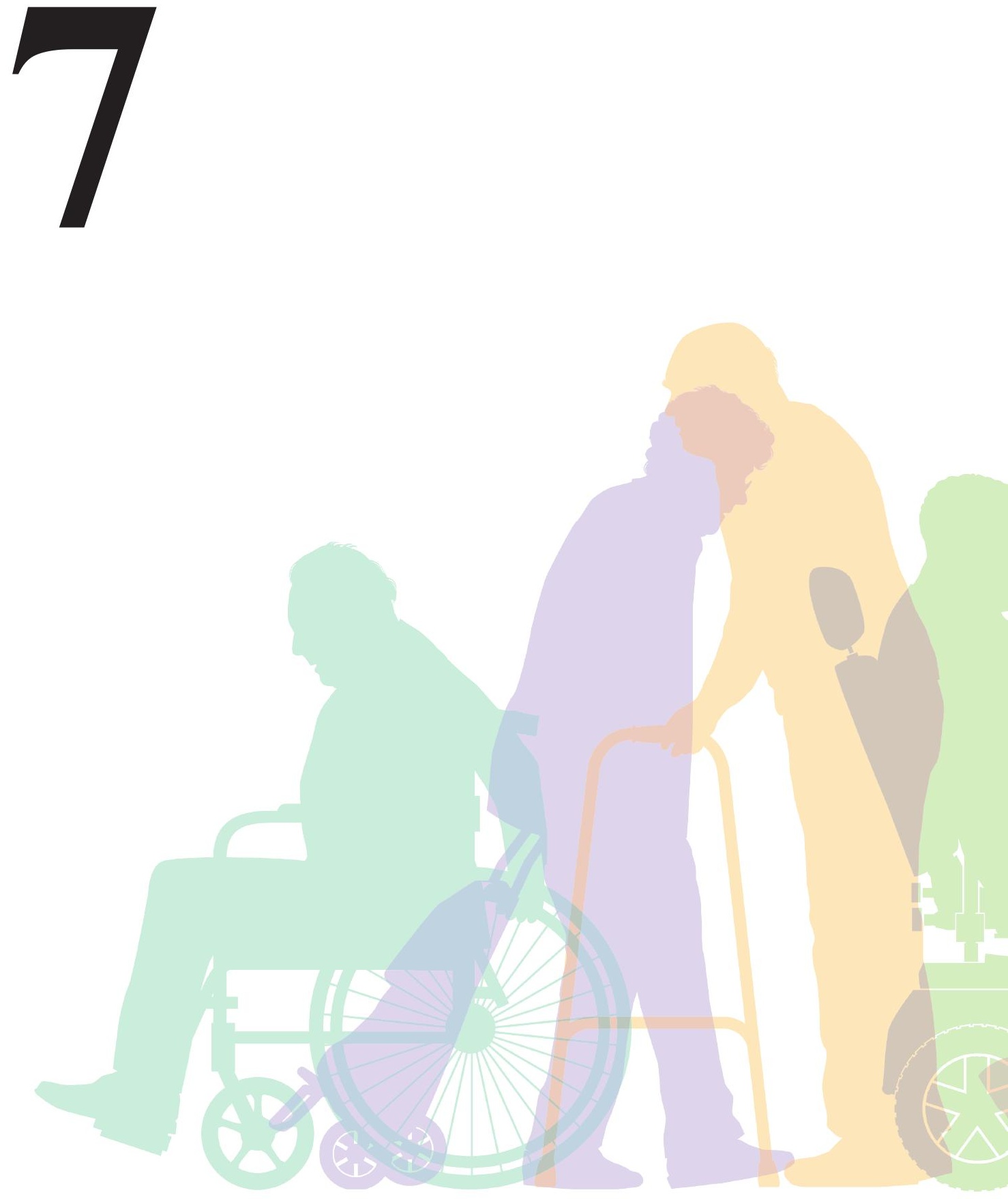
COMplex Fracture Orthopedic Rehabilitation (COMFORT) - Real-time visual biofeedback on weight bearing versus standard training methods in the treatment of proximal femur fractures in the elderly: study protocol for a multicenter randomized controlled trial

Trials, December 2018, Volume 19, Issue 1, pp 220

M. Raaben, S. Redzwan, R. Augustine, T.J. Blokhuis 


\begin{abstract}
Background: Proximal femur fractures are a common injury after low energy trauma in elderly. Most rehabilitation programs are based on restoring mobility and early resumption of weight bearing. However, therapy compliance is low in patients following lower extremity fractures. Moreover, little is known about the relevance of gait parameters and how to steer the rehabilitation after proximal femur fractures in elderly. Therefore, the aim of this prospective, randomized controlled trial is to gain insight in gait parameters and evaluate if real-time, visual biofeedback can improve therapy compliance after proximal femur fractures in elderly.
\end{abstract}

Materials and Methods: This is a two-arm, parallel-design, prospective, randomized controlled trial. Inclusion criteria are age $\geq 60$, a proximal femur fracture following low energy trauma and unrestricted weight bearing. Exclusion criteria are cognitive impairment and limited mobility prior to trauma. Participants are randomized into either the control group, which receives care as usual, or the intervention group, which receives real-time, visual biofeedback about weight bearing during gait in addition to care as usual.

Results: Spatio-temporal gait parameters will be measured in 94 participants per group during a 30 meter walk with an ambulatory biofeedback system (SensiStep). The progress of rehabilitation will be evaluated by the primary outcome parameters maximum peak load and step duration in relation to the discharge date. Secondary outcome parameters include other spatio-temporal gait parameters in relation to discharge date. Furthermore, the gait parameters will be related to three validated clinical tests: Elderly Mobility Scale, Functional Ambulation Categories and Visual Analogue Scale. The primary hypothesis is that participants in the intervention group will show improved and faster rehabilitation compared to the control group.

Conclusions: The first aim of this multicenter trial is to investigate the normal gait patterns after proximal femur fractures in elderly. The use of biofeedback systems during rehabilitation after proximal femur fractures in elderly is promising, and therefore, the second aim is to investigate the effect of real-time, visual biofeedback on gait after proximal femur fractures in elderly. This could lead to improved outcome. In addition, analysis of the population may indicate characteristics of subgroups that benefit from feedback, making a differentiated approach in rehabilitation strategy possible. 
Keywords: proximal femur fracture, weight-bearing, biofeedback, gait analysis, SensiStep, fracture rehabilitation. 


\section{Background}

Due to the ageing population, the worldwide incidence of hip fractures will rise from 1.66 million in 1990 to 6.26 million by 2050 [1]. Hip fractures have a high mortality and high morbidity [2]. Loss of function is common after a hip fracture and patients experience difficulties in their return to society or to their previous habitat. This leads to long-term care in rehabilitation facilities, which is the largest component in the total costs in the treatment of hip fracture patients [3].

Most rehabilitation programs are based on restoration of mobility and early resumption of weight bearing [4]. Weight bearing is important as it helps to maintain muscle and bone mass [5]. In addition, early weight bearing could also lead to improved fracture healing through mechanotransduction [6]. Therefore, early (partial) weight bearing is generally advocated and trained with walking aids under supervision of a physical therapist. Bathroom scales are commonly used to guide the amount of weight bearing to the patient. This method is not only unreliable, but also not very helpful for patients as information about the amount of weight bearing is only provided in the static situation and lost in the dynamic situation.

Although the bathroom scale is still the gold standard in most rehabilitation clinics, the introduction of biofeedback systems is promising as information can be provided in the dynamic situation [7]. Previous studies have already shown improvement in partial and full weight bearing when biofeedback systems were used, compared to standard training methods $[8,9]$. For example, visual biofeedback has shown positive results in the treatment after Parkinsons's disease, in the late period after stroke and after cerebral palsy [10-12]. Also, auditory and visual biofeedback have shown significant improvements in weight bearing after lower extremity fractures $[9,13,14]$. Although these results seem promising, the effect of real-time, visual biofeedback on weight bearing during rehabilitation after proximal femur fractures in elderly is still unknown.

Therefore, in this randomized controlled trial (RCT), real-time visual biofeedback will be provided to the patient and healthcare professional in the clinical setting by the ambulatory biofeedback system SensiStep (Evalan BV, Amsterdam, The Netherlands). This biofeedback system was previously validated in static and dynamic situations [15]. In short, the system consists of an in-sole force sensor, which is able to measure weight bearing reliably and continuously during gait. Generated peak loads are directly translated into a 
LED-signal, to provide real-time feedback to the patient. The same signal is sent to a tablet, resulting in a real-time graphical illustration of each step shown to the healthcare professional. By using this biofeedback system, patients can be guided to the optimal level of weight bearing. This potentially leads to better functional outcome, as well as improved fracture healing through mechanotransduction.

In this RCT it will be investigated whether elderly patients recover faster or better after proximal femur fractures if they receive real-time, visual biofeedback in the clinical setting. The control group receives care as usual and the intervention group receives care as usual with the addition of real-time, visual biofeedback to improve weight bearing to the optimal level. Hypothetically, patients in the intervention group show (1) an increase in maximum peak loads and (2) faster step durations at an earlier timepoint in their rehabilitation. Furthermore, patients in the intervention group recover faster, which results in an earlier discharge date.

\section{Methods}

Study design

This protocol is described according to the SPIRIT (Standard Protocol Items: Recommendations for Interventional Trials) Statement. The study is an international, multicenter trial conducted between June 2017 and August 2018 in the Netherlands and Sweden. Rehabilitation centers focused on geriatric rehabilitation are asked to participate in this study. In order to ensure the quality and expertise of the participating centers, a minimum inclusion rate of at least 20 patients per year is expected. Therefore the number of eligible patients is screened in each center prior to their participation in the study. Measurements will be performed by trained physical therapists at the site and all physical therapists are experienced in geriatric trauma care. Prior to start of the study, all physical therapists agreed on the study treatment regime during multiple consensus meetings and were trained to uniformly implement this study treatment. Participating geriatric rehabilitation clinics are listed in Table 1.

This trial is a two-arm, parallel-design, prospective RCT. Based on a previous pilot study, the average length of rehabilitation is 44 days, with a standard deviation of 17 . Using a power of 0.80 , an alpha of $5 \%$ and an expected reduction of 7 days (one week) in two-sided testing, a sample size of 94 participants per group was calculated. Participants will be allocated to either the control group or the intervention group using a stratified cluster 


\section{Clinic}

Zorgspectrum

Geinsche Hof

Vuurscheschans 75

3432 TX Nieuwegein

\section{Warande}

Bovenwegen

Heideweg 2

3708 AT Zeist

\section{Warande}

Diakonessenhuis

Professor Lorentzlaan 76

3707 HL Zeist

Beweging 3.0

Meander Medical Center

Maatweg 3

3813 TZ Amersfoort

\begin{tabular}{|c|c|c|}
\hline $\begin{array}{l}\text { Evean } \\
\text { Schoenerstraat } 11 \\
1034 \text { XZ Amsterdam }\end{array}$ & Amsterdam / Zaandam (NL) & Intervention \\
\hline $\begin{array}{l}\text { Beweging } 3.0 \\
\text { Woonzorgcentrum De Pol } \\
\text { Vetkamp } 85 \\
3862 \text { JN Nijkerk }\end{array}$ & Nijkerk (NL) & Control \\
\hline $\begin{array}{l}\text { Cordaan } \\
\text { In het Zomerpark } \\
\text { Remmersteinpark 3-5 } \\
2151 \text { KE Nieuw-Vennep }\end{array}$ & Nieuw-Vennep (NL) & Control \\
\hline $\begin{array}{l}\text { azM Herstelzorg } \\
\text { Sint Pieterstraat } 23 \\
6211 \text { JM Maastricht }\end{array}$ & Maastricht (NL) & Control \\
\hline $\begin{array}{l}\text { Zorggroep Groningen } \\
\text { Schaaksport 100-102 } \\
9728 \text { PG Groningen }\end{array}$ & Groningen (NL) & Intervention \\
\hline $\begin{array}{l}\text { Careyn } \\
\text { Nieuw Tamarinde } \\
\text { Neckardreef } 6 \\
3562 \text { CN Utrecht }\end{array}$ & Utrecht (NL) & Control \\
\hline $\begin{array}{l}\text { Telge Rehab } \\
\text { Östra Kanalgatan } 2 \\
15271 \text { Södertälje }\end{array}$ & Södertälje (SE) & Control \\
\hline
\end{tabular}

Table 1. Participating geriatric rehabilitation clinics in The Netherlands and Sweden. Clinics were allocated to either the control or the intervention group.
Intervention

Intervention

Intervention

Amersfoort (NL) $\quad$ Control

(

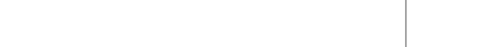


randomization according to the sealed opaque envelope principle. First, all rehabilitation clinics were divided into two groups depending on the inclusion rate: $<2$ vs. $\geq 2$ eligible participants per month. This division was made to avoid skewing of inclusion rate in both arms. Then in both groups, clinics were randomized by the principal investigator into the control or intervention group using the sealed opaque envelope principle. This resulted in six rehabilitation clinics in the control group and five rehabilitation clinics in the intervention group (Table 1). Randomization per center, or cluster randomization, was chosen for two reasons. First, individual selection bias by the physical therapist is minimized. Second, and more importantly, the physical therapists and participants within one center can now provide a single treatment, either care as usual or care as usual with real-time, visual biofeedback. Considering the constant interaction between physical therapists, as well as between participants, individual randomization would increase the risk of data collection errors.

\section{Participants}

Strict inclusion criteria will be used to introduce homogeneity of the groups, and therefore, participants enrolled in the study meet the following inclusion criteria:

- The participants rehabilitate from a proximal femur fracture following low energy trauma (e.g. fall from standing position).

- The participants have a prescribed unrestricted weight bearing after (surgical) treatment of their fracture.

- The participants have an expected clinical rehabilitation duration of $\geq 2$ weeks.

- The participants are $\geq 60$ years.

- The participants have a bodyweight $\leq 120$ kilogram.

Participants with the following criteria will be excluded from the study:

- People with cognitive impairment, defined as a score $<18$ on the Mini-Mental State Examination [16].

- People who are readmitted to the hospital within two weeks after study participation, for example for infectious complications.

- People with co-morbidities that affected gait significantly prior to the proximal femur fracture. 


\section{Protocol}

Data will be gathered according to a strict study protocol (Fig 1). Candidate patients who fulfill the inclusion criteria will be asked to participate in the study by the site investigator and receive an information letter with the study protocol and study objectives. If patients agree to participate in the study, informed consent will be signed and the cognitive score will be evaluated by the Mini Mental State Examination (MMSE). The MMSE is an objective score to quantitatively asses the severity of cognitive impairment. A MMSE score $<18$ indicates severe cognitive impairment [16] and this leads to exclusion of the patient in this trial. After informed consent and MMSE, data will be collected in participants according to the following trial protocol. The institutional protocol for physical therapy after proximal femur fractures will be followed in all participants. In addition, each participant receives daily force measurements using the SensiStep system in a 30-meter walk to measure the gait parameters. Additional clinical tests will be executed to gain additional insight in the rehabilitation progress of each participant:

- Functional Ambulation Categories (FAC, once per week)

- Elderly Mobility Scale (EMS, twice per week)

- Visual Analogue Scale (VAS, daily)

Finally, additional information of each participant will be documented, including the following:

- Date of surgery

- Type of surgery

- Co-morbidities

- Walking aid

- Length of admission to the rehabilitation center

- Age

- Gender

- Length

- Weight 


\begin{tabular}{|c|c|c|c|c|c|c|c|c|}
\hline & \multicolumn{8}{|c|}{ STUDY PERIOD } \\
\hline & \multirow{2}{*}{$\begin{array}{c}\text { Enrolment } \\
-t_{1}\end{array}$} & \multirow{2}{*}{$\begin{array}{c}\text { Allocation } \\
t_{0} \text { (start) }\end{array}$} & \multicolumn{5}{|c|}{ Post-allocation } & \multirow{2}{*}{$\begin{array}{l}\text { Close-ou } \\
t_{x}(e n d)\end{array}$} \\
\hline TIMEPOINT & & & $t_{1}$ & $t_{2}$ & $t_{3}$ & $t_{4}$ & etc. & \\
\hline \multicolumn{9}{|l|}{ ENROLMENT: } \\
\hline Eligibility screen & $\mathrm{X}$ & & & & & & & \\
\hline Informed consent & $\mathrm{X}$ & & & & & & & \\
\hline MMSE & $\mathrm{X}$ & & & & & & & \\
\hline Allocation & & $\mathrm{X}$ & & & & & & \\
\hline \multicolumn{9}{|l|}{ INTERVENTIONS: } \\
\hline \multicolumn{9}{|l|}{$\begin{array}{l}\text { SensiStep measurement } \\
\text { without feedback } \\
\text { (Control group) }\end{array}$} \\
\hline \multicolumn{9}{|l|}{$\begin{array}{c}\text { SensiStep measurement } \\
\text { with feedback }\end{array}$} \\
\hline \multicolumn{9}{|l|}{ (Intervention group) } \\
\hline \multicolumn{9}{|l|}{ ASSESSMENTS: } \\
\hline \multicolumn{9}{|l|}{ EMS } \\
\hline \multicolumn{9}{|l|}{$F A C$} \\
\hline \multicolumn{9}{|l|}{$V A S$ (pain) } \\
\hline \multicolumn{9}{|l|}{ DOCUMENTATION: } \\
\hline $\begin{array}{l}\text { Note patient } \\
\text { demographics }\end{array}$ & & $\mathrm{X}$ & & & & & & \\
\hline Note date of discharge & & & & & & & & $\mathrm{X}$ \\
\hline
\end{tabular}

Fig 1. Schedule of enrolment, interventions, assessments and documentation. MMSE Mini Mental State Examination, EMS Elderly Mobility Scale, FAC Functional Ambulation Categories, VAS Visual Analogue Scale. 


\section{Interventions}

Participants in the intervention group receive, in addition to the standard institutional protocol, real-time visual feedback about weight bearing during the 30-meter walk with SensiStep. The physical therapist has real-time visual insight in the weight bearing as well by observing the tablet together with the participant. Both the biofeedback system (visually) and the physical therapist (verbally) assist and motivate the participant to adapt the weight bearing to the optimal level. The target weight will be set at $100 \%$ bodyweight, as the participants have no restrictions in weight bearing.

\section{Outcome measures}

The primary outcome measures are the gait parameters maximum peak load (in \%bodyweight) and step duration (in seconds), which will be analyzed in relation to the discharge date from the rehabilitation center. It is expected that the intervention group will show an improvement in gait parameters and earlier discharge date compared to the control group. The discharge date will be determined by a multidisciplinary team within the usual institutional regime, irrespective of the study protocol. Secondary outcomes include other spatio-temporal gait parameters and validated clinical tests, including the Elderly Mobility Scale (EMS), the Functional Ambulation Categories (FAC) and the Visual Analogue Scale (VAS). The EMS is a standardized validated scale for assessment of frail elderly people. The FAC is used as a diagnostic and evaluative tool to assess the physical performance of patients. The VAS is a commonly used, subjective scale to assess the amount of pain patients experience. Spatio-temporal gait parameters measured by the SensiStep will be correlated to the clinical test scores (e.g. EMS, FAC and VAS). Blinding the participants and/or physical therapist is not possible, as both users are aware of the biofeedback they receive in the intervention group. However, data will be encrypted and anonymously delivered by the Steering Committee to the data scientists, which makes the data scientists blinded for the analysis of gathered data.

\section{Statistical analysis}

Statistical analysis on outcome measures will be conducted using Statistical Package for the Social Sciences (SPSS). First, data distribution will be assessed. Second, missing data will be handled by a multiple imputation technique. The primary analysis will be based on an intention-to-treat principle. Also, a per-protocol analysis will be performed as sensitivity analysis to assess the robustness of the results to protocol deviations [17]. The primary goal 
is to find a relation between the gait parameters (maximum peak load and step duration) and the discharge date. In addition, other spatio-temporal gait parameters measured by the SensiStep will be analyzed for this relation as well. The secondary goal is to find a relation between the gait parameters and the clinical measurements (i.e. EMS, FAC and VAS). Relations between all data will be examined using multiple regression analysis.

\section{Monitoring}

This trial will be conducted according to the principles of the Declaration of Helsinki (amended version by the 64th WMA General Assembly, Fortaleza, Brazil, October 2013) and in accordance with the Medical Research Involving Human Subjects Act. Gathered data will be encrypted and stored on a secure server and is accessible by the Steering Committee.

This trial will be conducted under strict supervision of an experienced Steering Committee from the University Medical Center Utrecht (UMCU), Maastricht University Medical Center+ (MUMC+) and Uppsala University (UU). Acting as a Steering Committee, the principal investigators from UMCU and MUMC+ will discuss the progress of enrollment at least once every two weeks. The Steering Committee will perform a blinded interim analysis on $30 \%, 60 \%$ and $90 \%$ of the included participants. During these interim analysis, reasons for exclusion will be discussed with the site investigators. Other items will be discussed as well, including but not limited to, applied discharge criteria for each patient, adverse events, quality of the retrieved data, missing data, and necessity to include other participating centers.

A monthly newsletter will be written by the Steering Committee and distributed among the participating centers. In this newsletter the inclusion rate will be shown per center, which is a common and effective way to increase awareness and readiness to include patients in multi-center trials. The trial progress and general results will be communicated by the Steering Committee upon request.

\section{Discussion}

The worldwide incidence of hip fractures among elderly will increase due to the ageing population [1]. As hip fractures have a high morbidity and mortality, improvements should be made in the treatment of hip fractures. Biofeedback system have previously shown their potential, as weight bearing significantly improved in partial and full weight bearing [9]. 
This could improve fracture healing through mechanotransduction [6]. As stated before, the hypothesis is that real-time visual biofeedback results in a significant improvement in weight bearing in elderly after proximal femur fractures, especially with respect to the gait parameters maximum peak load and step duration, compared to standard training methods. Secondly, it is hypothesized that improvements in spatio-temporal gait parameters will lead to improvements in clinical scores, such as the EMS, FAC and VAS. The aim of our trial is to validate these hypotheses and investigate the effect of real-time, visual biofeedback on weight bearing during the rehabilitation after proximal femur fractures in elderly. This potentially leads to improved rehabilitation (e.g. better functional outcome), faster rehabilitation and lower medical costs.

Although extensive attention has been paid to the study design, some difficulties still remain. One of the difficulties in this trial is the study population. Often, elderly who sustain a hip fracture due to low energy trauma have (multiple) co-morbidities that could negatively affect gait. It is difficult to detect the amount of influence of these co-morbidities on gait. The question arises if any improvements or deterioration in gait is caused by realtime visual biofeedback or by the co-morbidities. To overcome this issue, the strict inclusion criteria for participants should minimize variability in the data. Moreover, the large number of participants (e.g. 94 per arm) should minimize the effect of co-morbidities on gait.

The topic of adherence could be another issue in this trial, as the trial will cause additional burden to both the participants and physical therapists. It is therefore important to select enthusiastic and experienced rehabilitation centers as candidate centers. This will be determined by asking candidate centers to register all eligible patients before the start of this trial during two months. Furthermore, the Steering Committee will weekly evaluate the inclusion rate and search for missing data, which provides the opportunity to steer directly if any issues are detected.

The potential effect of real-time, visual biofeedback on weight bearing can have major influence on rehabilitation after proximal femur fractures in elderly, and therefore, dissemination is important. A stakeholder analysis will be performed early in the project, and determine how dissemination to these stakeholders should be handled in order to convince them of the added value of real-time, visual biofeedback. The stakeholder analysis will identify which organizations and people the dissemination should target. 
Dissemination will take place at different levels, most important the patient level, the care provider level, and the level of the healthcare organizations, including insurance companies.

In summary, this is a multicenter RCT to investigate the rehabilitation progress and the value of real-time visual biofeedback on weight bearing during rehabilitation after proximal femur fractures in elderly. Primary focus lies on the gait parameters maximum peak load and step duration in relation to discharge date, however, secondary outcomes in other spatio-temporal gait parameters and additional validated clinical tests will be analyzed as well. This trial will contribute to existing knowledge of rehabilitation after hip fractures in elderly, hopefully contributing to improved outcome for many affected. 


\section{References}

[1] Dhanwal D, Dennison E, Harvey N, Cooper C. Epidemiology of hip fracture: Worldwide geographic variation. Indian J Orthop. 2011;45:15-22.

[2] Peeters CMM, Visser E, Van De Ree CLP, Gosens T, Den Oudsten BL, De Vries J. Quality of life after hip fracture in the elderly: A systematic literature review. Injury. 2016;47:1369-82. doi:10.1016/j.injury.2016.04.018.

[3] Beaupre LA, Binder EF, Cameron ID, Jones CA, Orwig D, Sherrington C, et al. Maximising functional recovery following hip fracture in frail seniors. Best Pract Res Clin Rheumatol. 2013;27:771-88. doi:10.1016/j.berh.2014.01.001.

[4] Vasarhelyi A, Baumert T, Fritsch C, Hopfenmüller W, Gradl G, Mittlmeier T. Partial weight bearing after surgery for fractures of the lower extremity - is it achievable? Gait Posture. 2006;23:99-105. doi:10.1016/j.gaitpost.2004.12.005.

[5] Robling AG, Castillo AB, Turner CH. Biomechanical and Molecular Regulation of Bone Remodeling. Annu Rev Biomed Eng. 2006;8:455-98.

[6] Klein-Nulend J, Bacabac RG, Mullender MG. Mechanobiology of bone tissue. Pathol Biol. 2005;53:576-80.

[7] Hurkmans HLP, Bussmann JBJ, Benda E, Verhaar J a N, Stam HJ. Techniques for measuring weight bearing during standing and walking. Clin Biomech. 2003;18:576-89.

[8] Hershko E, Tauber C, Carmeli E. Biofeedback Versus Physiotherapy in Patients With Partial Weight-Bearing. Am J Orthop. 2008;37:92-6.

[9] Raaben M, Holtslag HR, Leenen LPH, Augustine R, Blokhuis TJ. Real-time visual biofeedback during weight bearing improves therapy compliance in patients following lower extremity fractures. Gait Posture. 2018;59 October 2017:206-10. doi:10.1016/j.gaitpost.2017.10.022.

[10] Jiang Y, Norman KE. Effects of visual and auditory cues on gait initiation in people with Parkinson's disease. Clin Rehabil. 2006;20:36-45. doi:10.1191/0269215506cr925oa.

[11] Druzbicki M, Guzik A, Przysada G, Kwolek A, Brzozowska-Magoń A. Efficacy of gait training using a treadmill with and without visual biofeedback in patients after stroke: A randomized study. J Rehabil Med. 2015;47:41925 .

[12] Baram Y, Lenger R. Gait improvement in patients with cerebral palsy by visual and auditory feedback. Neuromodulation. 2012;15:48-52.

[13] Isakov E. Gait rehabilitation: a new biofeedback device for monitoring and enhancing weight-bearing over the affected lower limb. Eura Medicophys. 2007;43:21-6.

[14] Hurkmans HL, Bussmann JB, Benda E, Verhaar JA, Stam HJ. Effectiveness of audio feedback for partial weight-bearing in and outside the hospital: A randomized controlled trial. Arch Phys Med Rehabil. 2012;93:565-70. doi:10.1016/j.apmr.2011.11.019.

[15] Raaben M, Holtslag H, Augustine R, van Merkerk R, Koopman B, Blokhuis T. Technical Aspects and Validation of a New Biofeedback System for Measuring Lower Limb Loading in the Dynamic Situation. Sensors. 2017;17:658. doi:10.3390/s17030658. 
[16] Tombaugh TN, McIntyre NJ. The mini-mental state examination: a comprehensive review. Journal of the American Geriatrics Society. 1992;40:922-35.

[17] Thabane L, Mbuagbaw L, Zhang S, Samaan Z, Marcucci M, Ye C, et al. A tutorial on sensitivity analyses in clinical trials: The what, why, when and how. BMC Med Res Methodol. 2013;13:1. doi:10.1186/1471-2288-13-92. 
CHAPTER
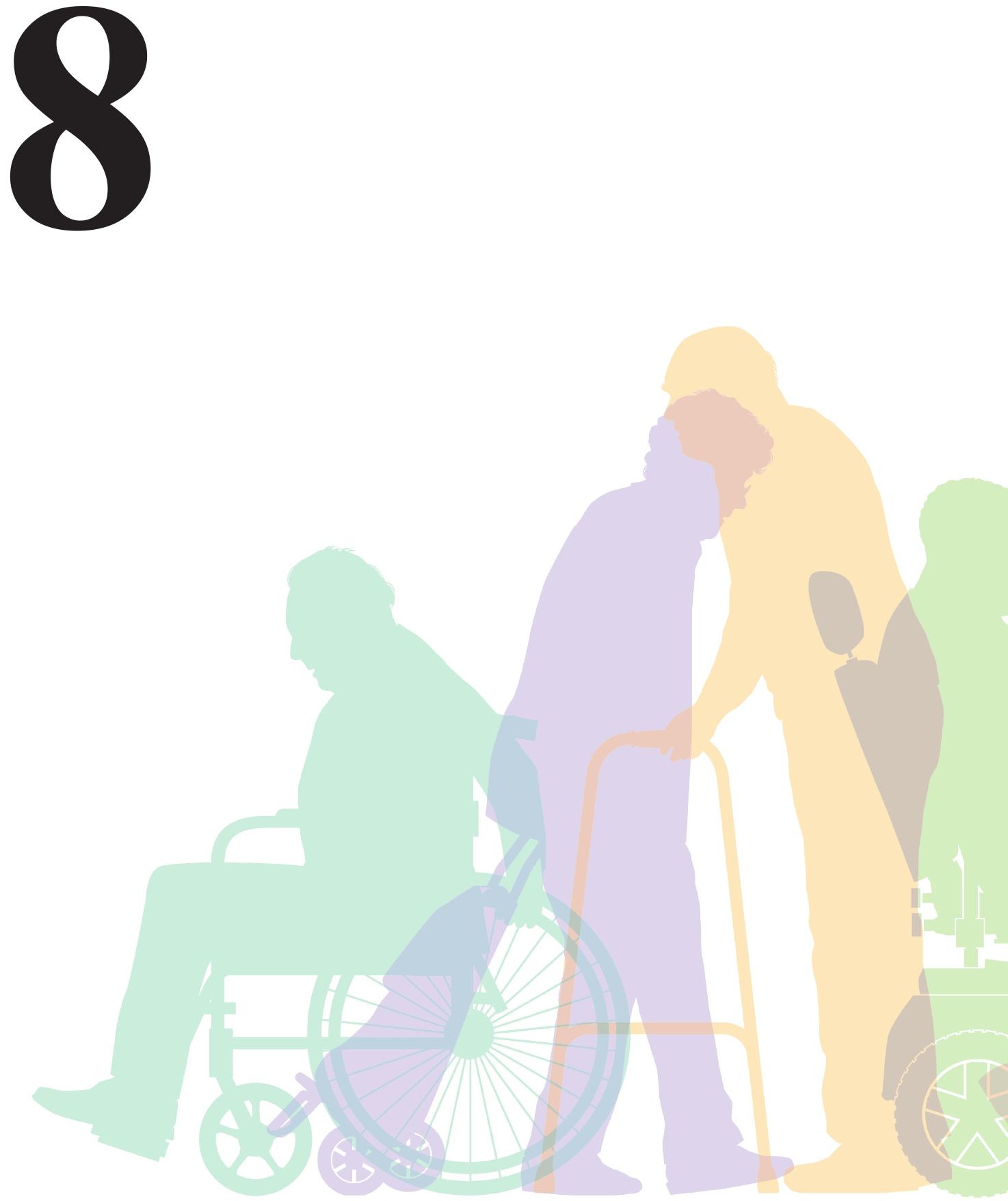
Real-time biofeedback improves rehabilitation after hip fractures in elderly: an international, multicenter randomised controlled trial (COMFORT)

Submitted for publication

M. Raaben, S. Redzwan, R. Augustine, T.J. Blokhuis 


\begin{abstract}
Background: Biofeedback systems have shown their added value in rehabilitation after lower extremity fractures. Important gait parameters and the rehabilitation progress could be monitored in elderly patients following a hip fracture. The current RCT aimed to answer the question if these gait parameters and rehabilitation progress could be improved by giving real-time biofeedback to these frail patients.
\end{abstract}

Materials and Methods: Patients were included in geriatric rehabilitation centers in The Netherlands and Sweden if they met the following inclusion criteria, (1) patients following a proximal femur fracture, (2) prescribed unrestricted weight bearing, (3) expected clinical rehabilitation of $\geq 2$ weeks, (4) age $\geq 60$ years and (5) body weight $\leq 120 \mathrm{~kg}$. Patients with (1) a MMSE score $\leq 18$, (2) hospital readmission within 2 weeks and (3) pre-existent comorbidities affecting gait were excluded. The participants were randomised into either the control group, which received care as usual, or the intervention group, were real-time biofeedback by SensiStep was given to change gait patterns. Primary gait parameters of interest were maximum peak load and step duration. Other gait parameters were assessed as well and the results were compared to the EMS, FAC and VAS score.

Results: 115 participants were included between June 2017 and August 2018. 60 participants were randomised into the control group and 55 participants into the intervention group. Both groups showed significant improvements in all gait parameters between the start and end of rehabilitation. Significant higher improvements in maximum peak load were found in the intervention group. Moreover, a higher effect of biofeedback was shown in particular, previously defined, subpopulations of the participants. The results in other gait parameters and the EMS, FAC and VAS score were similar in both groups.

Discussion: The results in current RCT emphasized the importance of real-time biofeedback about weight bearing in rehabilitation after hip fractures in elderly. The effect of biofeedback can be improved by developing parallel feedback mechanisms about other gait parameters. It is recommended to implement biofeedback systems in future rehabilitation programs to optimize gait and thereby fracture healing outcome after hip fractures in elderly.

Keywords: hip fracture, rehabilitation, gait analysis, elderly, biofeedback 


\section{Introduction}

Hip fractures in elderly are a major health concern with a high social and economic impact [1]. More than $30 \%$ of the patients will not survive the first year following the hip fracture [2]. The surviving patients experience significant limitations in mobility and a reduced quality of life [3]. Improvement of this situation requires optimization of the care for these fragile patients, including optimal medical care, optimal fracture treatment and state-of-theart rehabilitation programs. Large clinical trials have been performed to investigate the optimal medical and fracture treatment [4,5]. Unfortunately, the opposite is true for rehabilitations programs and these are therefore not yet available.

The added value of proper rehabilitation, and especially resumption of weight bearing, is clear. It is known for example that early weight bearing has many beneficial effects on fracture healing outcome, including improved coordination, muscle mass and bone remodelling through mechanotransduction [6]. It is therefore remarkable that rehabilitation in orthopaedic trauma care currently uses out-dated techniques, such as bathroom scales, with severe limitations in their application and reliability. The main drawback is that bathroom scales are only able to provide insight in weight bearing in the static situation. Important information is lost in the dynamic situation, leading to low therapy compliance by patients in the ambulatory, clinical situation [7-10]. Previous studies have shown the potential of biofeedback systems to improve therapy compliance and resumption of weight bearing in various conditions, including lower extremity fractures [11-14]. Although biofeedback systems and their beneficial effects on therapy compliance are gaining attention in literature, these systems are not yet incorporated in current rehabilitation programs. In fact, the gold standard still relies on subjective mobility scores as the Functional Ambulation Categories (FAC) and Elderly Mobility Scale (EMS) [15].

It was previously demonstrated that rehabilitation progress after hip fractures in elderly can be quantified with the biofeedback system SensiStep (Evalan BV, Amsterdam, the Netherlands) [14]. Especially, the gait parameters maximum peak load and step duration were considered important, as these parameters were related to the day of discharge. The endpoint of rehabilitation could objectively be defined by monitoring these two gait parameters instead of using the subjective FAC and EMS scores [14]. The question remained, however, if real time biofeedback about gait parameters could also improve the rehabilitation progress and thereby fracture healing outcome after hip fractures in the 
elderly. Therefore, the aim of current randomised controlled trial was to investigate whether elderly patients recover faster or better after a hip fracture if real-time biofeedback was provided by SensiStep, compared to patients that received care as usual. The hypothesis was that patients in the intervention group would show (1) an increase in maximum peak load and (2) faster steps compared to the control group. Also, it was expected that patients in the intervention group would rehabilitate faster and reach the objective endpoint of rehabilitation earlier. Hypothetically, this also would result in an earlier discharge date. The results were compared to the gold standard mobility tests FAC and EMS.

\section{Materials and Methods}

\section{Geriatric rehabilitation clinics}

The current study is an international, multicenter study conducted between June 2017 and August 2018 in The Netherlands and Sweden. Eligible geriatric rehabilitation clinics were asked to participate in the study. To ensure adequate exposure and routine of the participating personnel and clinics, a minimum expected inclusion rate of 20 patients per year was required. All physical therapists agreed on the study protocol as described below and were trained during multiple consensus meetings before the study start date. Study measurements were performed on site by experienced physical therapists with specific focus on geriatric trauma care. Eligible patients were informed about the study at the time of admission to the rehabilitation clinic. If they were interested in participation, they were screened according to the inclusion and exclusion criteria as described below and informed consent was signed by the patient and the physical therapist at the same moment. This research protocol was approved by the accredited Ethics Committee Slotervaart Hospital and Reade (number P1658).

\section{Inclusion and exclusion criteria}

Participants that were recruited for this study were all admitted to clinical geriatric rehabilitation. Admission to this facility requires patients to meet all criteria that are described in the Dutch triage protocol for geriatric rehabilitation ('Triage Instrument Geriatrische Revalidatiezorg') [16]. Geriatric rehabilitation differs from medical rehabilitation. In short, to meet the criteria for geriatric rehabilitation patients have to suffer from one or multiple diseases that result in permanent limitations and they need more help than basic monodisciplinary treatment to overcome these limitations. The motor and cognitive functioning, communication ability and personal factors are assessed, together 
defined as the medical stability. In addition to the medical stability, therapy intensity and motivation are assessed during the triage for geriatric rehabilitation. The medical stable, high intensity and motivated patients are selected for medical rehabilitation, while the medical unstable, low intensity and unmotivated patients are selected for clinical geriatric rehabilitation. One important aspect for clinical geriatric rehabilitation is that the patients' motivation could be improved by therapy [16].

In addition to the criteria from the triage instrument, patients that were recruited for the current study met the following inclusion criteria to ensure homogeneity of the groups:

- Admission following a proximal femur fracture (AO/OTA type 31A-B) resulting from low energy trauma

- Prescribed unrestricted weight bearing after surgical treatment of the fracture

- An expected clinical rehabilitation of $\geq 2$ weeks

- Age $\geq 60$ years

- Body weight $\leq 120 \mathrm{~kg}$

Patients that met the following exclusion criteria were excluded from the study:

- Cognitive impairment, defined as a Mini Mental State Examination (MMSE) score $\leq 18$

- Hospital readmission within 2 weeks after study participation, for example for infectious complications or failure of the fracture fixation

- Pre-existent co-morbidities that significantly affected gait prior to the proximal femur fracture

\section{Randomisation}

The current study is a two-arm, parallel-design, prospective randomised controlled trial. Randomisation was done by stratified cluster randomisation. First, all rehabilitation clinics were divided into two groups depending on their expected inclusion rate: $<2 \mathrm{vs}$. $\geq 2$ eligible participants per month. This was chosen to avoid skewing of the inclusion rate in either group. Second, the clinics in both groups (i.e. high or low inclusion rate) were randomised into either the control group or the intervention group. Randomisation per clinic was chosen for two reasons. First, individual selection bias by the treating physical therapist is avoided. Second, a single treatment (i.e. feedback or no feedback) could be given to the participants. 
Considering the constant interaction between physical therapists and participants as well as the interaction between participants, individual randomisation would increase the risk of data collection errors or cross-over effects. The randomisation procedure resulted in six rehabilitation clinics in the control group and five rehabilitation clinics in the intervention group (Table 1).

\section{Biofeedback system}

The ambulatory biofeedback system SensiStep was used to measure gait patterns during rehabilitation after proximal femur fractures in elderly. SensiStep was previously validated and is able to measure gait parameters reliably and continuously in the clinical setting [17]. Moreover, SensiStep is able to provide real-time biofeedback about weight bearing to both the patient and healthcare professional, offering the opportunity to directly adapt weight bearing to the optimal level. The feedback is given using a graphic representation of the steps on a tablet screen. The pressure curve of each step is shown in real-time and represented as percentage of the body weight of the patient. The target weight, including a range, is set prior to the session and shown as a green bar on the screen.

\section{Study protocol}

The gait of the participants, both in the intervention and in the control group, was measured daily with the SensiStep system in a 30 meter walk. The control group received care as usual and SensiStep was only used to measure gait parameters. In addition to care as usual, the intervention group received real-time insight in their weight bearing, enabling them to adapt the weight bearing to the optimal level. Also, the physical therapist had real-time insight in weight bearing, enabling them to verbally assist and motivate the participants in the intervention group to improve their weight bearing. The target weight for all participants was set at $100 \%$ body weight $(\mathrm{BW})$. The desired range was $+/-10 \% \mathrm{BW}$. In both groups mobility tests were obtained weekly, including the Elderly Mobility Scale (EMS), Functional Ambulation Categories (FAC) and Visual Analogue Scale (VAS) pain score in order to compare the rehabilitation progress measured by SensiStep with previously validated clinical tests. 


\begin{tabular}{|c|c|c|}
\hline Clinic & Place & Allocation (group) \\
\hline $\begin{array}{l}\text { Zorgspectrum } \\
\text { Geinsche Hof } \\
\text { Vuurscheschans } 75 \\
3432 \text { TX Nieuwegein }\end{array}$ & Nieuwegein (NL) & Intervention \\
\hline $\begin{array}{l}\text { Warande } \\
\text { Bovenwegen } \\
\text { Heideweg } 2 \\
3708 \text { AT Zeist }\end{array}$ & Zeist (NL) & Intervention \\
\hline $\begin{array}{l}\text { Warande } \\
\text { Diakonessenhuis } \\
\text { Professor Lorentzlaan } 76 \\
3707 \text { HL Zeist }\end{array}$ & Zeist (NL) & Intervention \\
\hline $\begin{array}{l}\text { Beweging 3.0 } \\
\text { Meander Medical Center } \\
\text { Maatweg } 3 \\
3813 \text { TZ Amersfoort }\end{array}$ & Amersfoort (NL) & Control \\
\hline $\begin{array}{l}\text { Evean } \\
\text { Schoenerstraat } 11 \\
1034 \text { XZ Amsterdam }\end{array}$ & Amsterdam / Zaandam (NL) & Intervention \\
\hline $\begin{array}{l}\text { Beweging 3.0 } \\
\text { Woonzorgcentrum De Pol } \\
\text { Vetkamp } 85 \\
3862 \text { JN Nijkerk }\end{array}$ & Nijkerk (NL) & Control \\
\hline $\begin{array}{l}\text { Cordaan } \\
\text { In het Zomerpark } \\
\text { Remmersteinpark 3-5 } \\
2151 \text { KE Nieuw-Vennep }\end{array}$ & Nieuw-Vennep (NL) & Control \\
\hline $\begin{array}{l}\text { azM Herstelzorg } \\
\text { Sint Pieterstraat } 23 \\
6211 \text { JM Maastricht }\end{array}$ & Maastricht (NL) & Control \\
\hline $\begin{array}{l}\text { Zorggroep Groningen } \\
\text { Schaaksport 100-102 } \\
9728 \text { PG Groningen }\end{array}$ & Groningen (NL) & Intervention \\
\hline $\begin{array}{l}\text { Careyn } \\
\text { Nieuw Tamarinde } \\
\text { Neckardreef } 6 \\
3562 \text { CN Utrecht }\end{array}$ & Utrecht (NL) & Control \\
\hline $\begin{array}{l}\text { Telge Rehab } \\
\text { Östra Kanalgatan } 2 \\
15271 \text { Södertälje }\end{array}$ & Södertälje (SE) & Control \\
\hline
\end{tabular}

Table 1. Participating geriatric rehabilitation clinics in the COMFORT study. All clinics had experience with and focus on geriatric patients. Patients were recruited in rehabilitations clinics located in the Netherlands and Sweden. 


\section{Interim analysis}

As described previously, the total sample size of 94 participants per group was calculated, and an interim analysis was planned on 30\%, 60\% and $90 \%$ of the foreseen number of inclusions [18]. At the second interim analysis (i.e. 60\%), significant differences were found between the intervention and control group. For ethical considerations and to prevent unnecessary measurements, the Steering Committee decided to stop new inclusions for this study. At this point, a total of 115 patients were included in the study.

\section{Statistical analysis}

Statistical analysis was conducted using Graphpad Prism V6.01 (GraphPad Software, La Jolla, USA). First, a D'Agostino \& Pearson omnibus K2 normality test was done to analyse for Gaussian distribution of the dataset. A Student $\mathrm{T}$ test was done to analyse for significance if the data has a Gaussian distribution. If there was no Gaussian distribution, then a Mann-Whitney test was done to assess whether differences between both groups were significant. Significance was set at $\alpha=5 \%$.

\section{Results}

A total of 130 consecutive elderly patients rehabilitating from a hip fracture were asked to participate in this study between June 2017 and August 2018. 15 patients were excluded due to cognitive deterioration (1), hospitalization (4) and $<2$ weeks of rehabilitation (10). A total of 115 patients participated in the current study. Of these, 60 participants were randomised into the control group and 55 participants were randomised into the intervention group. The participants in the control group $(13 \hat{\partial}, 47+)$ had a mean age of $82.2 \pm 10.6$ years and mean BMI of $24.6 \pm 4.48$. The participants in the intervention group

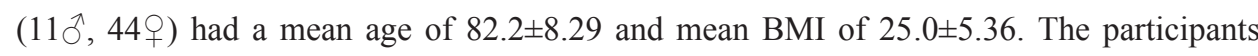
entered in the study protocol at the time of admission in the geriatric rehabilitation institution. In the control and intervention group this was respectively 16.0 99.07 and $16.6 \pm 11.1$ days after surgery. The participants underwent different surgical procedures following their hip fracture and, as expected, most participants suffered from (multiple) comorbidities. The details are summarized in Table 2.

Total admission time was comparable for the control and intervention group, respectively $28.4 \pm 15.2$ and $31.5 \pm 15.3$ days. Significant improvement in both gait parameters peak load and step duration was seen in both groups after finishing the rehabilitation period. For peak load, the control group started with a mean maximum peak load of $59.76 \pm 17.91 \% \mathrm{BW}$ 
during the first training session. A significant improvement to $70.00 \pm 16.30 \% \mathrm{BW}$ $(\mathrm{P}=0.0014)$ was found at the day of discharge during the last training session. Patients in the intervention group started on average with a mean maximum peak load of $53.41 \pm 19.88 \% \mathrm{BW}$ in the first training session.

\begin{tabular}{|c|c|c|}
\hline & Control group $(n=60)$ & Intervention group $(n=55)$ \\
\hline \multicolumn{3}{|c|}{ Demographics } \\
\hline Age (years) & $82.2 \pm 10.6$ & $82.2 \pm 8.29$ \\
\hline Sex (male:female) & $13: 47$ & $11: 44$ \\
\hline Height (cm) & $166.4 \pm 7.53$ & $165.7 \pm 10.0$ \\
\hline Weight (kg) & $68.1 \pm 13.2$ & $67.3 \pm 14.0$ \\
\hline BMI & $24.6 \pm 4.48$ & $25.0 \pm 5.36$ \\
\hline MMSE & $26.4 \pm 3.61$ & $25.5 \pm 3.33$ \\
\hline \multicolumn{3}{|c|}{ Comorbidity } \\
\hline None & 12 & 9 \\
\hline Cardiovascular & 4 & 12 \\
\hline Musculoskeletal & 7 & 9 \\
\hline Neurological & 4 & 5 \\
\hline Respiratory & 9 & 7 \\
\hline Multiple & 24 & 13 \\
\hline \multicolumn{3}{|c|}{ Surgery } \\
\hline THA/HHA & 32 & 17 \\
\hline DHS & 2 & 7 \\
\hline Gamma Nail & 7 & 8 \\
\hline Cannulated Screws & 1 & 0 \\
\hline PFN & 12 & 18 \\
\hline Other & 6 & 5 \\
\hline
\end{tabular}

Table 2. Characteristics of patients in both groups. BMI=Body Mass Index, MMSE=Mini Mental State Examination, THA=Total Hip Arthroplasty, HHA=Hemi Hip Arthroplasty, DHS=Dynamic Hip Screw, PFN=Pertrochanteric Femur Nail 
Similar to the control group, a significant improvement in maximum peak load was found at the day of discharge to $73.74 \pm 14.86 \%$ BW $(\mathrm{P}<0.0001)$. The improvement in peak load was significantly higher in the intervention group compared to the control group (20.33 $\pm 17.55 \%$ BW versus $10.24 \pm 14.97 \%$ BW, respectively, $\mathrm{P}=0.0012)$. Also, $96.36 \%$ of the patients in the intervention group improved during rehabilitation, compared to $76.67 \%$ of the control group. These findings are also illustrated by regression analysis in Figure 1. It can be clearly seen that patients in the intervention group show higher improvements compared to patients who receive care as usual. Also, 14 patients $(23.3 \%)$ in the control group deteriorated during rehabilitation, versus 2 patients $(3.64 \%)$ in the intervention group. For step duration, the control group started with a step duration of $1.42 \pm 0.51 \mathrm{~s}$ and ended with $1.09 \pm 0.24 \mathrm{~s}$ at the day of discharge $(\mathrm{P}<0.0001)$. In the intervention group an improvement was found from $1.28 \pm 0.44 \mathrm{~s}$ at the start to $1.09 \pm 0.28 \mathrm{~s}$ at the end of rehabilitation $(\mathrm{P}=0.0080)$. The improvement in step duration was not significantly different between the control and intervention group $(\mathrm{P}=0.1766)$.

It was previously suggested that patients could be differentiated into four groups based on the first SensiStep session, i.e. patients that start with a (1) high peak load, fast step duration, (2) high peak load, slow step duration, (3) low peak load, fast step duration and (4) low peak load, slow step duration [14]. The current study especially showed a significant effect $(\mathrm{P}=0.0151)$ of biofeedback by SensiStep in groups 3 and 4, i.e. patients that start with low peak loads. The observed effect in the two groups that started rehabilitation with high peak loads was similar for control and intervention (i.e. group 1 and 2, $\mathrm{P}=0.3211$ ).

Significant improvements between the start and end of rehabilitation were also found for both groups in the mobility tests. For the control group, the EMS increased from 15.0 \pm 2.91 at the start to $17.6 \pm 1.87$ at the end, the FAC from $3.37 \pm 0.79$ at the start to $4.03 \pm 0.59$ at the end and the VAS decreased from $4.09 \pm 2.07$ at the start to $2.10 \pm 1.87$ at the end. Similar improvements were found for the intervention group, were the EMS increased from $14.8 \pm 3.88$ at the start to $17.5 \pm 1.93$ at the end, the FAC from $3.32 \pm 0.58$ at the start to $3.96 \pm 0.35$ at the end and the VAS decreased from $4.72 \pm 1.69$ at the start to $2.70 \pm 1.98$ at the end. The improvements were not significantly different between both groups for EMS score $(\mathrm{P}=0.1069)$, FAC score $(\mathrm{P}=0.9785)$ and VAS score $(\mathrm{P}=0.4363)$. Details and $\mathrm{p}$-values are shown in Table 3. 


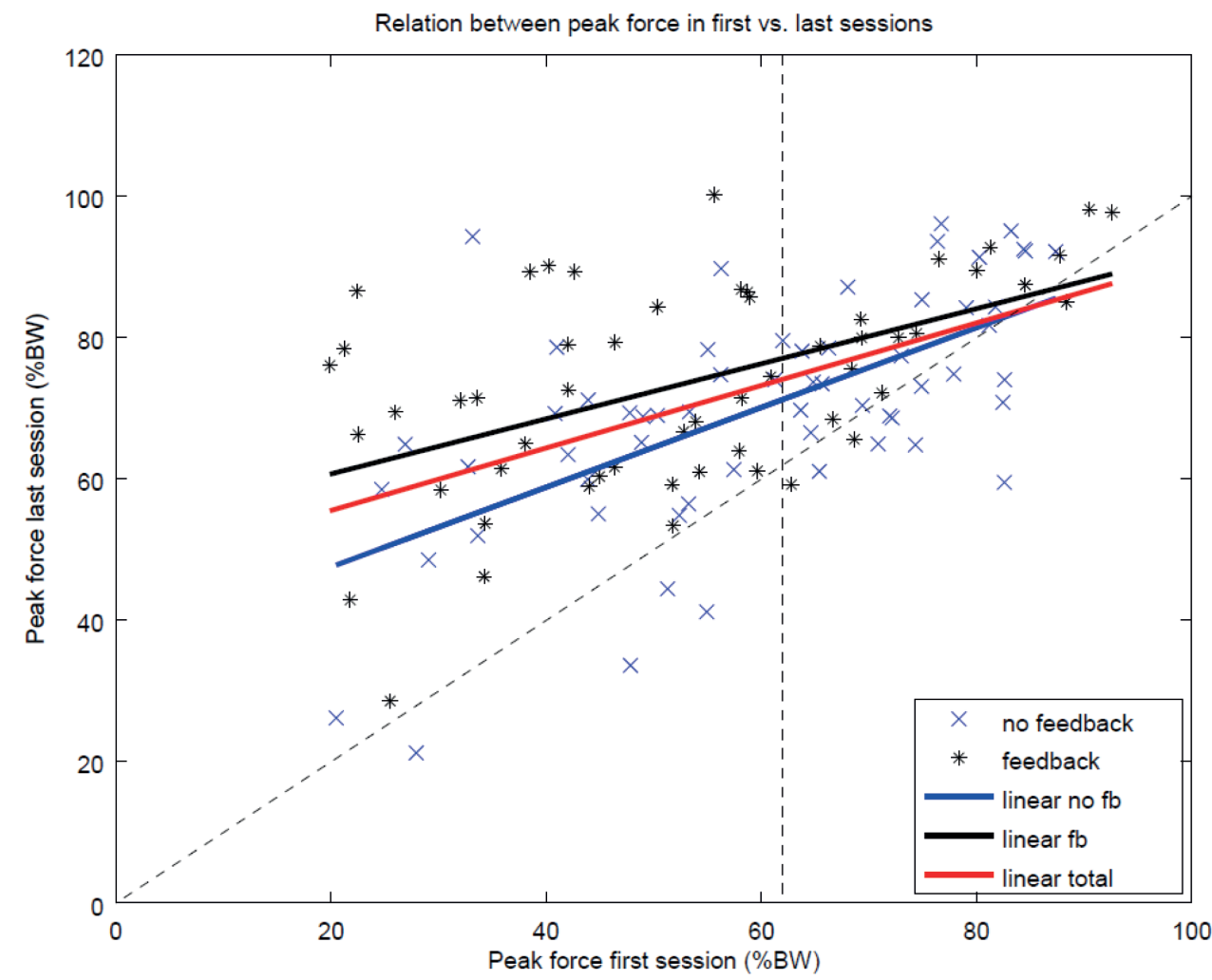

Figure 1. Regression analysis of the peak load. The average maximum peak load during the first session on the $\mathrm{x}$-axis versus the average maximum peak load during the last session on the $y$-axis. The diagonal dashed line is the $\mathrm{x}=\mathrm{y}$ line. Every point above this line indicates improvements in maximum peak load during rehabilitation. This figure illustrates that 14 patients in the control group deteriorated during rehabilitation, versus 2 patients in the intervention group. It can also be found that most of the patients improved during rehabilitation. Moreover, the patients with real-time biofeedback showed higher improvements compared to the control group. This effect is especially found in the patients that started with a low peak load $(<62 \% \mathrm{BW}$, represented by the vertical dashed line).

\section{\begin{tabular}{|l|l|l}
\hline Mobility test & Control group & Intervention group
\end{tabular}}

\begin{tabular}{|l|l|l|l|l|l|l|}
\hline & Start & End & P & Start & End & P \\
\hline EMS & $15.0 \pm 2.91$ & $17.6 \pm 1.87$ & $<0.0001$ & $14.8 \pm 3.88$ & $17.5 \pm 1.93$ & $<0.0001$ \\
\hline FAC & $3.37 \pm 0.79$ & $4.03 \pm 0.59$ & $<0.0001$ & $3.32 \pm 0.58$ & $3.96 \pm 0.35$ & $<0.0001$ \\
\hline VAS & $4.09 \pm 2.07$ & $2.10 \pm 1.87$ & $<0.0001$ & $4.72 \pm 1.69$ & $2.70 \pm 1.98$ & $<0.0001$ \\
\hline
\end{tabular}

Table 3. Mobility scores at the start and end of the rehabilitation. Significant improvements were found in all mobility tests. 


\section{Discussion}

Hip fractures in elderly have a high morbidity and high mortality [2,3]. High quality rehabilitation programs are necessary to improve fracture healing outcome and quality of life. Previously, it was shown that biofeedback systems are able to monitor the rehabilitation progress and measure important gait parameters [14]. This raised the question if biofeedback systems could be used to improve rehabilitation by real-time biofeedback on important gait parameters. The current randomised controlled trial showed significant improvements in weight bearing if real-time biofeedback was given in the ambulatory, clinical setting. These results pave the way to implement biofeedback systems in innovative rehabilitation programs and develop worldwide guidelines for rehabilitation after hip fractures in elderly.

Similar to findings in previous studies, it was shown in current study that SensiStep is able to gain insight in gait patterns and monitor the rehabilitation progress. It was found that the gait parameters maximum peak load and step duration significantly improved during rehabilitation. Moreover, the current study has shown an increased effect in improvements if real-time biofeedback was given by SensiStep, which demonstrated the positive effect of using biofeedback systems in rehabilitation programs. Interestingly, individual patients responded different to real-time biofeedback, as some patients have more advantage from biofeedback than other patients. A detailed look at patients' characteristics learned that particularly patients who start with a low peak load showed positive effects of biofeedback. This suggests that biofeedback has mainly beneficial effects on patients that start in (the previously defined) groups 3 and 4 with their rehabilitation [14]. Subsequently, differentiated and tailor-made rehabilitation programs should be developed were especially patients in group 3 and 4 receive biofeedback from SensiStep. In contrast, patients in group 1 and 2 could focus on other aspects than weight bearing, for example the step duration.

The effect of biofeedback on the step duration, however, was limited in the current study. Also, previous studies were not consistent about the effect of biofeedback on other parameters than maximum peak load $[11,19]$. This was attributed to the method of biofeedback, as this was only given on the maximum peak load instead of other gait parameters as well [13]. The option to provide biofeedback on step duration should therefore be considered in the ongoing development of biofeedback devices such as SensiStep. This could also lead to a more differentiated approach, that offers the 
opportunity for innovative and tailor-made rehabilitation programs to focus on either improving weight bearing (i.e. group 3 and 4), or step duration (i.e. group 2 and 4). Moreover, patients in group 1 show good gait patterns and must aim to continue their rehabilitation at home, while patients in group 4 need more training sessions under direct supervision of the healthcare professional. Another method to further improve the gait is to increase the exposure of participants to feedback. In the current study protocol, biofeedback was provided daily in a 30-meter walk, up to a maximum of five days per week. Continuous unsupervised feedback might improve the progress even further.

Another important aspect was to evaluate if patients actually rehabilitate faster if the gait pattern was improved by using SensiStep. The hypothesis was that the length of stay in the rehabilitation clinics would be significantly shorter in the intervention group compared to the control group. However, no significant differences between both groups were found. This could be explained by the fact that other aspects than the ability to walk determine the discharge date as well, such as access to home care, the presence of a healthy partner, and the current home environment, such as stairs, split level etcetera [16]. With these arguments in mind, the rehabilitation progress in current study was not related to the discharge date, but it was investigated if patients in the intervention group moved faster towards the previously described objective endpoint of rehabilitation [14]. As indicated by our results, patients did move significantly faster towards the optimal maximum peak load. In contrast, patients did not move faster towards the optimal step duration. It was found that an improvement of both parameters is necessary to move faster towards the objective endpoint of rehabilitation.

One of the strengths of current study are the similar findings measured by SensiStep and the validated mobility tests EMS and FAC. All results showed significant improvements between the start and end of rehabilitation in both groups. Also, improvements made during the rehabilitation were indicated by lower VAS scores over time. Remarkable is that only the gait parameter maximum peak load showed significant higher improvements in the intervention group compared to the control group. This could indicate that maximum peak load is the most sensitive parameter to analyse the rehabilitation progress. Also, this parameter is more objectively measured by SensiStep compared to the mobility tests, which are subjective and operator dependent [20]. 
A second strength is the study design and population in current study. Patients are randomised into two groups and included in eleven different geriatric rehabilitation centers in two European countries. Therefore, the results of current study can be translated to elderly patients rehabilitating in other rehabilitation clinics and countries as well. The differences in the treating physician, institutional protocol, cultural or national differences appear not to contribute to the beneficial effect of real-time biofeedback on rehabilitation after hip fractures in elderly.

\section{Conclusion}

Biofeedback systems such as SensiStep are helpful to improve therapy compliance after hip fractures in elderly. Significant improvements in maximum peak load were found by giving real-time biofeedback. Other feedback mechanisms need to be developed in the ongoing development of SensiStep to provide feedback about other important gait parameters as well, such as step duration. Moreover, the current results should be used to develop new tailor-made rehabilitation programs for elderly patients rehabilitating from a hip fracture. 


\section{References}

[1] C. M. M. Peeters, E. Visser, C. L. P. Van De Ree, T. Gosens, B. L. Den Oudsten, and J. De Vries, "Quality of life after hip fracture in the elderly: A systematic literature review," Injury, vol. 47, no. 7, pp. 1369-1382, 2016.

[2] S. E. Roberts and M. J. Goldacre, "Time trends and demography of mortality after fractured neck of femur in an English population, 1968-98: database study,” BMJ, vol. 327, no. October, pp. 1-5, 2003.

[3] X. L. Griffin, N. Parsons, J. Achten, M. Fernandez, and M. L. Costa, "Recovery of health-related quality of life in a United Kingdom hip fracture population: The warwick hip trauma evaluation - A prospective cohort study," Bone Jt. J., vol. 97-B, no. 3, pp. 372-382, 2015.

[4] M. Bhandari, P. J. Devereaux, T. A. Einhorn, L. Thabane, E. H. Schemitsch, K. J. Koval, F. Frihagen, R. W. Poolman, K. Tetsworth, E. Guerra-Farfán, K. Madden, S. Sprague, and G. Guyatt, "Hip fracture evaluation with alternatives of total hip arthroplasty versus hemiarthroplasty (HEALTH): Protocol for a multicentre randomised trial," BMJ Open, vol. 5, no. 2, pp. 1-12, 2015.

[5] M. J. Parker, "Sliding hip screw versus intramedullary nail for trochanteric hip fractures; a randomised trial of 1000 patients with presentation of results related to fracture stability," Injury, vol. 48, no. 12, pp. 2762-2767, 2017.

[6] J. Klein-Nulend, R. G. Bacabac, and M. G. Mullender, "Mechanobiology of bone tissue," Pathol. Biol., vol. 53, no. 10 , pp. $576-580,2005$.

[7] F. K. Ruiz, M. C. Fu, D. D. Bohl, J. W. Hustedt, M. R. Baumgaertner, M. P. Leslie, and J. N. Grauer, "Patient Compliance With Postoperative Lower Extremity Touch-down Weight-bearing Orders at a Level I Academic Trauma Center," Orthopedics, vol. 37, no. 6, pp. e552-e556, 2014.

[8] A. Vasarhelyi, T. Baumert, C. Fritsch, W. Hopfenmüller, G. Gradl, and T. Mittlmeier, "Partial weight bearing after surgery for fractures of the lower extremity - is it achievable?," Gait Posture, vol. 23, no. 1, pp. 99-105, 2006.

[9] J. W. Hustedt, D. J. Blizzard, M. R. Baumgaertner, M. P. Leslie, and J. N. Grauer, "Is it possible to train patients to limit weight bearing on a lower extremity?," Orthopedics, vol. 35, no. 1, pp. e31-7, 2012.

[10] B. J. Braun, N. T. Veith, M. Rollmann, M. Orth, T. Fritz, S. C. Herath, J. H. Holstein, and T. Pohlemann, "Weight-bearing recommendations after operative fracture treatment - fact or fiction? Gait results with and feasibility of a dynamic, continuous pedobarography insole," Int. Orthop., pp. 1-6, 2017.

[11] M. Raaben, H. R. Holtslag, L. P. H. Leenen, R. Augustine, and T. J. Blokhuis, "Real-time visual biofeedback during weight bearing improves therapy compliance in patients following lower extremity fractures," Gait Posture, vol. 59, no. October 2017, pp. 206-210, 2018.

[12] H. L. P. Hurkmans, J. B. J. Bussmann, E. Benda, J. a N. Verhaar, and H. J. Stam, "Techniques for measuring weight bearing during standing and walking," Clin. Biomech., vol. 18, no. 7, pp. 576-589, 2003.

[13] M. Raaben, H. C. Vogely, and T. J. Blokhuis, "Real-time visual biofeedback to improve therapy compliance after total hip arthroplasty: A pilot randomized controlled trial,” Gait Posture, vol. 61, no. January, pp. 306-310, 2018.

[14] M. Raaben, S. M. S. Redzwan, R. Augustine, and T. J. Blokhuis, "Innovative Measurement Of Rehabilitation Progress In Elderly With A Hip Fracture : A New Endpoint,” 2018 IEEE Conf. Antenna Meas. Appl., pp. 1-4, 2018. 
[15] M. W. Rosanna Chau, S. P. Chan, Y. W. Wong, and M. Y. P. Lau, "Reliability and validity of the Modified Functional Ambulation Classification in patients with hip fracture," Hong Kong Physiother. J., vol. 31, no. 1, pp. 41-44, 2013.

[16] Verenso, “Triage Instrument Geriatrische Revalidatiezorg,” 2012.

[17] M. Raaben, H. Holtslag, R. Augustine, R. van Merkerk, B. Koopman, and T. Blokhuis, "Technical Aspects and Validation of a New Biofeedback System for Measuring Lower Limb Loading in the Dynamic Situation,” Sensors, vol. 17 , no. 3 , p. $658,2017$.

[18] M. Raaben, S. Redzwan, R. Augustine, and T. J. Blokhuis, "COMplex Fracture Orthopedic Rehabilitation (COMFORT) - Real-time visual biofeedback on weight bearing versus standard training methods in the treatment of proximal femur fractures in the elderly: Study protocol for a multicenter randomized controlled tria," Trials, vol. 19, no. 1, pp. 1-7, 2018.

[19] A. Bakker, T. J. Blokhuis, M. D. M. E. Meeks, H. J. Hermens, and H. R. Holtslag, "Dynamic weight loading in older people with hip fracture.," J. Rehabil. Med., pp. 1-4, 2014.

[20] N. A. De Morton, J. Nolan, M. O’Brien, S. Thomas, A. Govier, K. Sherwell, B. Harris, and N. Markham, “A head-to-head comparison of the de Morton Mobility Index (DEMMI) and Elderly Mobility Scale (EMS) in an older acute medical population,” Disabil. Rehabil., vol. 37, no. 20, pp. 1881-1887, 2015. 
CHAPTER

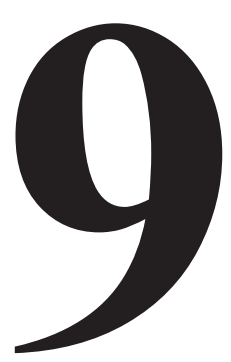


General discussion and summary 


\section{Introduction}

The development of complex and innovative medical technologies is increasing over the last decade. These technologies are used in all medical areas, such as prevention, diagnostics and therapies. Although the developments in all areas are interesting and extremely valuable, this thesis focused on the development of innovative and state-of-the art biofeedback systems designed for rehabilitation programs after lower extremity fractures. Biofeedback systems have a long history, as the first system was already described in 1974 by Endicott et al. This system consisted of two audible tones, a low frequency tone if the patient had not exceeded the minimum load and a high-frequency tone if the patient had exceeded the maximum load [1]. Ever since, great improvements have been made to biofeedback systems.

Currently, biofeedback systems are widely used in rehabilitation programs after various diseases, such as stroke, diabetes, amputations and lower extremity fractures. Patients rehabilitating after lower extremity fractures often have strict prescribed weight bearing regimes, varying between no weight bearing, touch-down weight bearing, partial weight bearing and full weight bearing. Remarkable is the difference and lack of consensus for the prescribed weight bearing regime, as this is mainly experience based instead of evidence based. Moreover, several studies have shown that therapy compliance was low if standard training methods were used [2-5]. Multiple studies have shown the potential of biofeedback systems to improve therapy compliance after lower extremity fractures [6-8]. However, none of the biofeedback systems used in these studies fulfilled the high requirements demanded for clinical use [9].

The first aim of this thesis was therefore to develop an innovative, ambulatory biofeedback system able to provide real-time, visual biofeedback to patients in the clinical setting. Then, this system was used to develop innovative rehabilitation programs after lower extremity fractures, with a special focus on hip fractures in elderly.

\section{The development of SensiStep}

Researchers have a long interest in the development and use of biofeedback systems to improve gait in various diseases, such as stroke, diabetes, amputations and lower extremity fractures. Hurkmans et al. have provided an overview of the development of biofeedback systems over the last decades [9]. The authors came to the conclusion that all devices are 
promising, but have limitations, such as restricted to laboratory use, inaccurate, use of cables and lack of availability. Biofeedback systems must meet high requirements, including a high level of mobility, limited cabling, correct sensor placement, low power consumption and low costs [10]. The first aim of this thesis was to develop a new biofeedback system that satisfied these high requirements. This resulted in the development of SensiStep (chapter 3). The state-of-the art sensor technologies, based on the Hall-effect, resulted in accurate and reliable load measurements in both static as dynamic loading. These results were highly reproducible, irrespective of sensor placement and without the daily need for sensor calibration. The use of Bluetooth Low Energy as communication method ensured that the system is wireless and has low energy consumption. Finally, the system has CE marking and is relatively affordable compared to other commercially available biofeedback devices. These properties ensure that SensiStep can safely and easily be used in the ambulatory, clinical setting in order to improve weight bearing.

\section{The importance of weight bearing}

Julius Wolff has described the importance of mechanical load in bone remodelling [11]. This also applies for bone healing after fractures. Mechanical load induces micromovement at the fracture site leading to a process known as mechanotransduction in bone [12]. This suggested that correct and early weight bearing has beneficial effects on fracture healing after lower extremity fractures. This hypothesis was studied in chapter 2, were the importance of early weight bearing on fracture healing after tibial shaft fractures was described. Several variables were found to have a significant effect on fracture healing outcome, including infection requiring procedure (OR: 5.23), fracture type (OR: 2.19), fasciotomy (OR: 3.39) and open fracture (OR: 1.84). Moreover, the timing of initial weight bearing also significantly affected fracture healing outcome, accounting for an odds ratio of 1.13 per week delay. Since the timing of initial weight bearing is under the direct influence of the treating physician, this is certainly an interesting variable to improve fracture healing outcome. It can be concluded from chapter 2 that early weight bearing is preferable and has beneficial effects on fracture healing outcome after lower extremity fractures.

Although the beneficial effects of early weight bearing on fracture healing are known, it is remarkable that there is still no consensus about the correct weight bearing regime after lower extremity fractures. There is an absence of worldwide guidelines and the prescribed training regime is mainly experienced based and differs per treating physician. Moreover, 
previous studies have shown that therapy compliance is low, both in partial weight bearing as in full weight bearing [2-5]. This was also confirmed by the results described in chapter 4, where it was found that patients with touch-down weight bearing structurally overload their lower extremity $(16.7 \pm 9.77 \mathrm{~kg})$, with potentially dangerous maximum peak loads up to $42 \mathrm{~kg}$. This could lead to mechanical construct failure or fixation failure at the fracture site. In contrast, patients with full weight bearing structurally underload the fractured extremity to only $50.9 \pm 7.51 \%$ of their bodyweight. As early weight bearing has significant positive effects on fracture healing outcome, as described in chapter $\mathbf{2}$, underloading the fracture site could lead to delayed fracture healing or even non-union.

The second aim of this thesis was therefore to improve therapy compliance after lower extremity fractures. In chapter $\mathbf{4}$ it was found that both touch-down and full weight bearing significantly improved if patients received real-time, visual biofeedback from SensiStep. This could lead to better fracture healing outcome. Besides beneficial effects on fracture healing, early weight bearing also leads to improved coordination and muscle mass. This could reduce the risk of falls and thereby new injuries or refractures of the lower extremities. The use of SensiStep was also investigated in elderly patients following a total hip arthroplasty (THA). THA is a common procedure in the treatment of coxarthrosis and patients are usually prescribed full weight bearing to train gait and improve general coordination and muscle mass. However, similar to the limited therapy compliance found after lower extremity fractures, it was described in chapter $\mathbf{5}$ that elderly patients were unable to implement full weight bearing after THA if conventional training methods were used. Especially in the early postoperative phase, significant improvements were found in maximum peak load if real-time, visual biofeedback was given by SensiStep. Other parameters such as loading rate and cadence were investigated as well, but did not show significant improvements if biofeedback was provided. It can be concluded from chapters 4 and 5 that (1) real-time, visual biofeedback about weight bearing leads to higher maximum peak loads, (2) but has limited effects on other gait parameters. Furthermore, (3) biofeedback systems such as SensiStep can be used and have a positive effect on gait in the frail, elderly population. These findings suggested that the system is useful in this specific population, as elderly understand the feedback mechanism and are able to adapt the weight bearing to the optimal level. 


\section{SensiStep after hip fractures in elderly}

This raised the question whether SensiStep could be used to improve rehabilitation after hip fractures in elderly, as this is a major health concern that commonly occurs after low energy trauma in the female elderly (chapter 1). A meticulous surgical fixation is important to allow early weight bearing. Large multicenter clinical trials have investigated the optimal surgical fixation techniques. In contrast, there is an absence of high quality studies that investigate the optimal rehabilitation program. In addition, the standard training methods have a low quality, mainly due to the unavailability of the necessary rehabilitation tools.

The COMplex Fracture Orthopeadic Rehabilitation (COMFORT) project, as described in chapter 6-8, focused on the third aim of this thesis: the development of innovative rehabilitation programs after hip fractures in elderly. The first step in the COMFORT project was to determine the normal gait patterns and rehabilitation progress in this population, as described in chapter 6. Multiple important gait parameters were previous described in literature and investigated in the COMFORT project, such as the maximum peak load, step duration, heel stance time, impulse and loading rate [13]. The results described in this thesis showed that especially the gait parameters maximum peak load and step duration provided a detailed insight in the rehabilitation progress. It was suggested in chapter 6 that our homologous population of elderly patients could be differentiated into four subpopulations, based on the maximum peak load and step duration measured by SensiStep in the first training session. Moreover, an objective endpoint of rehabilitation was defined by a combination of these two parameters, which could replace the currently used subjective and operator dependent mobility scores. The results described in chapter 6 could be used in the development of new rehabilitation programs that focus on training the gait parameters maximum peak load and step duration in order to improve fracture healing outcome.

Before these rehabilitation programs can be implemented, it is important to evaluate if the maximum peak load and step duration could be improved by real-time biofeedback in the frail and elderly population. Therefore, the second step of the COMFORT project was to use SensiStep as innovative biofeedback device and determine the effect of real-time biofeedback, as described in chapter 7-8. First, Chapter 7 described a study protocol to investigate if biofeedback by SensiStep leads to improved gait parameters compared to conventional treatment methods. An international, multicenter, randomised controlled trial 
objectively investigated the effect of biofeedback on rehabilitation progress. Primary outcome measures were the gait parameters maximum peak load and step duration in relation to discharge date and the objective endpoint of rehabilitation. The results of this trial were described in chapter 8. Especially significant higher improvements in maximum peak load were found if SensiStep was used compared to standard training methods. The effect of SensiStep on other gait parameters was limited. This was attributed to the fact that SensiStep provided feedback on the maximum peak load only, instead of feedback on the step duration. However, the results described in chapter $\mathbf{8}$ indicated that frail and elderly patients are able to understand the feedback, and moreover, were able to implement the feedback to adapt the weight bearing to the optimal level. This finding emphasized the importance of using biofeedback systems such as SensiStep in future rehabilitation programs after various diseases, including hip fractures in elderly.

\section{Future perspectives}

Early weight bearing is important to improve coordination, muscle mass and bone remodelling after various types of lower extremity fractures. This thesis has shown that SensiStep can be used as rehabilitation tool to improve early weight bearing after lower extremity fractures. It is recommended to implement SensiStep in future rehabilitation programs to optimize treatment and fracture healing outcome.

The results described in this thesis should be used to develop tailor-made rehabilitation programs for elderly patients following a hip fracture. The patients should be divided into four subpopulations, based on the first training session with SensiStep. If a patient is assigned to a specific subpopulation, the treating healthcare professional could focus on specific needs for that subpopulation, for example improving maximum peak load or step duration. Also, the frequency of training sessions could be adapted to the designated subpopulation. A lower training frequency leads to lower economic costs, while a higher frequency hypothetically leads to faster fracture healing and return to society. Although this thesis described the development, validation and positive effects of an innovative rehabilitation tool, the medical and/or economic effect of tailor-made rehabilitation programs should be kept in mind and investigated in future research.

In addition, improvements in rehabilitation tools are still possible and should also be considered in the ongoing development of biofeedback systems. Specific recommendations for the further development of SensiStep are improvements in the comfort of the sandals 
and the development of parallel feedback mechanisms. Comfort of the sandals is essential to increase the usability of the system. The potential of developing parallel feedback mechanisms is high, as the studies in this thesis have shown that maximum peak load significantly improved by using SensiStep, but in contrast, other gait parameters showed no differences compared to care as usual. This was attributed to the fact that SensiStep only provided feedback about maximum peak load instead of other parameters as well, such as the important gait parameter step duration. Parallel feedback mechanisms about the step duration should be included in SensiStep to improve this parameter as well. This could be achieved by various mechanisms, divided into visual and/or auditory mechanisms. Visual feedback about the step duration could be achieved by adjustments to the green target bar on the tablet. Auditory feedback about the step duration could be achieved by a built-in metronome, with a particular tone on the desired and pre-set step frequency. Other mechanisms such as virtual or augmented reality could also be considered. Finally, feedback mechanisms about other gait parameters could be integrated, such as loading rate and targeted number of steps per day.

In conclusion, it is recommended to use biofeedback system in rehabilitation after lower extremity fractures to improve fracture healing outcome. The results described in this thesis could be used as guideline to develop worldwide rehabilitation programs for specific populations. Simultaneously, it is necessary to further develop the existing biofeedback systems to increase the comfort and use of such systems in the future. 


\section{References}

[1] D. Endicott, R. Roemer, S. Brooks, H. Meisel, Leg load warning system for the orthopaedically handicapped, Med. Biol. Eng. 12 (1974) 318-321.

[2] F.K. Ruiz, M.C. Fu, D.D. Bohl, J.W. Hustedt, M.R. Baumgaertner, M.P. Leslie, J.N. Grauer, Patient Compliance With Postoperative Lower Extremity Touch-down Weight-bearing Orders at a Level I Academic Trauma Center, Orthopedics. 37 (2014) e552-e556. doi:10.3928/01477447-20140528-55.

[3] A. Vasarhelyi, T. Baumert, C. Fritsch, W. Hopfenmüller, G. Gradl, T. Mittlmeier, Partial weight bearing after surgery for fractures of the lower extremity - is it achievable?, Gait Posture. 23 (2006) 99-105. doi:10.1016/j.gaitpost.2004.12.005.

[4] J.W. Hustedt, D.J. Blizzard, M.R. Baumgaertner, M.P. Leslie, J.N. Grauer, Is it possible to train patients to limit weight bearing on a lower extremity?, Orthopedics. 35 (2012) e31-7. doi:10.3928/01477447-20111122-14.

[5] B.J. Braun, N.T. Veith, M. Rollmann, M. Orth, T. Fritz, S.C. Herath, J.H. Holstein, T. Pohlemann, Weightbearing recommendations after operative fracture treatment—-fact or fiction? Gait results with and feasibility of a dynamic, continuous pedobarography insole, Int. Orthop. (2017) 1-6. doi:10.1007/s00264-017-3481-7.

[6] Z. Pataky, D. De León Rodriguez, A. Golay, M. Assal, J.P. Assal, C.A. Hauert, Biofeedback Training for Partial Weight Bearing in Patients After Total Hip Arthroplasty, Arch. Phys. Med. Rehabil. 90 (2009) 1435-1438. doi:10.1016/j.apmr.2009.02.011.

[7] M.C. Fu, L. DeLuke, R. a Buerba, R.E. Fan, Y.J. Zheng, M.P. Leslie, M.R. Baumgaertner, J.N. Grauer, Haptic biofeedback for improving compliance with lower-extremity partial weight bearing., Orthopedics. 37 (2014) e993-8. doi:10.3928/01477447-20141023-56.

[8] E. Hershko, C. Tauber, E. Carmeli, Biofeedback Versus Physiotherapy in Patients With Partial Weight-Bearing, Am. J. Orthop. 37 (2008) 92-96.

[9] H.L.P. Hurkmans, J.B.J. Bussmann, E. Benda, J. a N. Verhaar, H.J. Stam, Techniques for measuring weight bearing during standing and walking, Clin. Biomech. 18 (2003) 576-589. doi:10.1016/S0268-0033(03)00116-5.

[10] A.H. Abdul Razak, A. Zayegh, R.K. Begg, Y. Wahab, Foot plantar pressure measurement system: A review, Sensors. 12 (2012) 9884-9912. doi:10.3390/s120709884.

[11] J. Wolff, Das Gesetz der Transformation der Knochen, 1892.

[12] J. Klein-Nulend, R.G. Bacabac, M.G. Mullender, Mechanobiology of bone tissue, Pathol. Biol. 53 (2005) $576-$ 580. doi:10.1016/j.patbio.2004.12.005.

[13] A. Bakker, T.J. Blokhuis, M.D.M.E. Meeks, H.J. Hermens, H.R. Holtslag, Dynamic weight loading in older people with hip fracture., J. Rehabil. Med. (2014) 1-4. doi:10.2340/16501977-1793. 
CHAPTER

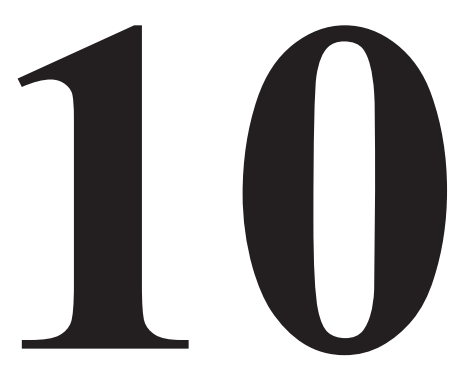


Nederlandse samenvatting en toekomstperspectieven (Dutch summary) 


\section{Introductie}

De ontwikkeling van complexe en innovatieve medische technologieën heeft een enorme groei doorgemaakt in het afgelopen decennium. Deze technologieën worden ingezet voor allerlei medische doeleinden, zoals preventie, diagnostiek en behandeling. Dit proefschrift richt zich op de ontwikkeling van technologische hulpmiddelen voor de behandeling en met name de revalidatie na fracturen van de onderste extremiteiten (de onderste ledematen). Het eerste doel van dit proefschrift was het ontwikkelen van een innovatief en modern biofeedback systeem. Biofeedback systemen hebben een lange geschiedenis in wetenschappelijk onderzoek. Het eerste systeem werd al in 1974 beschreven door Endicott en collega's. Dit systeem bestond uit twee auditieve tonen, (1) een laag frequente toon als een patiënt niet de minimale doelbelasting haalde en (2) een hoog frequente toon als de maximale doelbelasting werd overschreden. Sindsdien zijn deze biofeedback systemen verder ontwikkeld en zijn er grote verbeteringen doorgevoerd.

Op dit moment worden biofeedback systemen gebruikt in revalidatieprogramma's voor uiteenlopende ziektebeelden, zoals na een beroerte, diabetes, amputaties en fracturen van de onderste extremiteiten. Het gebruik van biofeedback systemen kan met name verbetert worden in revalidatieprogramma's na fracturen van de onderste extremiteiten. Deze patiënten krijgen vaak een strikte doelbelasting voorgeschreven, variërend tussen niet belasten, aantippend belasten, gedeeltelijk belasten en volledig belasten. Hierbij valt direct op dat er een groot verschil is in voorgeschreven doelbelasting en dat er ook geen consensus bestaat tussen verschillende artsen over de correcte doelbelasting. De voorgeschreven doelbelasting is namelijk meestal gebaseerd op de ervaring van een individuele arts, in plaats van bewijs verkregen door wetenschappelijk onderzoek. Daarnaast hebben diverse studies aangetoond dat de therapietrouw van de patiënten erg laag is. Er is dus nog veel ruimte voor verbetering in de belasting na fracturen van de onderste extremiteiten. Eerdere studies hebben de potentie van biofeedback systemen tijdens de revalidatie al aangetoond, waarbij een significante verbetering in de therapietrouw werd gevonden. Echter, geen van de biofeedback systemen die gebruikt werden voor deze studies voldeden aan de hoge eisen die nodig zijn voor gebruik in de kliniek.

Het eerste doel van dit proefschrift was daarom het ontwikkelen van een innovatief en ambulant biofeedback systeem dat in staat is om real-time, visueel biofeedback te geven 
aan patiënten in de kliniek. Daarna werd dit systeem gebruikt om nieuwe revalidatieprogramma's te ontwikkelen voor patiënten die revalideren na fracturen van de onderste extremiteiten, waarbij de focus lag op heupfracturen bij ouderen.

\section{De ontwikkeling van SensiStep}

Onderzoekers zijn al een lange tijd geïnteresseerd in de ontwikkeling en het gebruik van biofeedback systemen ter verbetering van het looppatroon bij verschillende ziektebeelden, zoals een beroerte, diabetes, amputaties en fracturen van de onderste extremiteiten. Hurkmans en collega's hebben een overzicht gegeven met de ontwikkeling van biofeedback systemen tijdens de afgelopen decennia. Ze kwamen daarbij tot de conclusie dat alle biofeedback systemen veelbelovend zijn, maar dat ze ook allemaal hun tekortkomingen hebben. Hierbij valt te denken aan de beperking tot gebruik in een laboratorium, onnauwkeurigheid, het gebruik van kabels, maar ook het gebrek aan beschikbaarheid. Biofeedback systemen moeten voldoen aan hoge eisen, zoals goede mobiliteit, beperkt gebruik van kabels, een goede positie van de sensor, laag energiegebruik en lage prijs. Het eerste doel van dit proefschrift was dan ook het ontwikkelen van een biofeedback systeem dat voldeed aan deze hoge eisen. Dit resulteerde in de ontwikkeling van SensiStep, zoals beschreven in hoofdstuk 3. De moderne sensor technologie, gebaseerd op het Hall-effect, resulteerde in nauwkeurige, betrouwbare en reproduceerbare metingen van de belasting. Het gebruik van Bluetooth Low Energy als communicatie methode zorgt ervoor dat het systeem draadloos is en weinig energie verbruikt. Daarnaast heeft het systeem een CEmarkering en is het relatief goedkoop in vergelijking met andere commercieel beschikbare biofeedback systemen. Deze eigenschappen zorgen ervoor dat SensiStep veilig en gemakkelijk gebruikt kan worden in de ambulante, klinische omgeving om de belasting te verbeteren.

\section{Het belang van belasten}

Julius Wolff beschreef al in de $19^{\mathrm{e}}$ eeuw het belang van belasten op de botvorming. Later is ontdekt dat belasten ook belangrijk is voor botgenezing na fracturen. Belasting zorgt namelijk voor een kleine beweging in de fractuur, dat een proces in werking zet genaamd 'mechanotransductie'. Dit suggereert dat snel en goed belasten na fracturen van de onderste extremiteiten gunstig is voor de fractuurgenezing. Deze hypothese werd onderzocht in hoofdstuk 2 van dit proefschrift. In dit hoofdstuk werd het belang van vroeg belasten op de fractuurgenezing beschreven. Verschillende onderwerpen hadden een significant effect op 
de uitkomst van de fractuurgenezing, zoals een infectie (OR: 5.23), fractuurtype (OR: 2.19), fasciotomie (OR: 3.39) en een open fractuur (OR: 1.84). Bovendien had ook de timing van belasten een significant effect op de fractuurgenezing, met een odds ratio van 1.13 per week vertraging. Aangezien de timing van belasten onder de directe invloed is van de behandeld arts, is dit een interessante variabele die gebruikt kan worden om de fractuurgenezing verder te verbeteren. Het kan uit hoofdstuk 2 geconcludeerd worden dat vroeg belasten wordt aanbevolen vanwege de gunstige effecten op de uitkomst van de fractuurgenezing.

Op basis van deze gegevens is het opmerkelijk dat er tussen de behandelend artsen nog steeds geen consensus bestaat over de correcte mate van belasten na fracturen van de onderste extremiteiten. Er is geen wereldwijde richtlijn beschikbaar en de voorgeschreven belasting is grotendeels gebaseerd op de ervaring van de arts in plaats van wetenschappelijk onderzoek. Bovendien hebben voorgaande studies laten zien dat de therapietrouw van patiënten erg laag is, zowel bij partieel belasten als bij volledig belasten. Deze resultaten worden ook bevestigd in hoofdstuk 4 van dit proefschrift, waar werd aangetoond dat patiënten met voorgeschreven 'aantippend belasten' hun onderste extremiteit structureel overbelasten $(16.7 \pm 9.77 \mathrm{~kg})$, met gevaarlijke uitschieters tot $42 \mathrm{~kg}$. Dit kan leiden tot het breken van het fixatie materiaal of zelfs het opnieuw breken van de fractuur. Aan de andere kant belasten patiënten met voorgeschreven 'volledig belasten' hun onderste extremiteit tot maar $50.9 \pm 7.51 \%$ van hun lichaamsgewicht. Aangezien in hoofdstuk 2 werd aangetoond dat vroeg en goed belasten een significant positief effect heeft op de fractuurgenezing, kan te weinig belasten van de fractuur leiden tot vertraagde of zelfs geen fractuurgenezing.

Het tweede doel van dit proefschrift was daarom de therapietrouw verbeteren na fracturen van de onderste extremiteiten. In hoofdstuk 4 werd beschreven dat zowel patiënten met aantippend belasten als volledig belasten significant verbeterden als ze real-time, visuele biofeedback kregen van SensiStep. Naast de gunstige effecten die beter belasten heeft op de fractuurgenezing, zorgt vroeg belasten ook voor een verbetering in de coördinatie en spierkracht. Hierdoor is er minder risico op vallen en daarbij nieuwe fracturen van de onderste extremiteiten. Daarom werd het gebruik van SensiStep ook onderzocht bij oudere patiënten met een totale heupprothese als behandeling voor heupartrose. Een totale heupprothese is een veelgebruikte procedure in de behandeling van heupartrose en patiënten mogen meestal direct volledig belasten om hun looppatroon te trainen en hun coördinatie en spierkracht te verbeteren. In hoofdstuk 5 werd echter aangetoond dat de 
therapietrouw erg laag was, net zoals bij patiënten met fracturen van de onderste extremiteiten. De patiënten waren niet in staat om hun onderste extremiteiten volledig te belasten als de standaard training methodes werden gebruikt. Met name in de vroege fase na operatie werd een significante verbetering in maximale piekbelasting gevonden met realtime, visuele biofeedback door SensiStep. Andere parameters werden ook onderzocht, maar lieten geen significante verbetering zien na het geven van biofeedback. Uit hoofdstuk $\mathbf{4}$ en 5 kunnen drie conclusies worden getrokken, namelijk dat (1) real-time, visuele biofeedback over de belasting zorgt voor een hogere maximale piekbelasting, (2) maar een minimaal effect heeft op overige parameters. Verder (3) hebben biofeedback systemen zoals SensiStep een positief effect bij de specifieke populatie van kwetsbare ouderen. Deze bevinding suggereert dat SensiStep bruikbaar is in deze populatie, mede doordat kwetsbare ouderen het feedback mechanisme begrijpen èn ze de belasting kunnen aanpassen naar het optimale niveau.

\section{SensiStep na heupfracturen bij ouderen}

Dit leidt naar de vraag of SensiStep ook gebruikt kan worden voor het verbeteren van de revalidatie na heupfracturen bij ouderen. Dit ziektebeeld heeft een grote impact heeft op veel kwetsbare ouderen. Heupfracturen komen met name voor bij oudere vrouwen na een val vanuit stand (hoofdstuk 1). Zorgvuldige operatietechnieken zijn nodig om patiënten in staat te stellen om de fractuur vroeg te belasten. Daarom hebben diverse grote klinische studies onderzocht wat de optimale operatietechnieken zijn. Er is echter maar beperkt onderzoek gedaan naar de optimale revalidatieprogramma's. Bovendien zijn de standaard training methodes van lage kwaliteit, mede ook doordat de noodzakelijke revalidatie hulpmiddelen niet beschikbaar zijn.

Het COMplex Fracture Orthopaedic Rehabilitation (COMFORT) project, zoals beschreven in hoofdstuk 6-8, focust op het derde doel van dit proefschrift: het ontwikkelen van innovatieve revalidatieprogramma's na heupfracturen bij ouderen. De eerste stap in het COMFORT project was om te bepalen wat het normale looppatroon en revalidatieverloop in deze populatie was, zoals beschreven in hoofdstuk 6. In dit hoofdstuk is onderzoek gedaan naar meerdere belangrijke parameters die in eerdere literatuur werden beschreven, zoals de maximale piekbelasting, stapduur, hielstand tijd, impuls en loading rate. De resultaten van dit proefschrift hebben aangetoond dat met name de parameters maximale piekbelasting en stapduur een gedetailleerd inzicht gaven in het normale looppatroon en 
revalidatieverloop na een heupfractuur bij ouderen. Het werd gesuggereerd in hoofdstuk 6 dat er vier subpopulaties gevormd konden worden op basis van de maximale piekbelasting en de stapduur gemeten door SensiStep tijdens de eerste trainingssessie. Bovendien kon door een combinatie van deze twee parameters het objectief eindpunt van revalidatie bepaald worden, dat het momenteel gebruikte subjectieve eindpunt kan vervangen. De resultaten zoals beschreven in hoofdstuk 6 kunnen gebruikt worden voor de ontwikkeling van nieuwe revalidatieprogramma's, die focussen op het trainen van de parameters maximale piekbelasting en stapduur.

Voor de ontwikkeling van deze revalidatieprogramma's is het echter belangrijk om te onderzoeken of de maximale piekbelasting en de stapduur verbetert kunnen worden door het geven van real-time biofeedback aan kwetsbare ouderen. Daarom was de tweede stap in het COMFORT project om SensiStep te gebruiken als innovatief biofeedback systeem om het effect van real-time biofeedback te bepalen bij ouderen met een heupfractuur, zoals beschreven in hoofdstuk 7-8. Allereerst wordt in hoofdstuk 7 een studieprotocol beschreven dat onderzoekt of biofeedback door SensiStep leidt tot een verbetering in de parameters vergeleken met de standaard training methodes. Deze internationale studie onderzocht het effect van biofeedback op het looppatroon en het revalidatieverloop. De primaire uitkomstmaten waren de parameters maximale piekbelasting en stapduur in relatie met de ontslagdatum en het objectieve eindpunt van revalideren. De resultaten van deze studie zijn beschreven in hoofdstuk 8. Met name werd een significante verbetering in de maximale piekbelasting gevonden door het gebruik van SensiStep in vergelijking met de standaard training methodes. Het effect van SensiStep op de andere parameters was minimaal. Dit werd toegeschreven aan het feit dat SensiStep alleen feedback geeft op de maximale piekbelasting en niet op andere parameters, zoals de stapduur. De resultaten in hoofdstuk 8 laten zien dat de kwetsbare ouderen in staat zijn om de feedback te begrijpen èn ook toe te passen om hun belasting te verbeteren naar het optimale niveau. Deze bevinding benadrukt het belang van biofeedback systemen zoals SensiStep in toekomstige revalidatieprogramma's bij verschillende ziektebeelden, inclusief heupfracturen bij ouderen.

\section{Toekomstperspectieven}

Vroeg belasten is belangrijk voor het verbeteren van de coördinatie, spierkracht en bothervorming na verschillende type fracturen van de onderste extremiteiten. Dit 
proefschrift heeft aangetoond dat SensiStep gebruikt kan worden als revalidatie instrument voor het verbeteren van belasten na fracturen van de onderste extremiteiten. Het wordt daarom aanbevolen om SensiStep te implementeren in toekomstige revalidatieprogramma's om de behandeling en fractuurgenezing te optimaliseren.

De beschreven resultaten in dit proefschrift kunnen gebruikt worden om op maat gemaakte revalidatieprogramma's te ontwikkelen voor ouderen met een heupfractuur. Deze patiënten kunnen verdeeld worden in vier subpopulaties gebaseerd op de eerste training met SensiStep. Indien een patiënt is toegewezen aan een bepaalde subpopulatie kan de behandelend fysiotherapeut de focus leggen op de specifieke behoeften behorend bij die subpopulatie, bijvoorbeeld het verbeteren van de maximale piekbelasting of de stapduur. Ook kan er rekening worden gehouden met de frequentie van de trainingssessies. Minder trainingen zal ook leiden tot minder kosten, terwijl meer trainingen kunnen zorgen voor snellere fractuurgenezing en daarbij snellere terugkeer naar de maatschappij. Hoewel dit proefschrift met name de ontwikkeling, validatie en positieve effecten van een innovatief biofeedback systeem heeft beschreven, moet er in toekomstig onderzoek nagedacht worden over de medische en/of economische effecten van op maat gemaakte revalidatieprogramma's.

Daarnaast moet er nagedacht worden om de bestaande revalidatie instrumenten te verbeteren in de voortdurende ontwikkeling van biofeedback systemen. Aanbevelingen voor de ontwikkeling van SensiStep zijn met name verbeteringen in het comfort van de sandalen en het ontwikkelen van parallelle feedback mechanismen. Het verbeteren van het comfort van de sandalen is essentieel om het gebruik van SensiStep te laten toenemen. Het potentieel van parallelle feedback mechanismen is groot, mede aangezien de studies in dit proefschrift hebben aangetoond dat de maximale piekbelasting significant verbetert door biofeedback, maar dat andere parameters geen effect lieten zien in vergelijking met de standaard therapie. Dit werd toegeschreven aan het feit dat SensiStep alleen feedback geeft over de maximale piekbelasting, maar niet over andere parameters zoals de stapduur. Parallelle feedback mechanismen over de stapduur zouden ook ontwikkeld moeten worden in SensiStep. Er zijn verschillende manieren waarop dit bereikt zou kunnen worden, zowel visueel als auditief. Visuele feedback over de stapduur kan bijvoorbeeld gegeven worden door de weergave van de doelbelasting te veranderen. Auditieve feedback over de stapduur kan bereikt worden door een metronoom in SensiStep te bouwen, met een afgespeelde toon 
op de gewenste stapfrequentie. Tot slot moeten ook feedbackmechanismen voor andere parameters geïntegreerd worden, zoals de loading rate en het gewenste aantal stappen per dag.

Concluderend wordt het aanbevolen om biofeedback systemen te gebruiken tijdens de revalidatie na fracturen van de onderste extremiteiten om de fractuurgenezing te verbeteren. De beschreven resultaten in dit proefschrift kunnen als richtlijn gebruikt worden om wereldwijde revalidatieprogramma's te ontwikkelen voor specifieke patiënten. Daarnaast is het noodzakelijk om de bestaande biofeedback systemen verder te ontwikkelen om het comfort en gebruik van deze systemen te vergroten. 
ADDENDUM

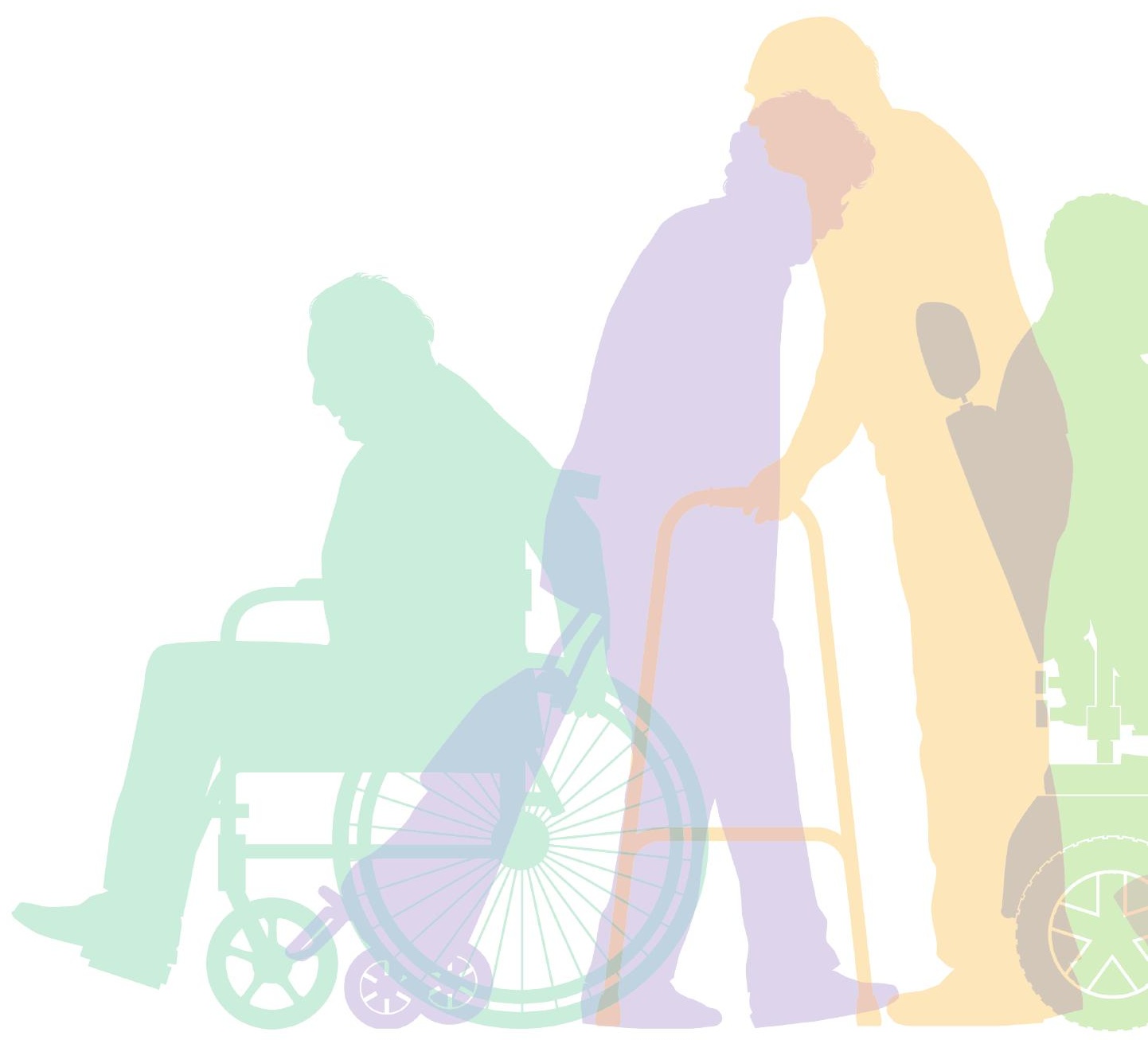


Valorisation

List of publications

International abstracts and poster sessions

Acknowledgements

Curriculum Vitae 


\section{Valorisation}

\section{Relevance}

Healthcare is extremely expensive and innovative solutions are necessary to decrease costs, while ensuring the high quality of care. A considerable part of the costs is related to hospital admission, for example for the necessary equipment and personnel. One of the solutions to decrease healthcare costs is to limit the length of hospital stay. This could be achieved by providing care at home, for example with informal care, home care and/or the use of innovative technologies. SensiStep is an innovative technology that has the potential to play an important role in the upcoming shift from hospital care to home care.

Currently, patients are unable to rehabilitate at home as supervision by experts is essential, but unavailable or extremely expensive in the home setting. In this way, the demanded high quality of care is not guaranteed. By using SensiStep at home, patients are able to train gait individually, without the physical presence of expensive healthcare professionals. Instead, healthcare professionals are able to remotely supervise training exercises and evaluate rehabilitation progress via the online Web Portal. Communication between the patient and healthcare professional could take place via the Web Portal, mobile phone or outpatient appointments. This shift from hospital care to home care is foreseen in the nearby future and SensiStep is able to play a crucial role in the field of rehabilitation at home. Currently, the development innovative rehabilitation programs with Sensistep at home is already explored (see further under 'planning and realisation'). The preliminary results are promising and this could lead to lower economic costs, while ensuring the high quality of care.

\section{Target audiences}

The main objective of this thesis was to develop an innovative rehabilitation tool to improve existing rehabilitation programs after lower extremity fractures. This resulted in the biofeedback system SensiStep that could be used by various patients following all types of lower extremity fractures. These patients are located either in the hospital, the rehabilitation clinic or at home. The results described in this thesis were gathered in the hospital and the rehabilitation clinic. The beneficial effects of monitoring and providing real-time biofeedback have been shown. The results of this thesis are not limited to lower extremity fractures, but could also be used for the rehabilitation in other diseases, such as 
stroke, diabetes or an amputation of the lower extremity. One important aspect in the design of the system was that it should be user-friendly and affordable for all types of patients.

SensiStep was developed in close collaboration between universities and SME's. The SME's ensured the commercial availability of the biofeedback system to society. Institutes, patients and other interested people are able to buy SensiStep for institutional or private use against relatively low costs (i.e. compared to other commercially available biofeedback systems).

In order to increase the usage of innovative technologies such as SensiStep, it is important to create public awareness of the availability and possibilities of these technologies. Scientific publications, congresses and expert workshops are important dissemination activities to create awareness at the level of experts, such as healthcare professionals. Other outreach activities are important to create awareness in society and potential users of SensiStep, such as patient groups. In this light, several outreach activities were already done to inform various interested people.

\section{Dissemination and outreach}

Important results were communicated to healthcare professionals in scientific publications and congresses (see Addendum: list of publications and international abstract and poster sessions). In addition, several workshops and expert courses were organised to train various expert groups with SensiStep. The most important aspects were to create awareness and familiarity with SensiStep, but also to show the possibilities of innovative techniques in medicine to experts. The expert courses were organised at three different platforms: 1) Nederlandse Vereniging voor Fysiotherapie in de Geriatrie (NVFG), 2) Nederlands Paramedisch Instituut (NPi) and 3) Sportmedisch Wetenschappelijk Jaarcongres (SMWJC). The target audience varied between experts, healthcare professionals and (para)medical students. Dissemination to these people is important as they are considered to be the main users of the product. Therefore, also a SensiStep symposium for experts and other interested people was organised at the University Medical Center Utrecht. Various presentations and workshops were organised to increase knowledge and familiarity with SensiStep.

Outreach and communication to society and other interested people was established through various channels, including popular magazines, newsletters and radio. A broad 
audience was reached via interviews in Smarthealth (online available at

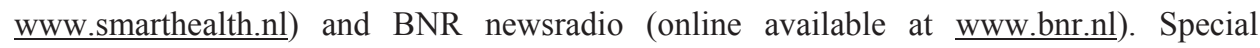
newsletters at innovation platform VitaValley (online available at www.vitavalley.nl) and geriatric rehabilitation center Zorggroep Groningen (online available at www.zorggroepgroningen.nl) created public awareness of SensiStep.

\section{Innovation}

The development of biofeedback systems has a long history, as described in the introduction of this thesis. Many improvements have been made over the past years that resulted in the development of SensiStep. Recently, many companies, including multinationals, became interested in the measurement of gait patterns in the ambulatory situation. This resulted in the development of several devices, such as smart socks, smart soles and smart shoes. The largest difference compared to SensiStep is that these products focus on sport activities, such as running, instead of measuring patients in the clinical setting. The error margins in smart devices for running are allowed to be much larger compared devices used for clinical purposes. Therefore, SensiStep used innovative sensor technologies and is currently the state-of-the-art biofeedback system in ambulatory gait analysis techniques. Further innovation is necessary to improve aesthetics and comfort, for example to increase usage at home.

\section{Planning and realisation}

Recently, Sweden was one of the first members of the European Union implementing the shift from hospital care to home care in order to reduce the high medical costs. This Swedish situation provided the optimal environment to test SensiStep in the home setting. The experimental set up was to use SensiStep under direct supervision of the physical therapist at the patients' home. The first results showed that patients improved gait if SensiStep was used during training sessions. However, these are preliminary results in an ongoing project and results need to be further evaluated in the future. This is a first step in the right direction and improvements are still possible as patients could train without direct supervision of the physical therapists. The plan is to investigate these possibilities in future research.

One of the requirements to provide care at home with SensiStep is that these rehabilitation programs will be reimbursed by insurance companies. Therefore, meetings with insurance 
companies are envisaged in the nearby future to explain the potential of SensiStep. The first reactions from insurance companies are promising. The beneficial aspects of SensiStep need to be further discussed and focussed both on the healthcare perspectives as costs effectiveness. For costs effectiveness reasons, it is important to consider the use of SensiStep not only after lower extremity fractures, but also in other diseases such as stroke and diabetes.

\section{Conclusion}

In conclusion, this thesis resulted in a new and publicly available biofeedback system: SensiStep. Dissemination and outreach activities have created public awareness of the possibilities of this biofeedback system. One important step to increase the usage of SensiStep is to arrange reimbursement for costs by insurance companies. The potential of SensiStep in rehabilitation is evident, especially with the foreseen shift from hospital care to home care in mind. The society could benefit from this system in the nearby future, not only after lower extremity fractures, but in various diseases. 


\section{List of publications}

Raaben, M., Redzwan, S., Augustine, R., \& Blokhuis, T. J. (2018). COMplex Fracture Orthopedic Rehabilitation (COMFORT) - Real-time visual biofeedback on weight bearing versus standard training methods in the treatment of proximal femur fractures in the elderly: study protocol for a multicenter randomized controlled trial. Trials, 19(1), 220.

Houben, I. B., Raaben, M., Batenburg, M. V. B., \& Blokhuis, T. J. (2018). Delay in weight bearing in surgically treated tibial shaft fractures is associated with impaired healing: a cohort analysis of 166 tibial fractures. European Journal of Orthopaedic Surgery \& Traumatology, 1-8.

Bastian, O. W., Mrozek, M. H., Raaben, M., Leenen, L. P., Koenderman, L., \& Blokhuis, T. J. (2018). Serum from the Human Fracture Hematoma Contains a Potent Inducer of Neutrophil Chemotaxis. Inflammation, 1-9.

Raaben, M., Vogely, H. C., \& Blokhuis, T. J. (2018). Real-time visual biofeedback to improve therapy compliance after total hip arthroplasty: a pilot Randomized Controlled Trial. Gait \& posture, 61, 306-310.

Raaben, M., Holtslag, H. R., Leenen, L. P., Augustine, R., \& Blokhuis, T. J. (2018). Realtime visual biofeedback during weight bearing improves therapy compliance in patients following lower extremity fractures. Gait \& posture, 59, 206-210.

Pla, A., Mordvanyuk, N., López, B., Raaben, M., Blokhuis, T. J., \& Holtslag, H. R. (2017). Bag-of-steps: Predicting lower-limb fracture rehabilitation length by weight loading analysis. Neurocomputing, 268, 109-115.

Raaben, M., Holtslag, H. R., Augustine, R., van Merkerk, R. O., Koopman, B. F., \& Blokhuis, T. J. (2017). Technical aspects and validation of a new biofeedback system for measuring lower limb loading in the dynamic situation. Sensors, 17(3), 658.

Raaben, M., \& Blokhuis, T. J. (2015). De technisch geneeskundige: een nieuwe professional in de zorg. Nederlands Tijdschrift voor Traumachirurgie, 23(5), 109-112. 


\section{International abstracts and poster sessions}

Raaben, M., Redzwan, S., Augustine, R. \& Blokhuis, T.J. (2018). Innovative measurement of rehabilitation progress in elderly with a hip fracture: a new endpoint, IEEE CAMA 2018, Sweden.

Raaben, M., Houben, I. B., Batenburg, M. V. B., \& Blokhuis, T. J. (2018). Delay in weight bearing in surgically treated tibial shaft fractures is associated with impaired healing: a cohort analysis of 166 tibial fractures, ESTROT 2018, Germany.

Raaben, M., Redzwan, S., Augustine, R. \& Blokhuis, T.J. (2018). A new technique for rehabilitation using a sensor for force measurement, EFORT 2018, Spain.

Raaben, M., Redzwan, S., Augustine, R., Granqvist, A., Derks, L., Schwietert, H.R. \& Blokhuis, T.J. (2018). COMplex Fracture Orthopedic Rehabilitation (COMFORT) Ambulatory monitoring of rehabilitation patterns after proximal femur fractures in elderly, NVvTG 2018, The Netherlands.

Raaben, M., Redzwan, S., Augustine, R. \& Blokhuis, T.J. (2018). COMplex Fracture Orthopedic Rehabilitation (COMFORT) - Monitoren van de revalidatie van ouderen met een heupfractuur en het effect van het geven van biofeedback, LTG, The Netherlands.

Raaben, M., Redzwan, S., Augustine, R., Granqvist, A., Galmin, R., Derks, L., Buijs, L., Schwietert, H.R. \& Blokhuis, T.J. (2017). COMplex Fracture Orthopedic Rehabilitation (COMFORT) - Ambulatory monitoring of rehabilitation patterns after proximal femur fractures in elderly, ESTROT 2017, United Kingdom.

Raaben, M., Redzwan, S., Augustine, R., Granqvist, A., Derks, L., Schwietert, H.R. \& Blokhuis, T.J. (2017). COMplex Fracture Orthopedic Rehabilitation (COMFORT) Ambulatory monitoring of rehabilitation patterns after proximal femur fractures in elderly, ECTES 2017, Romania.

Roelofs, D.R., Raaben, M., Schoneveld, J.S., Redzwan, S., Hofman, J., van Haren, C. \& Blokhuis, T.J. (2016). Biofeedbacktraining helpt geriatrische patiënten bij partieel belasten van de onderste extremiteit, SEOHS 2016, The Netherlands.

Mrozek, M., Bastian, O.W., Raaben, M., Koenderman, L., Leenen, L.P.H. \& Blokhuis, T.J. (2016). De chemotaxie van neutrofielen richting het fractuurhematoom kan in vitro worden 
geblokkeerd door Chemotaxis Inhibitory Protein of S. aureus (CHIPS), SEOHS 2016, The Netherlands.

Raaben, M., Wisse, L., Leenen, L.P.H., Holtslag, H.R., van Merkerk, R.O. \& Blokhuis, T.J. (2016). Monitoren van belasten in een ambulante setting: de SensiStep, Nationale Traumadagen 2016, The Netherlands.

Raaben, M., Nijenhuis, I., Augustine, R., Leenen, L.P.H., Holtslag, H.R. \& Blokhuis, T.J. (2016). Monitoring weight bearing in an ambulant setting: the SensiStep, ECTES 2016, Austria.

Raaben, M., Roelofs, D.R., Leenen, L.P.H., Holtslag, H.R., van Merkerk, R.O. \& Blokhuis, T.J. (2016). Monitoren van belasten in een ambulante setting: de SensiStep, NVT Assistentensymposium 2016, The Netherlands.

Raaben, M., Wisse, L., Leenen, L.P.H., Holtslag, H.R., van Merkerk, R.O. \& Blokhuis, T.J. (2015). Monitoren van belasten in een ambulante setting: de SensiStep, SEOHS 2015, The Netherlands.

Raaben, M., Bastian, O.W., Koenderman, L., Leenen, L.P.H. \& Blokhuis, T.J. (2014). Chemotaxis of neutrophils towards the fracture hematoma in vitro does not depend on the complement system, CXCR1 or CXCR2, Nationale Traumadagen 2014, The Netherlands. 


\section{Acknowledgements}

Mijn interesse voor het wetenschappelijk onderzoek werd aangewakkerd tijdens mijn stages en klinisch fellowship Technische Geneeskunde. Toen ik vier jaar geleden de kans kreeg om onderdeel te zijn van het internationale COMFORT project en daar mijn promotieonderzoek van te maken greep ik deze kans met beide handen aan. Het was een geweldig avontuur met telkens weer interessante uitdagingen. Ik ben dan ook ongelofelijk trots op het uiteindelijke resultaat in de vorm van dit proefschrift.

Het is ook een feit dat ik dit proefschrift nooit had kunnen schrijven zonder de steun van veel collega's, familie en vrienden. Ik wil graag iedereen bedanken die heeft bijgedragen aan het tot stand komen van dit proefschrift. In het bijzonder:

Dr. Blokhuis, beste Taco, we hebben elkaar leren kennen in 2012 en ik kan wel zeggen dat we direct een goede klik hadden. Samen zijn we begonnen aan het avontuur om te onderzoeken welke chemotactische factoren in het fractuurhematoom nou zorgen voor die gigantische influx van neutrofielen kort na de fractuur. Vervolgens zijn we opnieuw gaan pionieren om, in samenwerking met de NVvTG, het klinisch fellowship Technische Geneeskunde op te zetten. Het leidde tot een promotietraject, waarbij we veel hebben gelachen, gediscussieerd en interessante ontdekkingen hebben gedaan. Het heeft uiteindelijk geresulteerd in dit proefschrift. Ik wil je graag bedanken voor alle vertrouwen die je me hebt gegeven in de afgelopen jaren.

Prof. dr. Poeze, beste Martijn, dank voor je support tijdens dit promotietraject en dat ik bij je heb kunnen promoveren. Ik heb mijn bezoeken aan het Maastricht UMC+ en onze gesprekken over SensiStep en ander wetenschappelijk onderzoek altijd als erg fijn ervaren.

De leden van de leescommissie, bedankt voor uw bereidheid om zitting te nemen in de beoordelingscommissie van dit proefschrift.

Dr. ir. Schwietert, beste Henk, in je rol als CEO van Evalan BV hebben we elkaar vaak gesproken over SensiStep en de mogelijkheden om het systeem te verbeteren, zodat het voldoet aan de wensen van de markt. Halverwege 2015 zijn we samen begonnen aan de COMFORT studie. We hebben ook samen de enorme groei van Evalan BV meegemaakt, waarna je genoodzaakt was om sommige taken over te dragen naar nieuwe medewerkers. Erg mooi om deze groei van dichtbij mee te maken. Bedankt voor alle support in de COMFORT studie! 
Leon, jij was altijd het aanspreekpunt binnen Evalan als er dingen geregeld moesten worden. Een extra SensiStep? Regel ik! Problemen met de app? Kom maar door met het probleem, dan zorg ik voor de oplossing! Je dacht ook mee in het onderzoek en hielp met de data-analyse, waardoor we zeker vorderingen hebben gemaakt. Dit geldt ook voor de BDA: "moeten we dat apparaat niet een keer testen op een biefstuk?"

Dr. Augustine, dear Robin, we first met at the COMFORT kick-off meeting in the Ångströmlaboratoriet in Uppsala. I remember I was happy to meet you and your research group, with very kind and friendly people from all over the world. All members had a passion for research and interest in our project. I learned a lot from our discussions, both scientific and for fun. I still use your arm-wrestle techniques against my brothers...

Syaiful and the other colleagues at the Uppsala University, we had a fantastic consortium with people from all over the world. Thank you for all the meetings and interesting scientific discussions. Sometimes it was difficult due to the different fields of research, but we always managed and did interesting research that was only possible in this unique consortium of engineers and medici.

Ann, I remember us dancing around the table with Swedish snacks and drinks during Midsummer (Midsommar). Awesome! Thank you for giving me some insight in the Swedish culture. Also, thanks a lot for organising the SensiStep measurements in Sweden, together with Martin. I always enjoyed our conversations and appreciated your feedback on the research.

Dr. Holtslag, beste Herman, jij kwam jaren geleden met het idee om een schoen met drukzool te ontwikkelen om inzicht te krijgen in de belasting tijdens het revalideren. Dit idee was de basis voor de ontwikkeling van SensiStep. Ook nadat je in het AMC Amsterdam bent gaan werken hielden we contact en bleef je interesse houden in het wetenschappelijk onderzoek met SensiStep. Bedankt voor je bijdrage en input op de woensdagen!

Alle fysiotherapeuten die mij hebben geholpen met metingen in het kader van de COMFORT studie. Jullie hebben ontzettend veel werk verricht door data te verzamelen en mee te denken in het onderzoek. Mede dankzij jullie nuttige feedback over SensiStep, de COMFORT app en de BDA konden wij de studie te verbeteren. 
Alle patiënten en ook de vrijwilligers die mee wilden werken aan dit onderzoek. Ik kan me voorstellen dat er veel op je afkomt tijdens het revalideren na een fractuur. Ik heb het dan ook ontzettend gewaardeerd dat jullie instemden om metingen te verrichten ten behoeve van het wetenschappelijk onderzoek en de COMFORT studie in het bijzonder.

De studenten (technische) geneeskunde die hun bijdrage aan dit onderzoek hebben geleverd. Bedankt voor alle metingen en ondersteuning bij de data-analyse. Ook kijk ik met plezier terug op onze interessante discussies met nieuwe inzichten, die er voor hebben gezorgd dat ik ook op een andere manier naar het onderzoek ben gaan kijken.

Mijn paranimfen, Thijs en Bernard Jan, super dat jullie me willen ondersteunen in deze belangrijke en spannende dag. Eerste rondje is van mij!

Pa en ma, en mijn broertjes Thijs en Frank, bedankt voor al jullie support gedurende alle jaren. Mede dankzij al deze steun sta ik nu op dit punt om mijn doctorstitel te behalen. Thijs, nog bedankt voor de hulp bij de laatste analyses in Matlab!

Wietske, wat een jaar hebben wij achter de rug! Eerst trouwen, toen een huis kopen (of was het andersom?) en nu promoveren! Ik ben benieuwd wat er nu nog gaat komen... Ik wil je bedanken voor alle steun, het meedenken in mijn onderzoek en kritisch lezen van dit proefschrift. Ik denk dat het nu tijd is om er samen lekker van te genieten! 


\section{Curriculum Vitae}

Marco Raaben was born on 30 July 1988 in Neede, the Netherlands. After completing his primary school at de Kisveld in Neede in 2000, he went to the Atheneum at 't Assink Lyceum in Haaksbergen. He studied the Nature \& Health profile and nominally completed his exams in 2006. He continued his educational career and started the study Technical Medicine at the University of Twente in 2006. After obtaining his Bachelor of Science degree in 2010 and his Master of Science degree in 2013 he started the first fellowship Technical Medicine at the University Medical Center in Utrecht. He developed unique and specialized skills in the field of trauma and emergency surgery. During his fellowship he became interested in research and was closely involved in the development and implementation of innovative technologies used in trauma and emergency surgery. He expanded this research interest after his fellowship Technical Medicine in 2016 and started his career as $\mathrm{PhD}$ candidate at the Maastricht University, which resulted in the writing of this $\mathrm{PhD}$ thesis. 



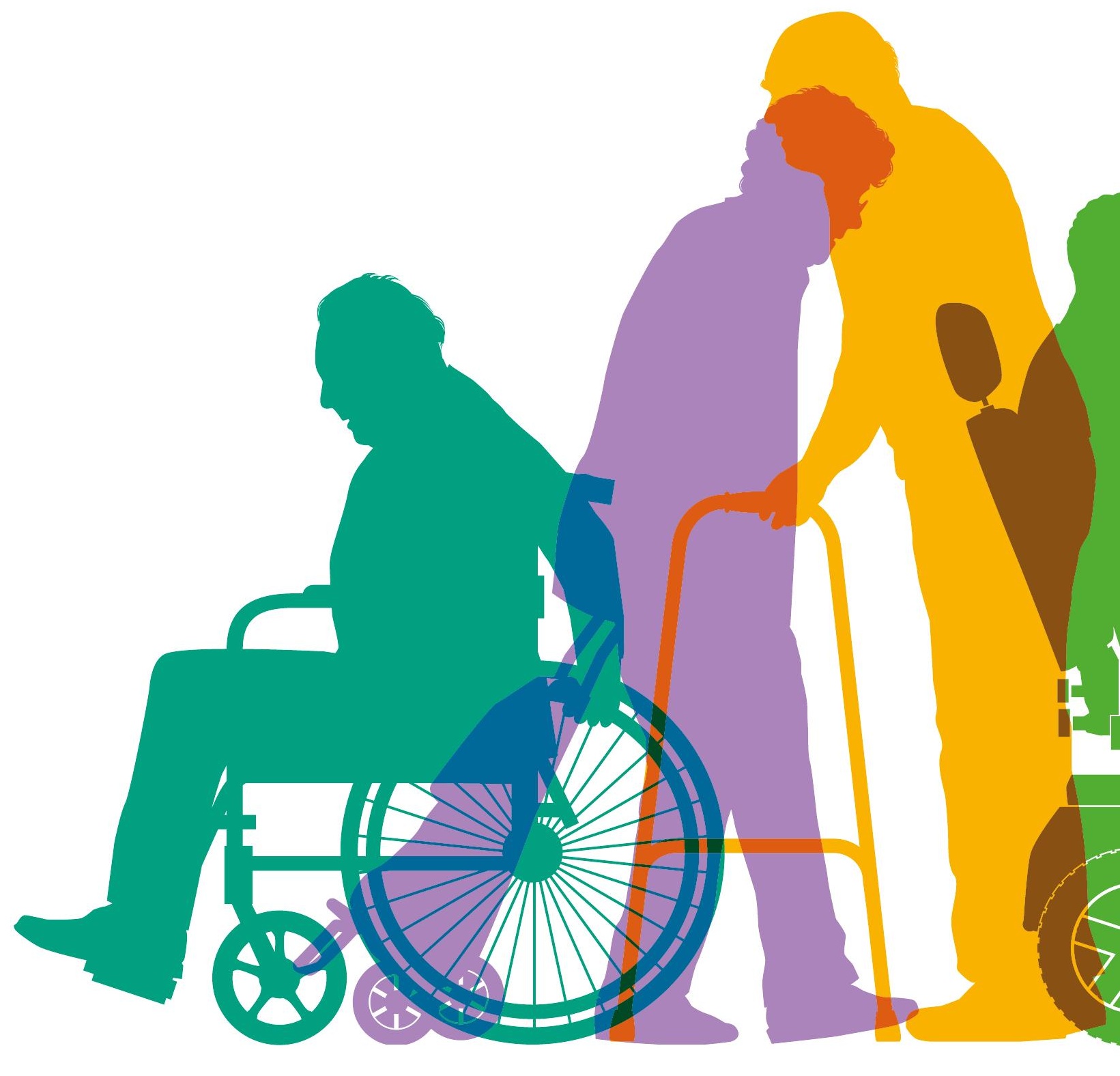

